\title{
A Polarized High-Energy Photon Beam for Production of Exotic Mesons
}

\author{
Igor Senderovich, Ph.D. \\ University of Connecticut, 2012
}

This work describes design, prototyping and testing of various components of the Jefferson Lab Hall D photon beamline. These include coherent bremsstrahlung radiators to be used in this facility for generating the photon beam, a fine resolution hodoscope for the facility's tagging spectrometer, and a photon beam position sensor for stabilizing the beam on a collimator. The principal instrumentation project was the hodoscope: its design, implementation and beam testing will be thoroughly described. Studies of the coherent bremsstrahlung radiators involved X-ray characterization of diamond crystals to identify the appropriate line of manufactured radiators and the proper techniques for thinning them to the desired specification of the beamline. The photon beam position sensor project involved completion of a designed detector and its beam test. The results of these shorter studies will also be presented. The second part of this work discusses a Monte Carlo study of a possible photo-production and decay channel in the GlueX experiment that will be housed in the Hall D facility. Specifically, the $\gamma p \rightarrow X p \rightarrow b_{1} \pi \rightarrow \omega \pi^{+} \pi^{-}$channel was studied including its Amplitude Analysis. This exercise attempted to generate a possible physics signal, complete with 
internal angular momentum states, and be able to reconstruct the signal in the detector and find the proper set of $J^{P C}$ quantum numbers through an amplitude fit. Derivation of the proper set of amplitudes in the helicity basis is described, followed by a discussion of the implementation, generation of the data sets, reconstruction techniques, the amplitude fit and results of this study. 


\title{
A Polarized High-Energy Photon Beam for Production of Exotic Mesons
}

\author{
Igor Senderovich \\ B.A., College of Arts and Sciences, Cornell University, Ithaca, NY, 2005 \\ M.S., University of Connecticut
}

\author{
A Dissertation \\ Submitted in Partial Fullfilment of the \\ Requirements for the Degree of \\ Doctor of Philosophy \\ at the \\ University of Connecticut
}

2012 
Copyright by

Igor Senderovich 


\section{APPROVAL PAGE}

Doctor of Philosophy Dissertation

\section{A Polarized High-Energy Photon Beam for Production of Exotic Mesons}

Presented by

Igor Senderovich, B.A., M.S.

Major Advisor

\section{Richard Jones}

Associate Advisor

Thomas Blum

Associate Advisor

$$
\text { Peter Schweitzer }
$$

University of Connecticut

2012 
This work is dedicated to my parents, Alla and Eugene Senderovich. Indeed they made this and all my other projects possible through their efforts and endless sacrifices to make me who I am today. 


\section{ACKNOWLEDGMENTS}

A list to acknowledge the contributions to a work like this can hardly be written in a complete and accurate manner. Neither can the language in this short foreword capture fully the value of all the gifts I received on the road of this work.

First and foremost, I owe a deep bow to my parents, from whom I received a most special upbringing and a most incredible gift of opportunities that they made possible with their struggles. Their support during my studies is immeasurable as is the support of my brother and friends, who bore our physical distance graciously throughout. My most serious debt is to my dear wife-to-be, Kinga Esther Neder, who supported me with devotion and patience despite a painfully long distance and sacrificed during already trying times for my sake.

Of course, I would not have endeavored on the path of this study nor finished it as enriched as I am without my teachers. I was inspired to go into physics thanks to my first high school physics teacher, Dr. Raymond Rapaport. Though driven to pursue this subject, I would not have been able to take on graduate study without work with and encouragement from Dr. Charles K. Sinclair, Professor David M. Lee and Professor Ivan V. Bazarov. Of course, it is my graduate adviser and mentor, Professor Richard T. Jones, who made me the physicist I am today and who, I know, has planted seeds of further growth such that I am not yet even fully aware of my debt to him.

This work is just a small addition to the efforts of the dedicated and warm people of the GlueX collaboration, who taught me much and shaped my development during our work together. The academic environment and the people of the University of Connecticut Department of Physics fostered my growth and granted me a wonderful chapter of my life. 


\section{TABLE OF CONTENTS}

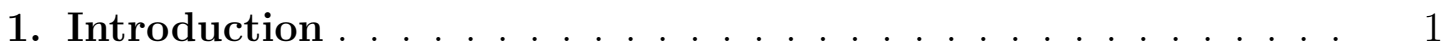

1.1 Introduction . . . . . . . . . . . . . . . . . . 1

1.2 Meson Spectroscopy . . . . . . . . . . . . . . . . . . . . . . 2

1.2.1 The Constituent Quark Model . . . . . . . . . . . . . . . . 2

1.2.2 Beyond the Quark Model . . . . . . . . . . . . . . . . . 4

1.2.3 The Experimental Situation . . . . . . . . . . . . 6

1.3 Introduction to the GlueX Experiment $\ldots \ldots \ldots \ldots \ldots$

1.3 .1 Tracking . . . . . . . . . . . . . . . . . . . . 10

1.3 .2 Calorimetry . . . . . . . . . . . . . . . . . . . . 12

1.3.3 Particle Identification $\ldots \ldots \ldots \ldots$

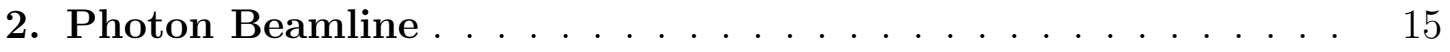

2.1 Photon Beam . . . . . . . . . . . . . . . . . . . . 15

2.1 .1 Collimation . . . . . . . . . . . . . . . . . . . 19

2.1 .2 Acitve Collimator . . . . . . . . . . . . . . . 22

2.1 .3 Diamond Characterization . . . . . . . . . . . . . . . . . 28

3. Tagger Microscope . . . . . . . . . . . . . . . . . . . 37

3.1 Introduction . . . . . . . . . . . . . . . . 37

3.2 Silicon Photomultipliers _. . . . . . . . . . . . . . . . . . 44

3.3 Amplifier Electronics . . . . . . . . . . . . . . . . . 50

3.3 .1 Requirements . . . . . . . . . . . . . . . . . 50

3.3 .2 Individual and Summed Readout . . . . . . . . . . . . . 51

3.3 .3 Gain . . . . . . . . . . . . . . . . . . . 52

3.3.4 Frequency Response and Bandwidth . . . . . . . . . . . . 53 
3.4 Control Electronics . . . . . . . . . . . . . . . . . 57

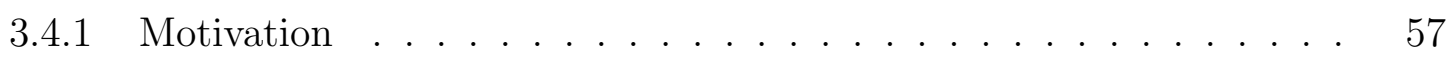

3.4.2 Implementation . . . . . . . . . . . . . . . . . 59

3.4.3 Implementation details of the FPGA . . . . . . . . . . . . . 62

3.4.4 Communication Cycle ................. 63

3.5 Beam Test . . . . . . . . . . . . . . . . . 67

4. Analysis ......................... . . . 71

4.1 Introduction . . . . . . . . . . . . . . . 71

4.2 Angular Amplitudes: General Relations . . . . . . . . . . . . 73

4.2.1 Angular Distribution of a Two-Body Decay . . . . . . . . . . 73

4.2.2 Isospin Projections . . . . . . . . . . . . . . . . 75

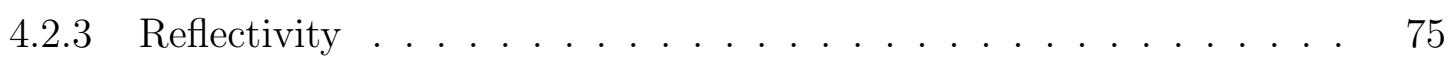

4.3 Application ........................ 76

4.3.1 Photon-Reggeon-Resonance vertex . . . . . . . . . . . . 77

4.3.2 Decay of t-channel resonance $X \ldots \ldots \ldots$. . . . . . . 79

4.3.3 Assembly of the full amplitude . . . . . . . . . . . . . 81

4.3.4 Proton states and individual decay amplitudes . . . . . . . . . 83

4.3.5 Mass dependence . . . . . . . . . . . . . . . 84

4.3.6 Describing $s$ and $t$ dependence ............. 86

4.3.7 Summing over photon polarization . . . . . . . . . . 86

4.4 Implementation . . . . . . . . . . . . . . . 87

4.4.1 Amplitude Parameters . . . . . . . . . . . . . . . 88

4.4.2 Monte Carlo check of amplitude orthogonality . . . . . . . . . . 89

4.5 Monte Carlo Event Generation _. . . . . . . . . . . . . . . . 91

4.5.1 Event Generators . . . . . . . . . . . . . . . 91 
4.5.2 Generating Events: the Computing Challenge . . . . . . . . . . . 93

4.6 Signal Reconstruction and Analysis . . . . . . . . . . . . . 97

$4.6 .1 \pi^{0}$ Reconstruction . . . . . . . . . . . . . . . . 98

4.6.2 Charged Track Reconstruction and Identification . . . . . . . . 101

4.6.3 Event Rendition Selection and Kinematic Fitting . . . . . . . . 102

4.6.4 Suppression of Contamination from $\Delta$ resonances . . . . . . . . . 104

4.6.5 Cut Threshold Optimization . . . . . . . . . . . . . . 107

4.7 Fitting (Simulated) Data: Maximum Likelihood Method . . . . . . 108

4.8 Fit Results . . . . . . . . . . . . . . . . . . . . . . . . 110

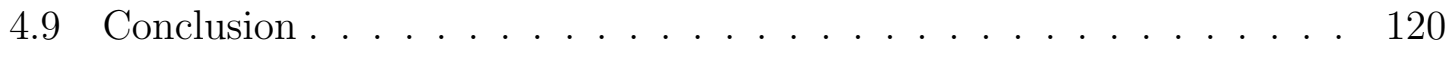

$\begin{array}{ll}\text { Bibliography } & 123\end{array}$ 


\section{LIST OF FIGURES}

1.1 Plan of the $12 \mathrm{GeV}$ upgrade. The electron gun is represented by the green cube. A bunch makes only one pass through any one arc. .

1.2 Schematic cut-away view of the GlueX detector. Not visible is the time of flight system: a thin wall of scintillating counters stands in front of the FCAL with Start Counter instrumented with scintillators around the target. Barrel Calorimeter's fast readout contributes timing information at large angles. This forms the baseline design of the detector, with further PID detector systems under consider-

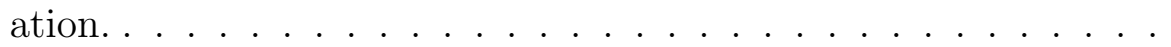

1.3 These plots show the distributions of simulated $\mathrm{dE} / \mathrm{dx}$ measurements for pions, kaons and protons launched at $90^{\circ}$ (left) and $15^{\circ}$ (right) from the target. Good separation between these particles appears possible at moderate momenta, especially at higher angles, where more straws are traversed. . . . . . . . . . . . . .

1.4 This figure shows the scheme of forward drift chamber wiring. Note the alternation of field and sense wires between the cathode strips to create drift cells for ionization timing. The shown relative angular offset of the cathode strips and the $60^{\circ}$ shifts of similar assemblies in the construction of an FDC package help disambiguate the FDC crossing points. . . . . . . . . . . . . . . . 
1.5 The scintillator and corrogated lead sheet layering pattern for the barrel calorimeter is shown on the left. The expected time of flight measurement uncertainties as a function of momentum for different charged particle species is shown on the right. . . . . . . .

1.6 This plot shows the expected TOF wall resolution of pions, kaons and protons, assuming $3 \%$ momentum uncertainty, a path length uncertainty of $1 \mathrm{~cm}$, and time resolution from the detector and readout of $80 \mathrm{ps}$ and $40 \mathrm{ps}$, respectively. . . . . . . . .

2.1 Tagger hall instrumentation is illustrated with a horizontal section along the beam axis and the symmetry plane of the tagger dipole

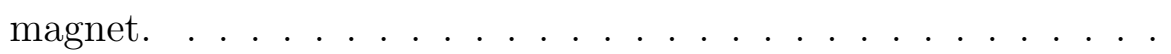

2.2 Degree of linear polarization of the coherent bremsstrahlung photon beam as a function of energy, under varying collimation conditions. The dashed lines show the trajectory of the maximum polarization fraction as a function of the peak energy. These are shown for three collimator aperture scenarios. . . . . . . . . . . . .

2.3 Distribution of bremsstrahlung photons in polar angle and energy, with the coherent part shown in red bands. . . . . . . . . . . . . 20

2.4 Simulated photon spectra with various degrees of collimation. . . . . 21

2.5 Exploded view of the Active Collimator assembly. In this view the beam entry is from the top. From top to bottom, the layers are: face plate, boron-nitride insulating cup, tungsten "pin-cushions", grounded cup. The top and bottom pieces form a grounded Faraday cup to shield the detector. . . . . . . . . . . . . . . . 
2.6 Plot of the five highest gain settings of the Advanced Research Instruments PMT-5R amplifier, labeled by their nominal gain settings. The vertical scale shows higher values than advertised and may suffer from a systematic normalization error. The relative bandwidths of the gain modes is the most important result. . . . . . . . . 25

2.7 DC current from the outer (left) and inner (right) tungesten wedges as a function of the Active Collimator's displacement with respect to the beam. The apparent cross-talk has been understood as a symptom of an improper connection, which should not appear in the final system. . . . . . . . . . . . . . . .

2.8 The DC current asymmetry defined as the difference of the sum of the two inner tungsten plates as a function of beam position. . . . . .

2.9 The Fourier transform averaged over $1001 \mathrm{~s}$ traces from inner tungsten pieces taken at incremental positions from $-2.5 \mathrm{~mm}$ to $+2.5 \mathrm{~mm}$ from the beam center. The bandwidth corresponds well to that measured on the bench for the $10^{10}$ gain mode. . . . . . . . . .

2.10 Top row: Plots of local rocking curve width for an electron grade wafer in the vertical and horizontal scan orientations. Bottom row: Corresponding plots of the centroid of the local rocking curves. This $300 \mu \mathrm{m}$-thick, virgin sample clearly holds its normal to the scattering plane uniformly across the surface. . . . . . . . . . 
2.11 Top row: Plot of local rocking curve width for the non-electron grade "plate" wafer, shown for the vertical and horizontal orientation scans. Note how the inherent rocking curves for most of the diamond surface are comparable to those of the expensive electrongrade diamonds. Bottom row: Plot of the centroid of the local rocking curves examined in the vertical and horizontal scans. This thick, virgin sample clearly holds its normal to the scattering plane uniformly across its surface. . . . . . . . . . . . .

2.12 Top row: Plots of local rocking curve width in the two scan orientations for an electron-grade CVD crystal thinned by SINMAT to $9 \mu \mathrm{r}$ using a combination of RCMP and reactive ion etching. Notice that except for spots of significant structural damage, the local crystal mosaic spread is still small. The corresponding distributions of the centroid of the local rocking curves from these scans is shown in the bottom row. They demonstrate that the thinned diamond now flexes well beyond the beamline design tolerance. . . . . . . . . 35

2.13 Top row: Plot of the local rocking curve widths for the $10 \mu \mathrm{m}$ Element6-thinned sample, from scans in the two orientations. The wafer appears to have a small local mosaic spread over most of the surface, with some spots exceeding specification. Bottom row: Corresponding plots of the centroid of the local rocking curves. It is clear that the thin wafer does not hold its form: the normal to the scattering plane travels across the diamond surface and exceeds the design specification. . . . . . . . . . . . . . . . . 
3.1 Raw rates on the tagger hodoscopes as a function of photon energy are shown by the upper (black) curve. The post-collimator rate is shown below (red) with the segment counted by the fixed array and microscope highlighted in yellow (light shade) and green (dark shade), respectively. . . . . . . . . . . . . . . . . . . 38

3.2 The distribution of electron flux transverse to the dispersed electron beam is shown. The fraction of electrons producing a photon that clears the collimator is highlighted. This figure makes a case for improving tagging efficiency by restricting the tagger acceptance band in the y-direction. . . . . . . . . . . . .

3.3 This is how the focal plane instrumented with the scintillating fibers looks to the incoming electrons. The red (dark) stripe shows the beam intensity profile once it is centered on a row of fibers. Thin, light gray lines show the segmentation of individual fibers; thick black lines indicate boundaries between fiber bundles. In this segmentation plan a bundle has 25 fibers, which can be read-out by a single 30-channel SiPM amplifier board. Note that other segmentation plans are possible; in all cases, the electronics allows for individual fiber readout in the edge column of any bundle. . . . .

3.4 A conceptual design of the focal plane scintillating fiber counter instrumentation (not to scale). Waveguides coupling scintillators to the readout sensors are not shown in the top view but their mating end is shown in the detail sketch in the lower-left. . . . . . . . . 
3.5 This diagram shows the signal flow sequence, starting from the electron's scintillation in the fiber all the way to readout. The bundles of 5 channels here represent a column of scintillating fibers on the focal plane (one energy bin). Hence, these signals are summed during normal tagger operation. However one sum group per electronics board will also have its individual signals patched out for readout to offer a two-dimensional profile of the dispersed electron beam to help center the beam on a single fiber row. About 5 such columns out of 100 will be read out in this fashion. . . . . . . .

3.6 A multi-Poisson model fit to a sample spectrum from a series of pulses collected from Photonique SSPM-06x SiPM illuminated by small flashes of light from and LED. . . . . . . . . . . . . .

3.7 Sensitivity of SiPM photo-detection efficiency, gain and dark rate to the bias voltage and temperature. . . . . . . . . . .

3.8 Schematic of the SiPM bias, amplifier and summing circuit. A functional diagram of this electronics is given above the schematic, roughly illustrating the function of the transistor stages. Note that five instances of the circuit to the left of the vertical dashed line (representing the initial pre-amplifier and individual channel buffer) with its SiPM connect to a single summing circuit on the right of the dashed line. For simplicity, just one pre-amplifier is drawn connected to the summer. . . . . . . . . . . . . 55 
3.9 The plots show the simulated amplifier response in frequency and time domains. An approximation to the input signal shape and spectrum is given based on some un-amplified recorded signals from the SiPM deconvolved based on expected oscilloscope and cable bandwidth

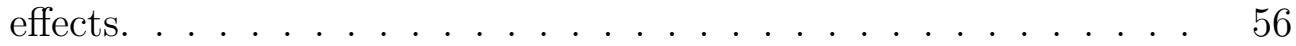

3.10 The design frequency response is compared to the measured in the first manufactured prototype. The time domain pulses had a longer fall time, corresponding with the low frequency enhancement and showed significant ringing compatible with the large resonance near

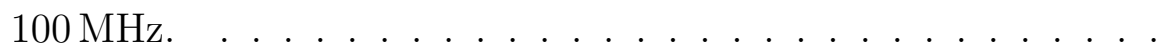

3.11 This diagram illustrates the communication scheme between the control board components and some internal FPGA modules. Specifically, it shows how communication on the internal FPGA "transceiver control bus" is orchestrated with a state register. The register codes serve as "chip enable" bits to prevent multiple drivers from writing on the bus. Modules pass control to each other by setting this code after they are done to create the proper chain of events to enable Ethernet communication with the master-computer. . . . . . . .

3.12 This data flow diagram shows the procedure for initializing the detector electronics and the transition to the normal operating regime. Note how this process does not presuppose any records of the MAC or geographic addressed of electronics bundles. The control board reports its MAC address and its geographic address (encoded by its physical slot) allowing the computer to build a lookup table. The system then switches to unicast for further communication. . 
3.13 Format of Ethernet packets sent to the detector. Left: General format of Ethernet packets used to communicate with the Tagger Microscope. Right: Layout of the "program packet" data for SiPM bias voltage specification. Note that the channel numbering here represents DAC's channel numbers which map to actual SiPM channels depending on the trace-routing convenience on the electronics boards. 67

3.14 Difference between the "accelerator RF" clock and the timing from pulse analysis is shown for a higher (left) and lower (right) effective thresholds with respect to the signal amplitude. The time resolution is clearly worse for the higher threshold. Overlap between pulse timing distributions indicates the rate of accelerator bunch misidentification. . . . . . . . . . . . . 70

3.15 Measured time resolution is shown as a function of the effective threshold used in analysis. Extrapolation to the designed threshold around $30 \%$ suggests that the 200 ps requirement can be met. . . . . . .

4.1 Feynman diagram for photo-production of a resonance $X$ in recoil from a proton, with the decay of the resonance to $b_{1} \pi$. Only the dominant modes of $b_{1}$ and $\omega$ decays are included into the overall process amplitude. The inverse isospin combination to this diagram, $b_{1}^{-} \pi^{+}$, is included. . . . . . . . . . . . . . . .

4.2 Distribution of reconstructed invariant mass of $2 \gamma$ in a Pythia event sample. The left plot was produced using the base calorimetry algorithms and the current model of BCAL noise. The right plot was produced without BCAL noise hits and with tweaks to shower association and photon hypothesis identification algorithms. . . . 100 
4.3 Left: Distributions of the difference between the measured time of flight and that expected assuming speed of light. The distributions are taken from reconstruction without any analysis filters. (Note the enhancement in the $\pi^{+}$distribution due to misidentification of protons.) Right: The mass distribution that results from a $\pm 8 \mathrm{~ns}$ photon timing cut (black solid line) is compared to the raw reconstructed distribution (blue/dash). . . . . . . . . . . . .

4.4 Distributions of energy loss rates versus particle momentum magnitude for protons and $\pi^{+} \ldots \ldots \ldots \ldots \ldots \ldots$

4.5 Distributions of reconstructed normalized particle velocity $(\beta)$ versus energy for protons and pions. The expected dependence of velocity on energy is shown in dashed line. . . . . . . . . . . . . 103

4.6 Left: Reconstructed $\Delta$ invariant mass in known events with $\Delta$ resonances. Right: $p \pi$ system invariant mass in $b_{1} \pi$ events selected for minimal mass. It is clear that there is no forward-backward separation that allows any convenient cut. . . . . . . . . . 105

4.7 The upper row of plots shows the angular distributions of the decay of $\Delta$ resonances in the Pythia dataset as well as the reconstructed angular distribution. Below are the distributions of $p \pi$ system in the $b_{1} \pi$ signal data. (The event reconstruction software identifies the lowest mass $p \pi$ system as a candidate $\Delta$ and forms these quantities.) The uniform distribution of real $\Delta$ decays compared to the peaked distributions of false $\Delta$ systems offers a chance at cuts to improve signal purity. . . . . . . . . . . . . . . 106 
4.8 The Pareto-optimal front of solutions optimizing efficiency and purity of a data sample given the available cut parameters. The units are not meaningful in themselves as they scale from rates and efficiencies of a baseline set of cuts. . . . . . . . . . . . . . 108

4.9 Generated $b_{1} \pi$ invariant mass distribution using a mixture of $1^{--}$and $2^{+-}$waves. . . . . . . . . . . . . . . . .

4.10 Top: Results of a mass-independent fit of a $b_{1} \pi$ signal sample, assuming a detector of perfect acceptance and resolution. Negligible leakage (dark curves at the bottom) to false waves is seen. Bottom: The phase difference between the two coherent waves is shown, with the expected values computed in two ways: "naive" phase difference between two Breit Wigners without breakup momentum in the resonance's two-body decay and a scatter of phase differences for each thrown event. . . . . . . . . . . .

4.11 Top-left: Results of a mass-independent fit with $b_{1} \pi$ signal events passing GlueX reconstruction. Leakage mainly from the $1^{--}$to the uniform wave is apparent. Same plot with expected values, rescaled to account for this leakage, is shown in the top-right. Bottomleft: The phase difference between the two coherent waves is shown with error bars included as indicators of fit uncertainty. Accuracy of fit convergence and perhaps other systematic contributions to the error bars are not fully understood. The expected values are computed in two ways: "naive" phase difference between two Breit Wigners without breakup momentum in the resonance's two-body decay and a scatter of phase differences for each thrown event. . . 115 
4.12 Top: Results of a mass-independent fit with the events passing GlueX reconstruction. The generated $b_{1} \pi$ signal with a $40 \mathrm{nb}$ exotic $2^{+-}$ and the 120 nb photo-production physics samples are included. Some leakage of Pythia's $\omega \pi \pi$ signal into the exotic $2^{+-}$and other waves is apparent. Greater leakage at higher invariant mass is also visible, likely due to lower acceptance MC statistics in that limit. Bottom: The phase difference between the two coherent waves is shown with error bars included as indicators of fit uncertainty. Accuracy of fit convergence and perhaps other systematic contributions to the error bars are not fully understood. The expected values are computed in two ways: "naive" phase difference between two Breit Wigners without breakup momentum in the resonance's two-body decay and a scatter of phase differences for each thrown event. . . 118

4.13 The top plot shows the same results as in Figure 4.12 on the log scale for a clearer view of the leakage to the false waves. The bottom plot shows the results of a fit to the $\omega \pi \pi$ Pythia channel alone. The decays in this channel are modeled with uniform angular distributions and should, therefore, be absorbed by the uniform wave. Note the leakage to other waves, including the exotic $2^{+-}$. This may be due to the artificial structures in the angular distributions that accumulate from instances of incorrect event reconstruction. . 119 


\section{LIST OF TABLES}

3.1 Jefferson Lab's VME64x Flash ADC Module (fADC250) specifications constraining SiPM amplifier design. . . . . . . . . . . . . 50

3.2 Detailing of the performance requirements for the SiPM pre-amplifiers and how they may be translated into design features or constraints. More general additional requirements include: 1. summation of channels in groups of five with individual channel readout available on select columns, 2. minimum power consumption within the constraints of the specifications below. . . . . . . . . .

3.3 Estimate for the necessary transimpedance gain of the SiPM preamplifier at the start of the circuit's design. This estimate models the input and output signals as triangles without change of shape due to bandwidth limitations. . . . . . . . . . . . . . . 53

3.4 Allocation of the ADC channels for monitoring critical sytem voltages. 61

4.1 Diagonal and off-diagonal inner products of amplitudes. Note how the latter set ("external quantum numbers") have off-diagonal inner product on the order of $10^{-9}$. This likely indicates term by term cancellation in the integral. By comparison, the "internal quantum numbers" (those summed over) do not cancel term by term and approach zero as a function of the accumulated statistics. . . . .

4.2 Input waves for the decay $X \rightarrow b_{1} \pi \ldots \ldots \ldots 111$ 


\section{Chapter 1}

\section{Introduction}

\section{$1.1 \quad$ Introduction}

Hadrons, like the familiar protons, neutrons and pions, have long been known to be composite objects [8]. Early elastic scattering experiments measured form-factors of the proton that are inconsistent with a point-like object. Subsequent inelastic scattering experiments with high momentum transfer have shown a scaling relation that resembles a collection of free sub-particles, then labeled as partons [6]. The Strong Interaction that governs the binding of these particles has been understood as being asymptotically free, or becoming weak in the short distance scale (high momentum) limit, and becoming very strong at the long distance scale. In the latter limit, the constituents no longer appear free, but rather seem to become confined. Indeed, subsequent experiments failed to isolate the constituents of hadrons.

Eventually, a theory of this interaction called Quantum Chromodynamics (QCD) emerged, describing these partons, now called "quarks", as being bound by a non-Abelian (self-interacting) gauge field [26]. The theory proposed that the coupling constant of this field "runs" with the momentum scale, explaining the weakening of this force at short distances. In this limit, the small coupling constant

allows for theoretical calculations based on perturbation theory. The predictions 
based on these calculations have been confirmed by experiment, leading to a wide acceptance of QCD. On the scale of hadrons, however, the coupling constant is too large for perturbative techniques, leading to an enduring gap between experimental data and their interpretation within the theory. To remedy this situation, a host of QCD-inspired models have been proposed for understanding hadronic states. Simultaneously, monumental efforts in numerical calculations have been made in recent years to extend theoretical predictions into the region that is beyond analytical techniques. This involves QCD calculations on a discretized Euclidean space-time lattice, referred to as lattice QCD. These theoretical efforts and the corresponding experimental work in hadron spectroscopy have been converging in recent years. This document presents an instance of experimental work in "light-meson spectroscopy" with the GlueX experiment and describes my particular contribution to this work. The following sections will introduce the models of hadronic states in greater detail to provide more of the theoretical context for the experimental work described in the later chapters.

\subsection{Meson Spectroscopy}

\subsubsection{The Constituent Quark Model}

The first successful model for classifying the large number of particles being discovered from the 1950s onward was the Quark Model. The model envisioned fermionic ( $\operatorname{spin} \frac{1}{2}$ ) hadron constituents called "quarks" $(q)$ and their anti-matter counterparts - "anti-quarks" $(\bar{q})$. It was proposed that quarks come in different flavors of two categories: those with the value of electric charge of $+\frac{2}{3}$ and those with $-\frac{1}{3}[26]$. At first, these flavors included only the up $(u)$ and down (d) quarks. With the discovery of heavy and long-lived hadrons, this list grew to 
include the strange $(s)$, charm $(c)$, bottom $(b)$ and top $(t)$ quarks. In an analogy to electric charge, which can be positive or negative, the model proposed three forms of charge for the quarks labeled with colors: red, green and blue, with their negative counterparts: anti-red (cyan), anti-green (magenta), and antiblue (yellow). ${ }^{1}$ Isolation of the Strong Interaction to the nuclear scale implied that hadrons are built up to be color-neutral objects (color-singlets). One way to achieve these is through three-quark groups called baryons, with color cancellation of the form red-green-blue. The other possibility is quark-anti-quark pairs called mesons with color-anti-color cancellation. Eventually, QCD proposed that these formed fermion currents with exchange of field quanta called "gluons" self-coupling vector bosons carrying pairs of color charge. Naturally, the field theory also included fermion loops - sea quarks. As an extension, the "Constituent Quark Model" envisions the originally-proposed quark pairings as valence quarks in a complex system of quarks and gluons, each valence quark being "dressed" by the field and carrying a larger effective mass than the current mass [14]. The constituent quarks can be broadly classified into light $(u, d, s)$ and heavy $(c, t, b)$ flavors, which results in different mass scales for mesons. This work focuses on the light-meson spectrum. The three available light quarks and three anti-quarks lead to meson groupings called "nonets" with internal classification according to flavor (i.e. isospin and strangeness) and spin quantum numbers.

The model includes a scheme of angular momentum coupling, in which a meson's quarks contribute a spin $(\vec{S})$ of 0 or 1 to the orbital angular momentum $(\vec{L})$ to form a total angular momentum $(\vec{J})$. The parity is given by $P=(-1)^{L+1}$, with the intrinsic opposite parity between a fermion and the anti-fermion contributing

\footnotetext{
${ }^{1}$ Indeed, Quantum Chromodynamics acquires its name from being a field theory of these color charges.
} 
an extra minus sign to the angular wave function parity. Similar arguments for charge conjugation lead to $C=(-1)^{L+S}$. Meson states of various such quantum numbers, including quark excitations are thought to generate the observed spectrum of resonances.

Working through all combinations of angular momentum states, it can be shown that some $J^{P C}$ combinations are not allowed: $0^{--}, 0^{+-}, 1^{-+}, 2^{+-}, 3^{-+}, \ldots$ Discovery of states with these exotic quantum numbers would present unambiguous evidence of new degrees of freedom in a meson. It should be emphasized, however, that these quantum numbers are sufficient to demonstrate new degrees of freedom, but are not necessary for non- $q \bar{q}$ excitations to exist in a meson.

\subsubsection{Beyond the Quark Model}

The self-coupling nature of the gluon field suggests a richer spectrum than what is described by the quark model. The gluonic field between the constituent quarks is understood to condense along the axis between the quarks into a body modeled as a flux-tube. Calculations have shown that this body may undergo its own excitations [23]. Hybrid mesons are thought to result from a mixture of quark and gluonic excitations. The flux tube model predicts a set of eight nonets around $1.9 \mathrm{GeV}[22]$.

Other models also suggested contribution of excitations from the glue. The Bag model allows a calculation of gluonic field modes in a cavity, resulting in transverse-electric (TE) $1_{g}^{+-}$and transverse-magnetic (TM) $1_{g}^{--}$modes with four degenerate meson states for each [15]. Each results in four meson nonets degenerate in mass. The mass of the lighter TE combination for the exotic $1^{-+}$is calculated to be $1.2-1.4 \mathrm{GeV}[4]$ [9]. 
The QCD Coulomb-gauge Hamiltonian constructs Fock states for hadrons including quark and gluon operators [30]. This approach predicts four light nonexotic hybrid nonets and three heavier exotic ones, with the lightest exotic $\left(1^{-+}\right)$ around $2.1-2.3 \mathrm{GeV}$ [12] [13].

Calculations using the QCD spectral sum rules predict an exotic hybrid in the range of $1.6-2.1 \mathrm{GeV}$ and favor the $\pi_{1}(1600)$ [33]. A later examination of these predictions compared to those of lattice QCD (to be discussed later) concluded that $\pi_{1}(1400)$ and $\pi_{1}(1600)$ may be 4 -quark states while $\pi_{1}(2015)$ may be a hybrid.

Perhaps the most compelling predictions for hybrid mesons come directly from QCD in the form of lattice calculations. Recent work in this field has given support to the proposals of the model predictions [17] [18] [16]. The work presented in these references is particularly interesting for its use of unquenched calculations: quark loops are included in the simulations. However, calculations with physical quark masses are still not possible. Instead, a series of simulations are done with regimes that yield different pion masses to as low as $\sim 400 \mathrm{MeV}$. The results are then extrapolated to the realistic mass of $\sim 140 \mathrm{MeV}$. An extrapolation is also performed in the lattice spacing to estimate the physics in the continuum. It is difficult to extrapolate to the physical pion mass with good control of the systematic errors introduced, but it is safe to say that the conjectures of exotic hybrid $1^{-+}$in the range of $1.6-2.1 \mathrm{GeV}$ are supported [30]. The calculations also predict heavier exotics $0^{+-}$and $2^{+-}$as well as some non-exotic hybrids $1^{--}$and $2^{-+}$. 


\subsubsection{The Experimental Situation}

Before reviewing the experimental results on exotic mesons, it is worth introducing the experimental technique for analyzing a measured resonance and identifying the $J^{P C}$ quantum numbers. The simple way to look at a possible resonance in particle physics is to check for an enhancement in the plot of the invariant mass of the decay system, i.e. of the sum of the presumed daughters' 4-vectors. However, to check for the characteristic phase shift and analyze the contributing quantum (scattering) states, one needs to construct a model for the observed intensity from quantum amplitudes (i.e. partial waves). The relative strengths of these amplitudes are floating complex number parameters in a fit of the model to the data. Performing a fit in each mass bin allows the analyst to track the relative phases of contributing amplitudes across the span of the resonance. Another perspective is that the invariant mass histogram is just one possible projection of the event set; a hermetic detector can record angular correlations of decay products, which can be used to interpret the symmetries and spin of the parent resonance. A sample procedure for constructing an amplitude model and a thorough description of such analysis will be presented in Chapter 4 .

There is a complicated history of experimental measurements that, at the very least, suggest exotic states in the region of $1.4-2.1 \mathrm{GeV}$. A thorough overview of these is beyond the scope of this work. I limit the discussion here to the more firm and commonly reproduced results and those relevant to the studies reported in this thesis. GAMS, VES, KEK, Crystal Barrel, and Brookhaven E852 experiments have seen hints of resonant motion in the exotic $1^{-+}$wave around $1.4 \mathrm{GeV}$ with some controversy about its interpretation. Less problematic sightings of $1^{-+}$have been made near $1.6 \mathrm{GeV}$ by VES, E852 and COMPASS 
using pion beams. VES reported its result from the combined analysis of $\eta^{\prime} \pi^{-}$, $f_{1} \pi^{-}$, and $\rho^{0} \pi^{-}$with mass of $1.62 \pm 0.02 \mathrm{GeV}$ and width of $0.24 \pm 0.05 \mathrm{GeV}$ [20]. However, the conclusions of the analysis may be significantly model-dependent and the amplitudes may not have been properly constrained [36] [30]. The analysis of E852 found a possible resonance decaying to $\rho^{0} \pi^{-}$and $f_{2}(1270) \pi^{-}$with mass of

$1.593 \pm 0.08_{-0.047}^{+0.029} \mathrm{GeV}$ and width of $0.168 \pm 0.02_{-0.012}^{+0.150} \mathrm{GeV}[2][10]$. However, a reanalysis of the $\rho^{0} \pi$ final state with higher statistics and careful study of different partial wave sets with attention to leakage into the dominant $\pi_{2}(1670)$ showed that the enhancement vanishes but the phase motion persists. In general, the E852 analysis suffers from the assumption of a flat background phase with purely resonant enhancements [30]. More recently, the COMPASS experiment observed the $\pi_{1}(1600)$ in $3 \pi$ decay with a mass of $1.660 \pm 0.010_{-0.064}^{+0} \mathrm{GeV}$ and width of $0.269 \pm 0.021_{-0.064}^{+0.042} \mathrm{GeV}[3]$. The results are reported to have been strengthened with a more recent, high statistics experimental run with the updated resonance parameters not released as of the time of this writing [21]. The E852 collaboration has also reported a $\pi_{1}(2015)$ state in the $f_{1} \pi$ and $b_{1} \pi$ decay channels with mass of $2.001 \pm 0.030 \pm 0.092 \mathrm{GeV}$ and width of $0.333 \pm 0.052 \pm 0.049 \mathrm{GeV}[28]$ [29]

\subsection{Introduction to the GlueX Experiment}

A novel Gluonic Excitation Experiment or GlueX is under construction to conduct a high statistics search for exotic hybrid mesons in photo-production on a proton (liquid hydrogen) target. The experiment is assembled in the new Hall D facility of Jefferson lab, which is a fourth fixed target experimental station served by the CEBAF continuous wave $e^{-}$beam. Construction of this facility is part of the $12 \mathrm{GeV}$ upgrade, shown in Figure 1.1: the maximum possible beam energy to the 
existing Halls A, B and C is increased from 6 to $11 \mathrm{GeV}$ and a $12 \mathrm{GeV}$ beam can be delivered to Hall D.

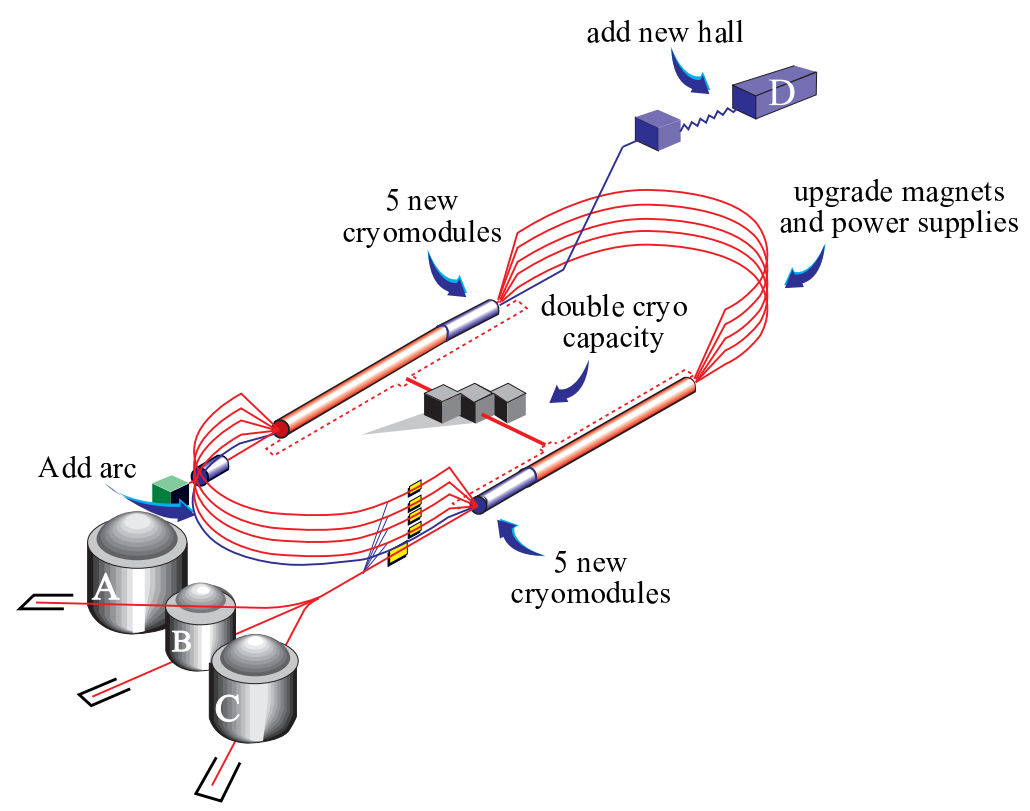

Fig. 1.1: Plan of the $12 \mathrm{GeV}$ upgrade. The electron gun is represented by the green cube. A bunch makes only one pass through any one arc.

Hall D takes advantage of the high bunch repetition rate and small emittance of the CEBAF beam to create a high rate, tagged, polarized photon beam through coherent bremsstrahlung. ${ }^{2}$ Polarization is a very important feature for GlueX physics, since states of linear polarization are eigenstates of parity. Setting up an initial state of good parity maximizes the information that can be extracted from the decay angular correlations. As will be described in Chapter 2, the facility is designed from the ground up to optimize the beam's polarization fraction, tagging efficiency and background.

The GlueX detector (Figure 1.2) is designed for high-statistics hadron spectroscopy and as close to hermetic coverage as possible to aid amplitude analysis.

\footnotetext{
${ }^{2}$ Photon running is the exclusive mode of operation for the Hall D facility: beam dump and shielding design prohibit use of electron beam in the Hall.
} 
The need for high statistics in this experiment demands an efficient data acquisition system. To this end, the detector will be instrumented entirely with fully-pipelined digitizing electronics. This means that the detector continues to acquire data as trigger decisions are formed, shifting the data into buffers at least $3.8 \mu \mathrm{s}$ deep. The trigger system selects events to keep within $3 \mu \mathrm{s}$ saving the corresponding data from the pipeline. The experiment intends to collect data with a minimum-bias trigger. A simple trigger algorithm will look for track multiplicity and minimum energy deposited to reject trivial or low energy events. ${ }^{3}$

The primary target for GlueX physics is a volume of liquid hydrogen. The cylindrical cell will be approximately $30 \mathrm{~cm}$ in length along the beamline and about $3 \mathrm{~cm}$ in diameter. The target is surrounded by 30 thin scintillator strips oriented along the beamline to instrument the Start Counter. This detector system provides the first good timing estimate of the physics event in the target and helps cross-reference the event with the accelerator beam bunch and the incident photon's energy reading (discussed in Chapter 2). As a fast, rough counter of the charged tracks produced, the data from this device is important to the trigger.

Most of the GlueX detector systems are installed inside a $2.2 \mathrm{~T}$ solenoid magnet, constructed out of four super-conducting coils. The high field is important for analyzing charge particle momenta and for suppressing the low energy electromagnetic background generated by the high rate beam near the beam pipe. The polar angle coverage of the detector is segmented into the forward and barrel systems. The forward charged particles (below $\sim 10^{\circ}$ ) are tracked with the Forward Drift Chambers (FDC) and the forward-going gammas are detected in the Forward Calorimeter (FCAL). At larger angles, the detector is equipped with

\footnotetext{
${ }^{3}$ Indeed, the $1 / E$ distribution of bremsstrahlung photons results in a largely low-energy unpolarized flux on the target. Rejecting these is an essential requirement of the trigger system.
} 
the Central Drift Chamber (CDC) and the Barrel Calorimeter (BCAL). Tracks in the range of $10-20^{\circ}$ are reconstructed with hits in both the FDC and the Central Drift Chamber (CDC). The following sections will describe these systems and other detector components in more detail.

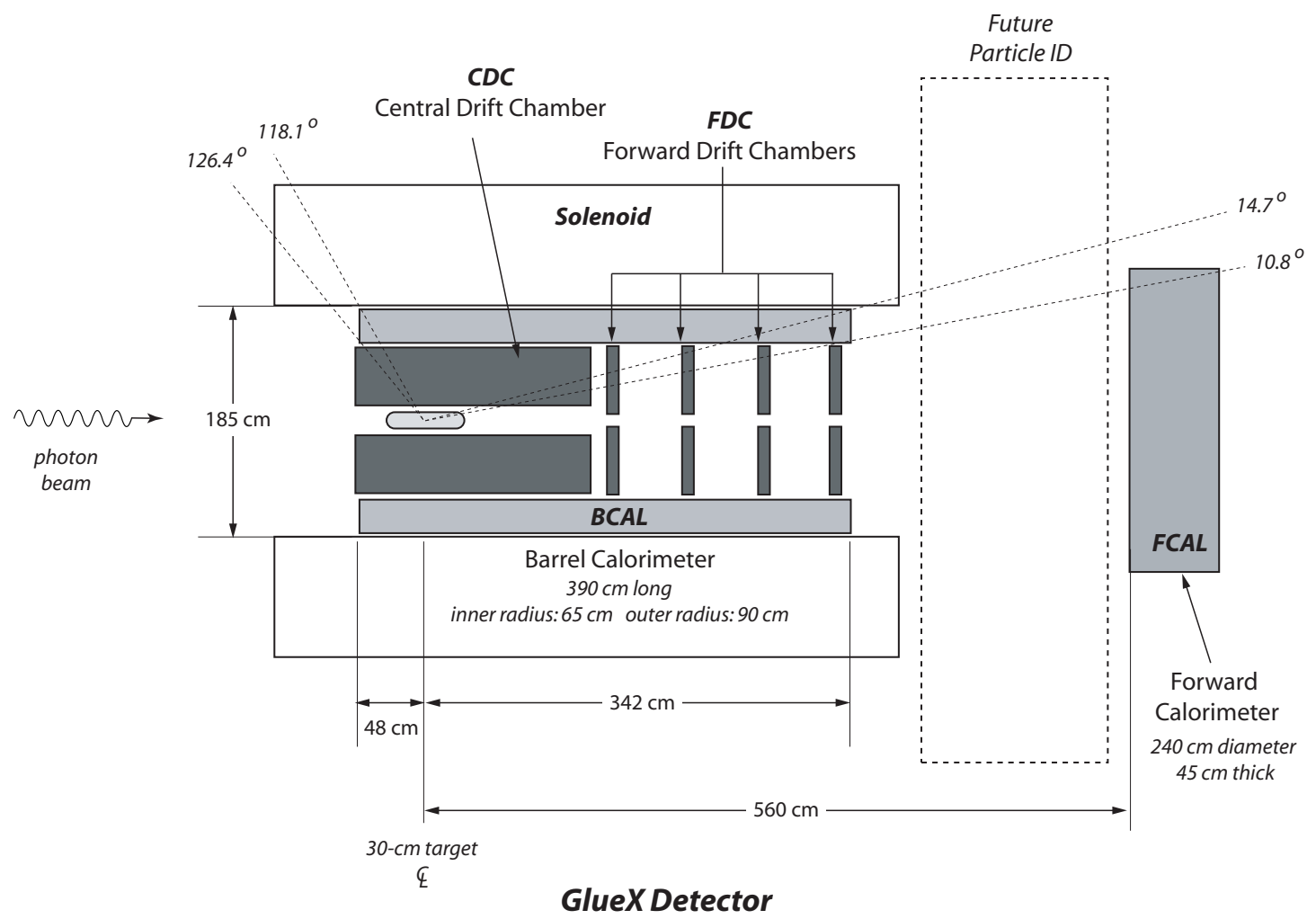

Fig. 1.2: Schematic cut-away view of the GlueX detector. Not visible is the time of flight system: a thin wall of scintillating counters stands in front of the FCAL with Start Counter instrumented with scintillators around the target. Barrel Calorimeter's fast readout contributes timing information at large angles. This forms the baseline design of the detector, with further PID detector systems under consideration.

\subsubsection{Tracking}

The CDC is a straw tube drift chamber: ionization left behind by a charged particle drifts toward a wire along the straw's axis that is held at high voltage. 

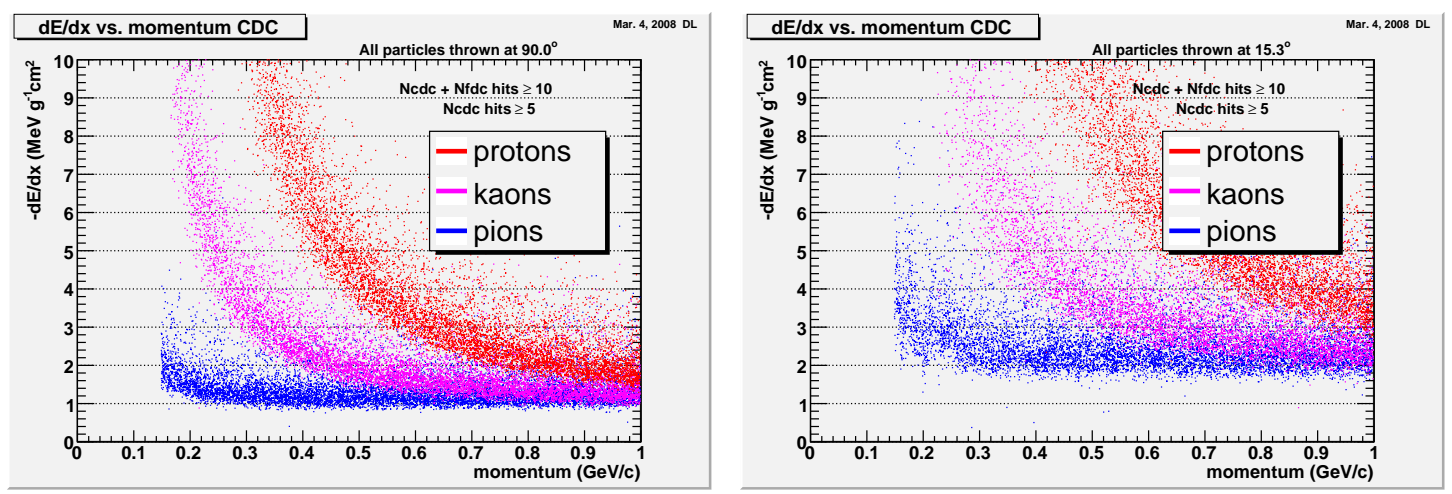

Fig. 1.3: These plots show the distributions of simulated $\mathrm{dE} / \mathrm{dx}$ measurements for pions, kaons and protons launched at $90^{\circ}$ (left) and $15^{\circ}$ (right) from the target. Good separation between these particles appears possible at moderate momenta, especially at higher angles, where more straws are traversed.

Measurement of the drift time allows for spatial resolution much better than the radius of the tube itself. The chamber is assembled in radial layers with 12 axial and 16 stereo layers oriented at $6^{\circ}$ with respect to the beam axis. Simultaneous fitting in all pulsed straws during event reconstruction resolves solutions with full three-dimensional particle trajectories. Simulation and prototype tests have demonstrated that the detector can meet the required $150 \mu \mathrm{m}$ radial and $1.5 \mathrm{~mm}$ axial hit resolution. The system should achieve momentum resolution of $1.5-3 \%$. Additionally, the CDC offers a measurement of the charged particles' $d E / d x$ - the rate of energy loss per unit distance traveled. For a given momentum, this rate depends on a particle's mass adding information about the particle's identity. The quality of separation of pions, kaons and protons is shown in Figure 1.3. The detector should allow for decent particle identification at large angles and low momenta, which is especially important for particles spiraling within the chamber without hitting the calorimeter.

Four FDC packages handle tracking in the forward direction. These are 
assembled from interleaved planes of field/sense wires and planes of cathode strips oriented transverse to the beam axis. Every such layer group is offset in the azimuth by $60^{\circ}$ to ensure unique identification of charged particle crossing points (i.e. eliminate "ghosting"). The goal is also to get many accurate points per track segment in the FDC package, including curvature information to help reconstruct the full track from these segments. These chambers should be able to accomplish $x, y$ position resolution of $200 \mu \mathrm{m}$.

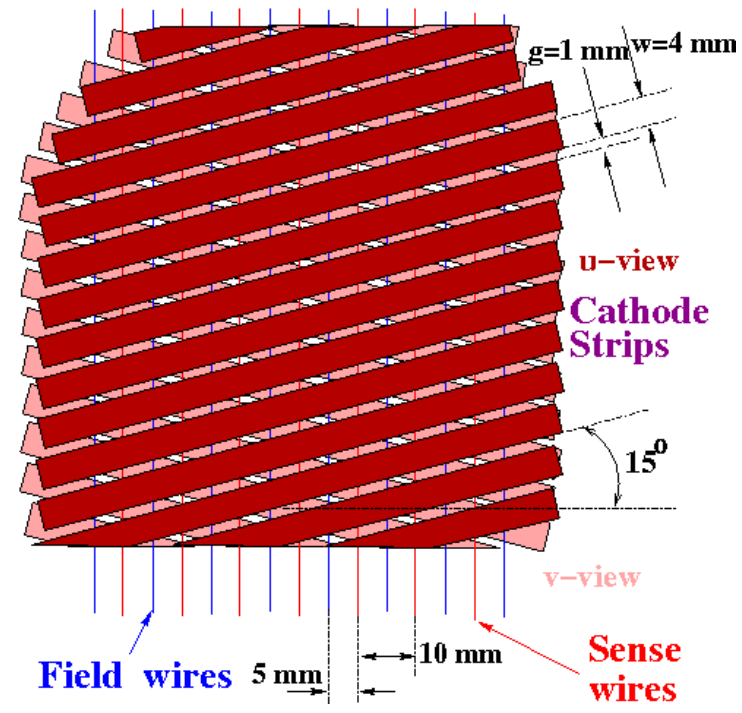

Fig. 1.4: This figure shows the scheme of forward drift chamber wiring. Note the alternation of field and sense wires between the cathode strips to create drift cells for ionization timing. The shown relative angular offset of the cathode strips and the $60^{\circ}$ shifts of similar assemblies in the construction of an FDC package help disambiguate the FDC crossing points.

\subsubsection{Calorimetry}

The barrel calorimeter (BCAL) measures the incident particle energy at angles above roughly $11^{\circ}$. The detector is composed of azimuthal sectors lining the bore of the solenoid magnet. Scintillating fibers run the length of the sectors 
(parallel to beam axis) to sample showers induced in the interstitial layers of lead. This "spaghetti" calorimeter is thus a matrix of scintillator, lead and glue, properly balanced and aligned. Beam tests have verified an energy resolution $\sigma_{E} / E=5.54 / \sqrt{E}$ convolved with a constant $1.6 \%$ contribution. Scintillators read out by the fast silicon photomultipliers (SiPMs) allow for timing and zcoordinate determination $(70 \mathrm{ps} / \sqrt{E}$ and $5 \mathrm{~cm}$ respectively). In spite of the short distance from the target, the timing measurement contributes to the time of flight determination with particle ID determination quality as shown in Figure 1.5. Gradual sampling of showers in the radial direction also adds data on $d E / d x$.
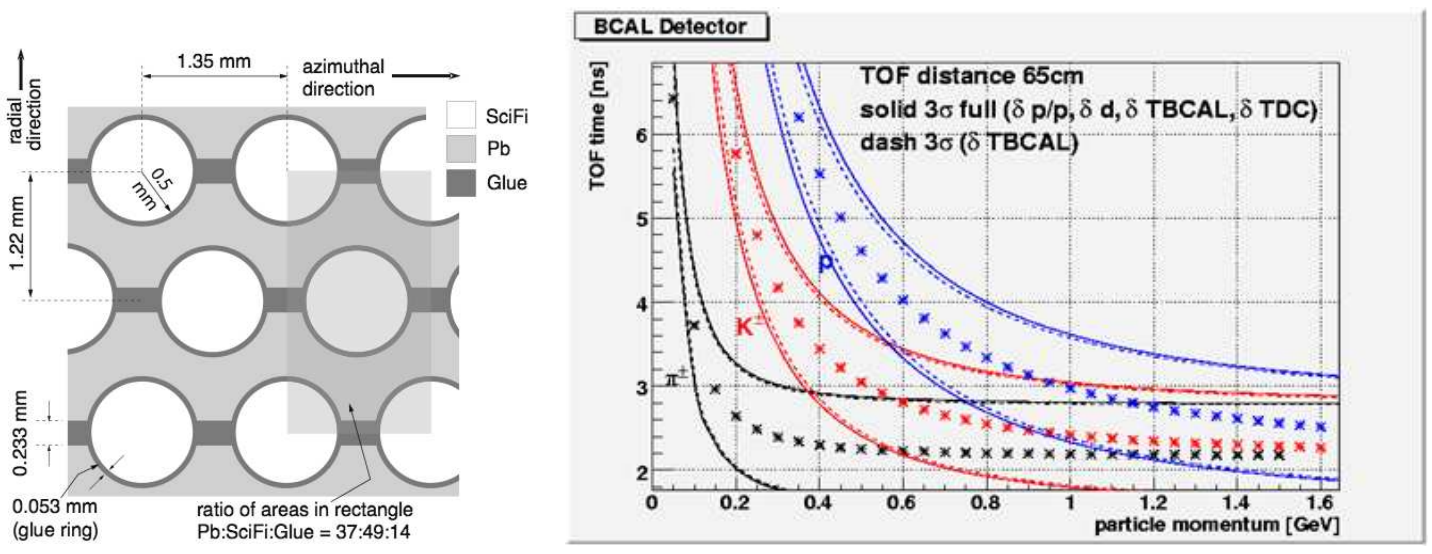

Fig. 1.5: The scintillator and corrogated lead sheet layering pattern for the barrel calorimeter is shown on the left. The expected time of flight measurement uncertainties as a function of momentum for different charged particle species is shown on the right.

The forward calorimeter (FCAL) is composed of F8-00 lead glass blocks of dimensions $4 \times 4 \times 45 \mathrm{~cm}$ oriented along the beamline. This shower medium has been used in previous experiments and with new photo-tubes and bases, it should achieve energy resolution of $\sigma_{E} / E=5.7 / \sqrt{E}$ with a constant $1.6 \%$ contribution. Timing algorithms running on the digitizing electronics will help resolve shower timing to within $150 \mathrm{ps}$, reducing the background due to pileup in this calorimeter. 


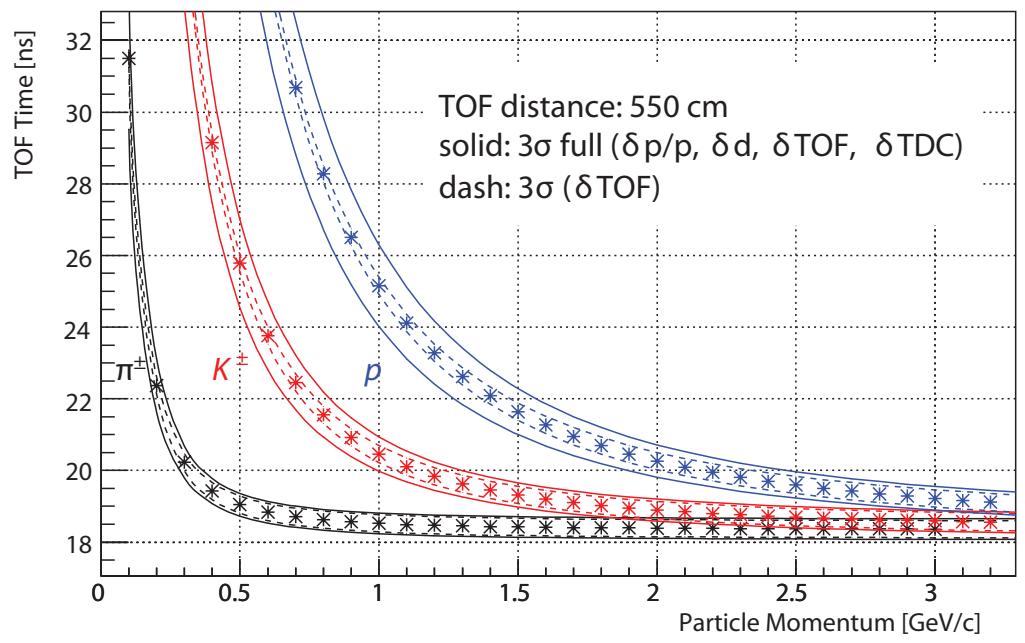

Fig. 1.6: This plot shows the expected TOF wall resolution of pions, kaons and protons, assuming 3\% momentum uncertainty, a path length uncertainty of $1 \mathrm{~cm}$, and time resolution from the detector and readout of $80 \mathrm{ps}$ and $40 \mathrm{ps}$, respectively.

\subsubsection{Particle Identification}

When determination of a particle 4-vector is not precise enough to distinguish the particle specie by mass, extra information is needed from a dedicated particle identification (PID) system. Conversely, a more precise determination of the 4-vector is possible with the constraint of the particle mass. At moderatelyhigh energies, an independent measurement of particle velocity in addition to momentum can reveal its mass. The baseline design of the GlueX detector includes a time-of-flight (TOF) system, comprised of timing in the BCAL as well as a dedicated wall of scintillation counters in the forward direction just in front of the FCAL. A sub-100 ps resolution TOF wall is made of cross-hatched paddles read out on both sides for good timing resolution and position sensitivity. The expected performance, based on simulation and cosmic tests with prototype paddles, is shown in Figure 1.6 [37]. 


\section{Chapter 2}

\section{Photon Beamline}

\subsection{Photon Beam}

An effective method for creating a high intensity photon beam is through bremsstrahlung of an electron beam in a dense radiator. A broad spectrum of photons is produced with this method, but the energy of the photon participating in an event in the detector can be measured. This is done by spectrally analyzing the beam of post-bremsstrahlung electrons that emerge from the radiator using a dipole magnet and a set of fast counters. The counters record the timing of an electron that emerges from the magnetic field to reference it to the beam bunch of its origin. The energy is automatically determined from the location of the counter, since the beam origin, the known magnetic field and the counter end point fully define the trajectory of the electron. Knowing the initial electron beam energy, the produced photon is thus "tagged" with a known energy. ${ }^{4}$ A schematic of this setup as designed for Hall D is shown in Figure 2.1. The quadrupole magnet shown in the figure is part of the optics that focuses the analyzed electron beam on the plane of the counters - the focal plane. The bremsstrahlung process tends

\footnotetext{
${ }^{4}$ In practice, the look-up table from counter location to photon energy will be calibrated with a pair spectrometer. This device employs a thin converter to induce pair production in a small fraction of high energy photons to measure their energy. The tagging counters are calibrated by running in coincidence with the pair spectrometer.
} 


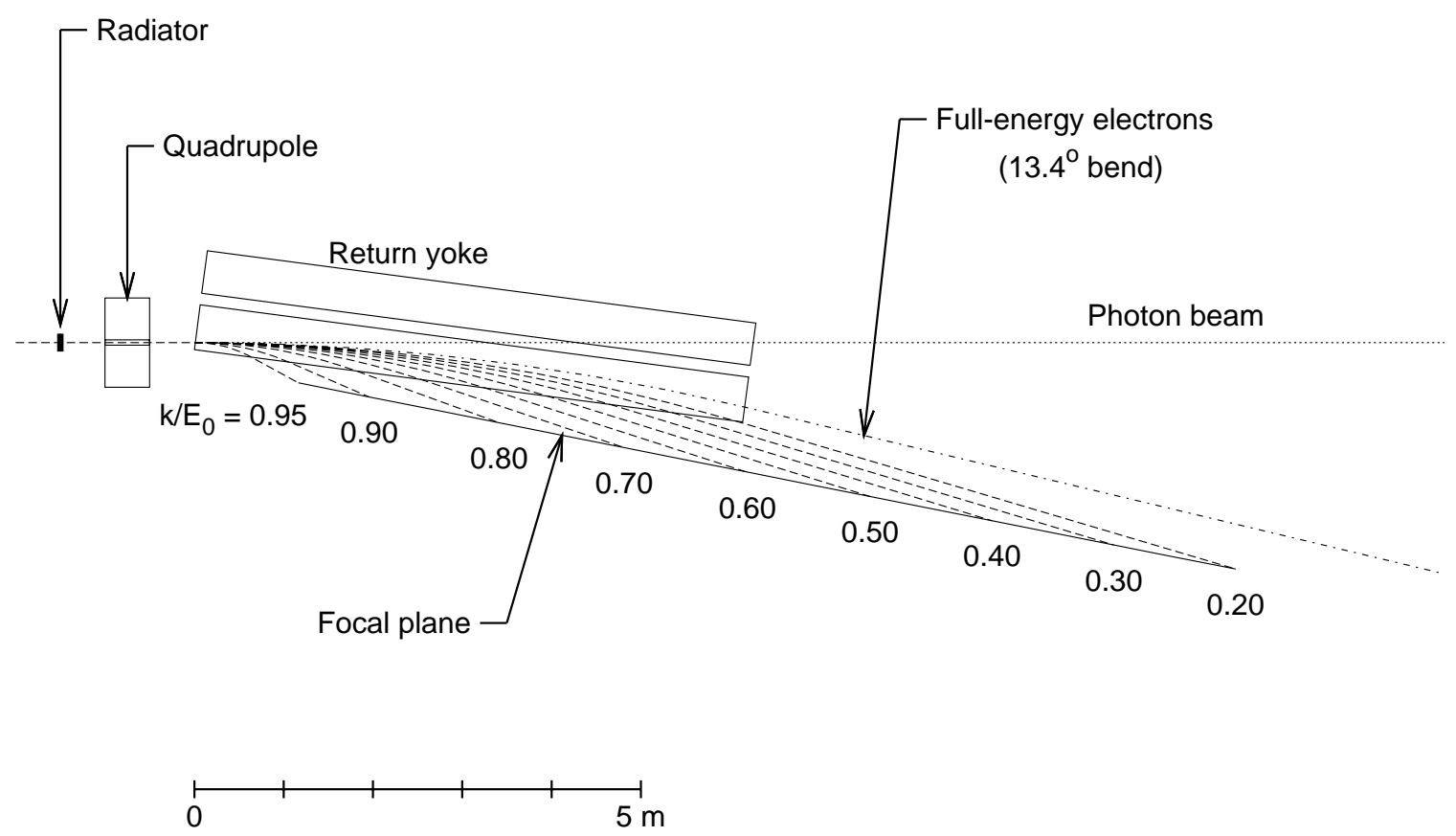

Fig. 2.1: Tagger hall instrumentation is illustrated with a horizontal section along the beam axis and the symmetry plane of the tagger dipole magnet.

to favor very soft photon emission, so the majority of the electrons emerge close to their full energy and proceed to the electron beam dump. A detector providing a high resolution set of counters, nick-named as the "tagger microscope" (TM) is one of the main subjects of this work and will be discussed in full detail in Chapter 3.

A polarized photon beam can be produced through bremsstrahlung of the electron beam in a mono-crystalline diamond radiator. The essence of this technique, called "coherent bremsstrahlung" (CB) is that an ordered crystal lattice oriented appropriately with respect to the electron beam recoils coherently from the incident electron. This produces an enhancement in the radiated photon spectrum corresponding to the aligned inverse lattice vector. Furthermore, this alignment defines a preferred plane of scattering, given by the inverse lattice and 
electron momentum vectors, emitting photons polarized in this plane.

The location of this enhancement in the bremsstrahlung energy spectrum is adjusted by orienting the radiator and can, in principle, be brought arbitrarily close to the end point. However, in the limit of total energy transfer from the electron, the momentum transfer vector is collinear with the incident electron momentum, so that the two-body scattering plane is no longer well-defined. This means that the photon beam is unpolarized at the end point. Thus, there is a trade-off between photon beam energy and polarization. Optimization early in the experiment's design between higher energy reach and polarization led to a decision of placing the primary $\mathrm{CB}$ peak at $9 \mathrm{GeV}$, corresponding to $40 \%$ polarization. Figure 2.2 shows the polarization of the $\mathrm{CB}$ spectrum from a $12 \mathrm{GeV}$ electron beam with the peak at $9 \mathrm{GeV}$ and the variation of the polarization fraction as a function of peak energy. The plot also shows the effect of collimating the beam a technique discussed in the next section.

Coherent scattering edges in the energy spectrum are dominated by a power law background (shown in Figure 2.4). Indeed, in addition to CB, the electron can also scatter incoherently as it would from an amorphous radiator. This occurs when the lattice does not recoil collectively (lowest order vibration mode) or due to lattice momentum components that deviate from periodic structure, like the form-factor of the atom and the smearing of their order from temperature and zero-point motion-induced vibrations. These effects drive the selection of the material for the radiator medium.

Roughly speaking, an ideal material contains strong transverse electric fields and has rigidly held lattice sites. These conditions ensure a good recoil medium that is very well ordered to ensure coherent scattering. The largest fields are deep 


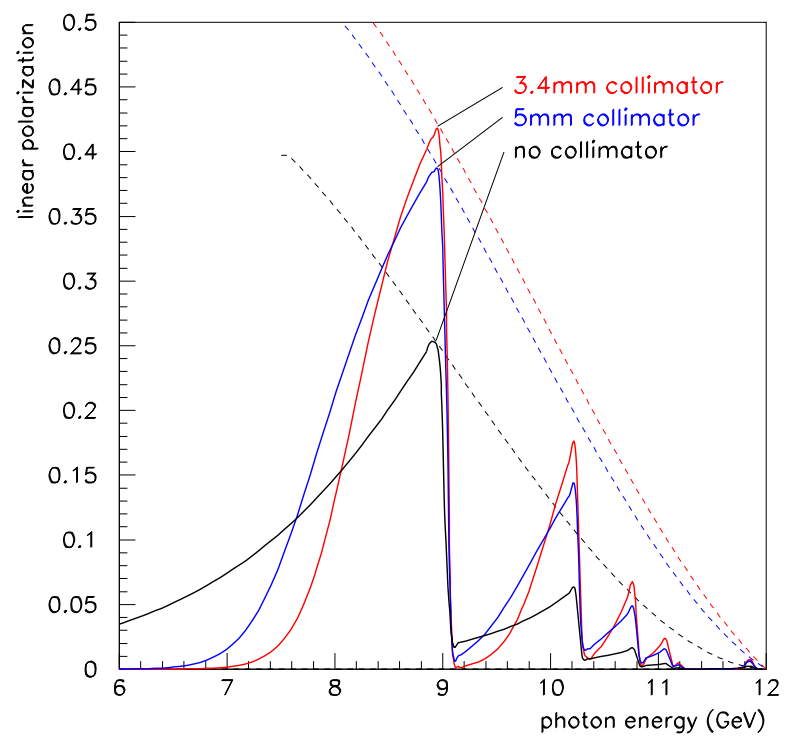

Fig. 2.2: Degree of linear polarization of the coherent bremsstrahlung photon beam as a function of energy, under varying collimation conditions. The dashed lines show the trajectory of the maximum polarization fraction as a function of the peak energy. These are shown for three collimator aperture scenarios.

inside the atoms, but on the scale of the crystal lattice, the fields are screened and the residual fields are very weak. An optimal crystal must be very tightly packed, to allow more of an overlap between the atomic and lattice form factors. This implies light elements; concerns for radiation hardness also urge against compound materials. A survey of materials ordered by their figure of merit defined as the product of atomic and crystal form factors arrives at just a few choices [5]. When considering the available expertise in fabrication, diamond is a clear winner.

The challenge in developing a diamond radiator that meets the Hall D requirements is ensuring electron momentum to inverse lattice vector matching across an area of several $\mathrm{mm}^{2}$. The electron beam emittance is taken as an irreducible source of electron momentum spread. The thickness of the radiator is 
minimized to reduce the contribution due to multiple scattering within constraints of maintaining a robust radiator against damage in the beam. The resulting specification is $\sim 10^{-4}$ radiation lengths or about $20 \mu \mathrm{m}$ thickness. The total electron momentum spread from these effects sets the scale for the spread of the inverse lattice vectors ("mosaic spread"). The final requirement on the whole-crystal mosaic spread is $20 \mu \mathrm{r}$. The achievable beam spot on the radiator (constrained by the optics requirements discussed in the next section) is $1.7 \mathrm{~mm}$ by $0.5 \mathrm{~mm}$ leading to a diamond wafer side length of $5 \mathrm{~mm}$. Further discussion of the radiator performance parameters and the work conducted by the author on diamond characterization is presented in Section 2.1.3.

\subsubsection{Collimation}

Coherent bremsstrahlung implies that the electron effectively undergoes two-body scattering from the crystal lattice. Energy and momentum conservation in this process lead to a correlation between emission angle and the energy of the produced photon. However, the electron can also scatter incoherently as it would from an amorphous radiator. This occurs when the crystal recoils in higher order modes instead of collectively. Scattering that proceeds incoherently will not have a correlation between angle and energy. The distribution in polar angle and energy for both of these processes is given in Figure 2.3. The shape of the coherent bremsstrahlung distribution allows us to reduce the incoherent background significantly under the coherent edge, by imposing an angle cut. Therefore, the Hall D facility will use collimators to improve the fraction of coherent photons to boost the polarization fraction.

However, a collimator presents strictly an angular cut in photon emission 


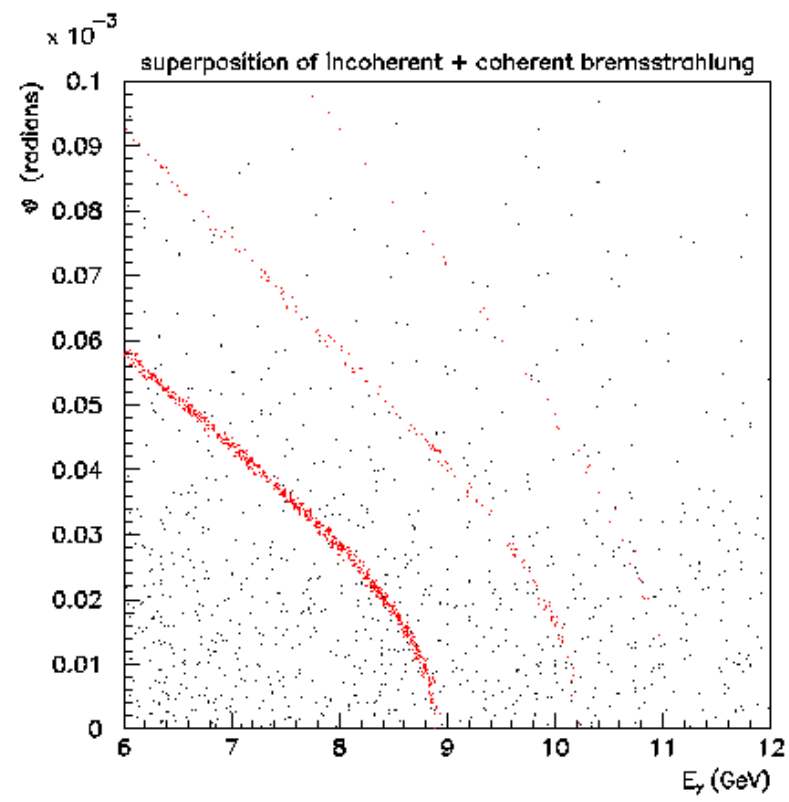

Fig. 2.3: Distribution of bremsstrahlung photons in polar angle and energy, with the coherent part shown in red bands.

angle only if the original electron beam had zero emittance. Otherwise, limiting transverse acceptance also results in a cut in beam size or electron beam divergence angle (depending on the electron beam optics.) To optimize a collimator's ability to limit the photon emission angle, the electron beam must be tuned as follows:

1. minimal achievable emittance

2. beam focus on the collimator face

3. gradual convergence angle toward the focus

4. large enough distance to the collimator for photon beam divergence to dominate the electron's

The gradual convergence angle ensures that the coherent edges are not significantly smeared due to a spread in electron beam angle with respect to the 


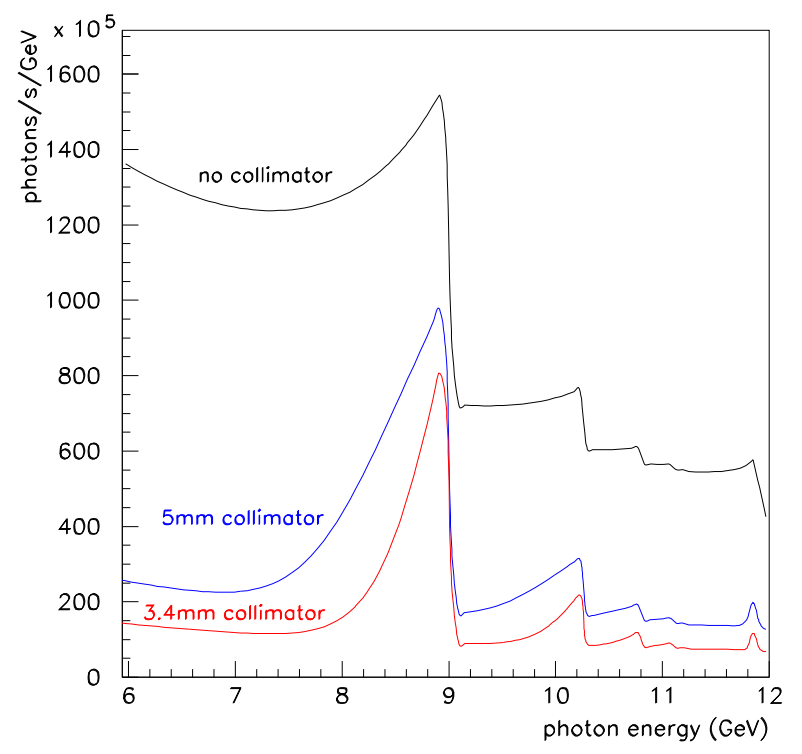

Fig. 2.4: Simulated photon spectra with various degrees of collimation.

crystal lattice planes. Focusing at the collimator at large distance ensures that the collimator is least sensitive to the electron beam divergence and maximally sensitive to the photon's.

More quantitatively: let us consider the "mosaic spread" (to be discussed later) of about $20 \mu \mathrm{r}$ and set the electron beam convergence angle (r.m.s. beam envelope with respect to beam axis) to the same. Taking an achievable emittance of $10 \mathrm{~mm} \cdot \mu \mathrm{r}$ leads to a $0.5 \mathrm{~mm}$ spot on the collimator. Now, making sure that the collimator size is much greater than the beam spot, at least a factor of three: a $1.5 \mathrm{~mm}$ radius aperture with collimation of $\frac{1}{2} \frac{\mathrm{m}}{E}$ results in a minimum collimator distance of $70 \mathrm{~m}$. This result was among the driving decisions to build a new facility, with an experimental station far enough away to accommodate the proper optics. Figure 2.4 shows the effect of introducing collimation of various degrees. The improvement in the purity of the CB beam is substantial. 
There are several challenges consequent to this collimated photon beamline design. One of these is the difficulty of stabilizing the beam on a millimeterscale aperture $\sim 70 \mathrm{~m}$ away from the electron beam optics. Implementation of a photon beam position sensor with feedback to the steering magnets is discussed in Section 2.1.2. The second major challenge is the reduction of tagging efficiency: electrons corresponding to stopped photons still end up incident on the tagger counters. This contributes to higher rates (lower counting efficiency) and higher tag ambiguity due the already high accidentals rate. The solution to this problem is to restrict electron detection on the tagger in a way that mimics the photon collimator acceptance. This feature of the tagger hodoscope system is discussed in Chapter 3.

\subsubsection{Acitve Collimator}

Stabilization of charged particle beam orbits relies on beam position monitors for feedback. Projecting a photon beam a significant distance after the electron beam stabilizers requires similar feedback. In particular, the Hall D beamline requires collimators with apertures of a few millimeters in diameter, which are effective only if the beam is threaded precisely through their axis. Thus, the project adapted a specification of beam position stability within $200 \mu \mathrm{m}$. In a sense, the first collimator must be made sensitive to the incidence position of the beam, or become an Active Collimator.

The technology for this has been developed at SLAC: four tungsten plates are arranged around the beamline to detect the drift of the beam from the center hole. The signal of beam incident on a plate comes from the emitted knock-on and Compton electrons from the showers that develop in the dense material [31]. 
Comparison of the currents from the opposing plates provides a measure of the beam asymmetry. The SLAC team has optimized the geometry of the plates, prescribing tungsten plates of 2 radiation lengths in thickness, with 4 radiation length tungsten pins raised from the downstream-facing side of the plates. The solid plates facilitate the development of the shower, while the pin stage offers more open geometry for electron emission. Implementation of this technique for the Hall D beamline involves two concentric rings of these tungsten "pin cushions". The outer ring provides coarse position information, covering the radial range of $3-6 \mathrm{~cm}$. The inner ring provides the actual, fine, real-time centering information, covering $2.5 \mathrm{~mm}-2.5 \mathrm{~cm}$ range. Note that the sensitivity of this device does not begin at the radius of $2.5 \mathrm{~mm}$ : the tails of the beam would provide sufficient overlap with the plates to ensure continuous sensitivity to the beam position.

Simulation of this design showed currents on the order of $10 \mathrm{pA}$ when the beam is centered, rising by two orders of magnitude when it shifts significantly into the area of a tungsten plate. This gives a sense of the current readout requirements. So, to instrument this detector, the current collector electrode (ground) was designed as a cup with separate compartments enclosing each of the tungsten pin-cushions. The pin-cushions themselves are mounted on a boronnitride surface that is covered by another grounded plate to create a full Faraday cup around the tungsten pieces [19]. The full assembly is shown in Figure 2.5. A very high gain current amplifier was clearly needed for this detector.

The bandwidth of this beam position monitor is very important to the overall beam stabilization system. The detector must be fast enough to detect all beam motion Fourier components whose sum takes the r.m.s. position above the specified $200 \mu \mathrm{m}$. The $60 \mathrm{~Hz}$ component and a few of its multiples represent one 


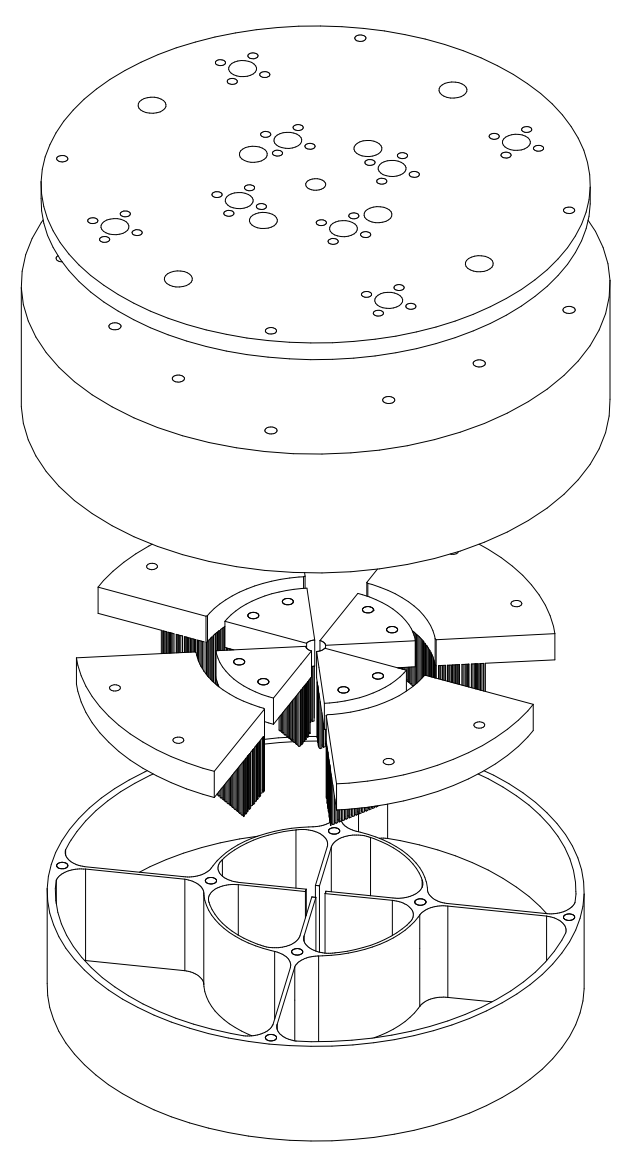

Fig. 2.5: Exploded view of the Active Collimator assembly. In this view the beam entry is from the top. From top to bottom, the layers are: face plate, boron-nitride insulating cup, tungsten "pin-cushions", grounded cup. The top and bottom pieces form a grounded Faraday cup to shield the detector.

obvious source of beam motion. A rough goal of approaching $1 \mathrm{kHz}$ bandwidth has been set for this design. Typically, an amplifier is characterized by its gainbandwidth product, so bandwidth would come at the expense of amplification.

The design and the majority of fabrication of this detector was finished at the start of the author's work on this project. The remaining tasks included:

1. completion of the faceplate design with a scheme for connecting to the tungsten pieces 


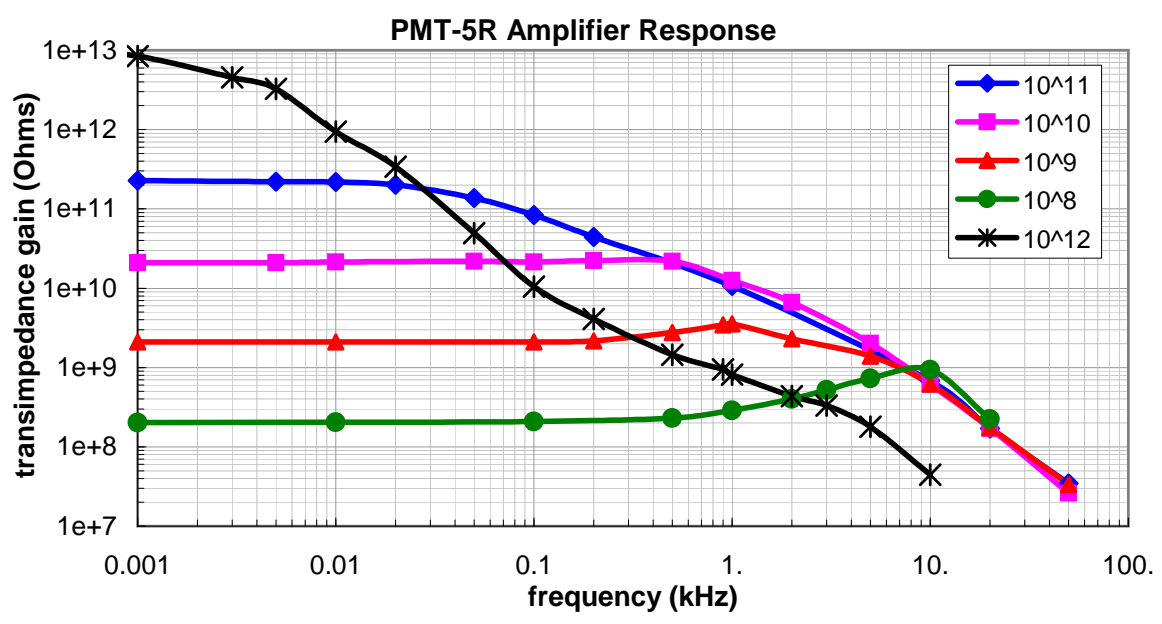

Fig. 2.6: Plot of the five highest gain settings of the Advanced Research Instruments PMT-5R amplifier, labeled by their nominal gain settings. The vertical scale shows higher values than advertised and may suffer from a systematic normalization error. The relative bandwidths of the gain modes is the most important result.

2. characterizing the high-gain current amplifiers

3. instrumentation for and conduction of a beam test

The Advanced Research Instruments PMT-5R amplifier was selected for this instrument. The amplifier offers digitally-selectable gain modes ranging from $10^{6}-10^{12} \Omega$. Its response was independently tested on the bench with attention to its frequency spectrum. The results are shown in Figure 2.6. Even higher than advertised gain settings were found at DC, however there may be a systematic normalization error contributing to this. But the principle result is the relative frequency response between the modes, which is telling of the trade-off between gain and bandwidth. Clearly, the lowest usable gain should be used with this instrument and adjusted online depending on the run conditions.

The author's most significant engagement with this project was the design of control and readout software for beam-testing of the Active Collimator. The 
beam tests were performed just upstream of the photon beam dump in Hall B of Jefferson Lab. The device was attached to the translation stage of the total absorption counter used for beam flux normalization in that facility. The test involved moving the Active Collimator with respect to the already stabilized photon beam to simulate the relative misalignment of the beam and the instrument. The software, written as a LabView virtual instrument, integrated the facility's information on beam conditions, moving and recording data from the Active Collimator accordingly.

Signals from the instrument's channels were acquired for several seconds at each position in order to perform a high-resolution Fourier analysis of the results. Equally long exposures were taken on the bench and in the Hall without beam, in order to understand the contribution from noise. Several runs were conducted with $15 \mathrm{nA}$ electron beam current on a $10^{-4}$ radiation lengths converter. This is the same radiator thickness as what is intended for Hall D and an order of magnitude lower current than the low intensity $100 \mathrm{nA}$ current planned for Hall D. Success in these conservative conditions will assure robust operation in the new facility.

Figure 2.7 shows the steady-state current from the opposing tungsten plates in the Active Collimator as a function of displacement with respect to the photon beam. The cross-talk, what looks like inverted copies of signals from neighboring channels, has been understood as a consequence of an improper connection - the outputs were connected together at the end of a long cable path of some resistance. This will not be done in the final system.

The crossing of the signals from the inner tungsten plates shows sensitivity as a function of position. A better illustration, given in Figure 2.8 shows the current asymmetry between the two inner tungsten plates as a function of position. 

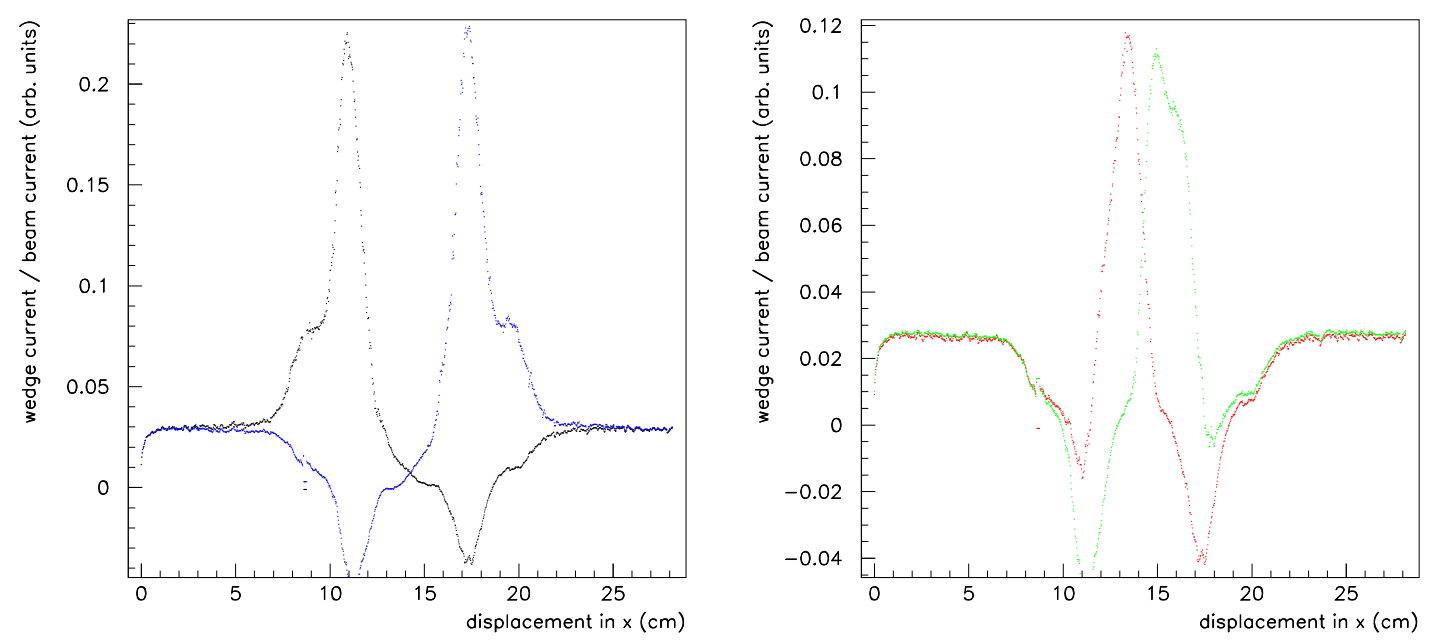

Fig. 2.7: DC current from the outer (left) and inner (right) tungesten wedges as a function of the Active Collimator's displacement with respect to the beam. The apparent cross-talk has been understood as a symptom of an improper connection, which should not appear in the final system.

Figure 2.9 shows the Fourier transform averaged from $1001 \mathrm{~s}$ traces taken from an inner wedge at incremental positions between $-2.5 \mathrm{~mm}$ to $+2.5 \mathrm{~mm}$ from the beam center. (The clearance hole for the beam is $5 \mathrm{~mm}$ in diameter.) Multiples of the $60 \mathrm{~Hz}$ component are clearly visible. Note, that because data was always taken with the Active Collimator at rest, these Fourier components indicate the remaining photon beam motion in Hall B. This data was taken in the $10^{10}$ gain mode and confirms the bandwidth measured on the bench (Figure 2.6) very nicely. The bandwidth demonstrated here is already sufficient for the requirements of Hall D. Additionally, the higher beam current in Hall D may allow use of the $10^{9}$ gain mode, which would win another order of magnitude in bandwidth. The detector noise floor that is well below the $200 \mu \mathrm{m}$ indicates that this stability specification will be met with this detector. 


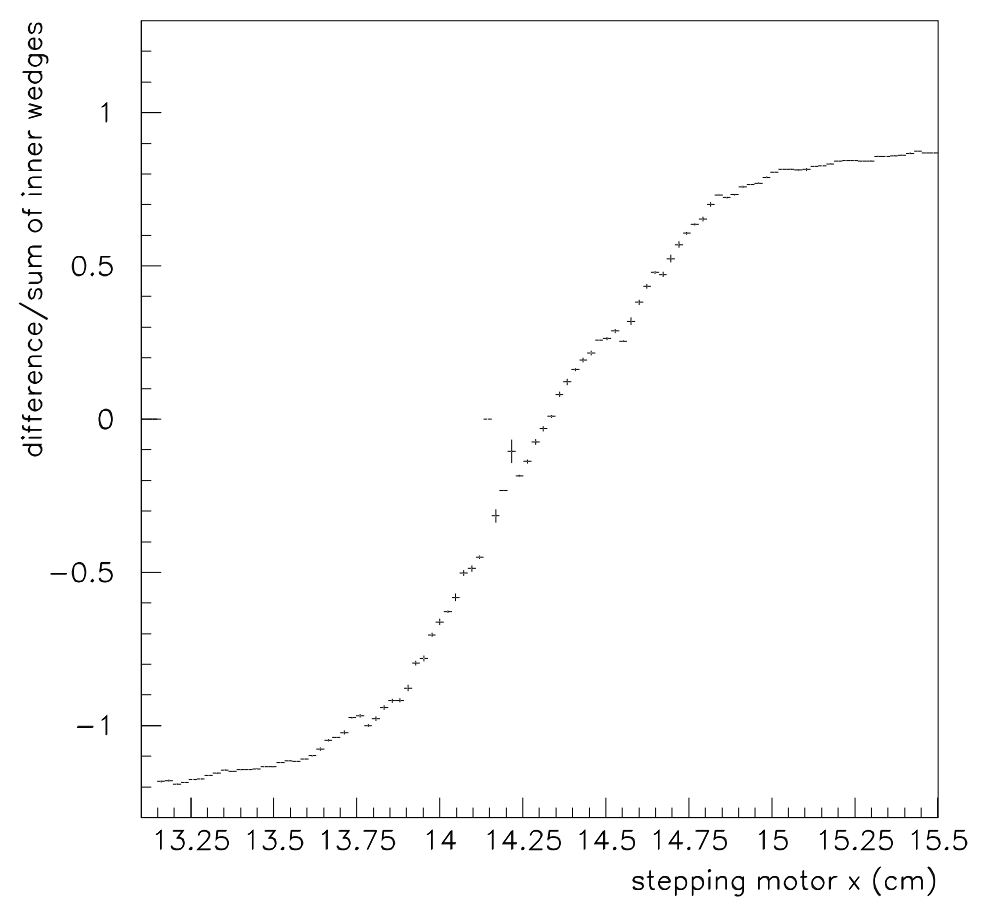

Fig. 2.8: The DC current asymmetry defined as the difference of the sum of the two inner tungsten plates as a function of beam position.

\subsubsection{Diamond Characterization}

As discussed earlier, significant variations in the scattering plane orientation across the diamond surface ("mosaic spread") or in the electron trajectory due to multiple scattering result in degradation of $\mathrm{CB}$ fraction in the photon output and consequently the polarization fraction. Thus, what is required is a diamond radiator that is thin enough to minimize multiple scattering while remaining strong enough to last in a high intensity beam and maintain small mosaic spread. Prior experience with thin diamond bremsstrahlung radiators and simulations of the Hall D beamline lead to a specification of $20 \mu$ r mosaic spread and maximum possible thickness of $20 \mu \mathrm{m}$.

Synthetically-produced diamonds using the processes of High-Pressure High- 


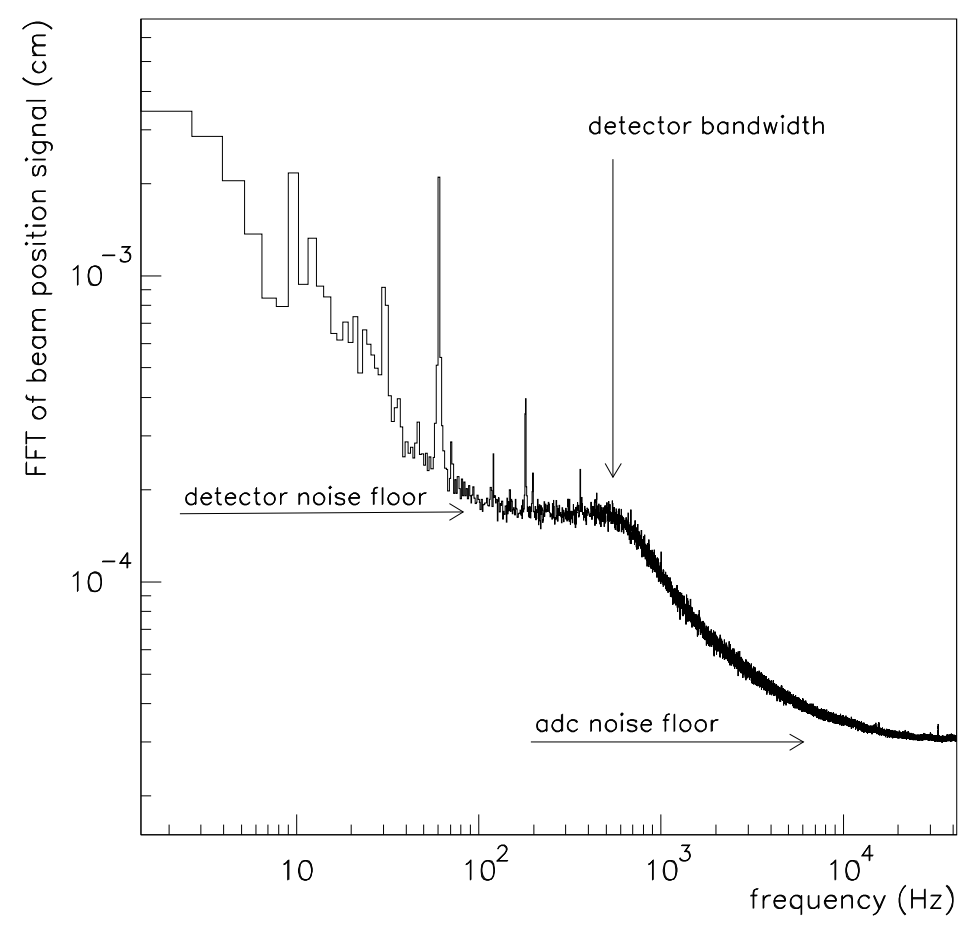

Fig. 2.9: The Fourier transform averaged over $1001 \mathrm{~s}$ traces from inner tungsten pieces taken at incremental positions from $-2.5 \mathrm{~mm}$ to $+2.5 \mathrm{~mm}$ from the beam center. The bandwidth corresponds well to that measured on the bench for the $10^{10}$ gain mode.

Temperature (HPHT) and Chemical Vapor Deposition (CVD) are known to result in crystals with mosaic spread that meets the requirement. Unfortunately, the samples that usually emerge from these processes are on the order of $300 \mu \mathrm{m}$ thick. Thinning these wafers without degrading their mosaic spread is a significant challenge. Most mechanical techniques to date introduced significant internal strain and consequent warping of the wafer. A diamond thinned without these strains to the scale of $20 \mu \mathrm{m}$ may still be susceptible to flexing from the way it is mounted and from vibrations. Some new techniques have emerged for thinning diamond, such as reactive chemical mechanical polishing (RCMP), reactive ion etching and ultraviolet laser-ablation. The first two techniques allow a gentler erosion process 
that may avoid introduction of strain, but they still produce a uniformly thin diamond that is vulnerable to flexing once mounted in the beamline. Laser ablation allows selective thinning of the diamond surface: a thin area large enough for the electron beam spot can be created in the center while leaving the wafer's edges thick enough for mechanical reinforcement. Reactive ion etching is notable for offering a very rapid thinning technique and it may yet offer selective thinning similar to laser ablation by using metallic masks to protect the wafer edges. This, however, has not been demonstrated as of the time of this writing.

Paramount in the search for good, thin diamond radiators is a technique for their characterization. Transmission-mode X-ray scattering is thought to be the most thorough for assessing the mosaic spread of a crystal. A sample is oriented with respect to a monochromatic hard X-ray beam to meet the Bragg condition for a desired crystal plane. ${ }^{5}$ The distribution in angle over which this condition is met is determined by rocking the sample in the angle of scattering and measuring the scattered X-ray output. This "rocking curve" measurement can be performed on the entire wafer at once by illuminating the entire sample and measuring the scattered radiation with a high-resolution X-ray camera. The median angle at which the Bragg condition is met is determined from the centroid of the local rocking curve, while the local mosaic spread is determined from its width. The centroid and width of the rocking curve are often functions of position on the diamond wafer as a result of variations in crystal quality, local strains and macroscopic wafer curvature. Thus, this is a great technique for analyzing all the relevant performance parameters of a radiator sample.

\footnotetext{
${ }^{5}$ Coherence of scattered waves from a desired family of crystal planes is ensured by Bragg's Law: $2 d \sin \theta=n \lambda$, where $d$ is the lattice plane spacing, lamba is the X-ray wavelength and $n$ is an integer allowing phase lag in multiples of $2 \pi$.
} 
The author's involvement with this project included characterization of various diamonds before and after thinning using some of the early available thinning techniques. ${ }^{6}$ The X-ray crystallography took place at the Cornell High Energy Synchrotron Source (CHESS) C-line. This station receives synchrotron X-rays from a bend magnet $14.5 \mathrm{~m}$ away that steers a $5.5 \mathrm{GeV}$ electron beam. The angular divergence is $m / E$ or about $100 \mu \mathrm{r}$. This is too large to resolve the soughtafter diamond rocking curve of $15 \mu \mathrm{r}$. The beam is also "white" - ranging roughly $1-50 \mathrm{keV}$, but what is needed is a monochromatic beam to select a nominal angle that meets the Bragg condition and examine the angular spread with respect to this point. This problem is nicely solved with a set of asymmetric double-bounce monochromators. The beam is reflected from two silicon crystal surfaces rotated to match the (331) lattice planes and select $15 \mathrm{keV}$ X-rays. The asymmetric cut of the monochromator crystals was made to expand the beam from its initial $1.5 \mathrm{~mm}$ r.m.s. in the vertical direction by 8 times. This will ensure full coverage of diamond samples for imaging the rocking curves over the full surface at once. Analogously to beam expansion in visible optics, this beam expansion decreases the divergence by the same factor. At the end of the monochromator setup, the beam is characterized by performing a rocking curve measurement on a third silicon crystal aligned to match the same (331) lattice plane. This measurement yielded a width of less than $10 \mu \mathrm{r}$, which is understood to be a combination of the remaining beam divergence, the Darwin width of this crystal plane (about $5 \mu \mathrm{r}$ ), finite spacial resolution and test silicon wafer curvature. It was therefore clear that this beam instrumentation was sufficient for diamond characterization.

\footnotetext{
6 The author was involved in two measurements: May of 2009 and 2011. Most of the following discussion pertains to the second run, but incorporates many of the lessons learned from earlier measurements.
} 
Figure 2.10 shows the rocking curve r.m.s. width and centroid as a function of position on a $300 \mu$ m-thick electronic grade diamond wafer from Element-6. Previous scans have shown such products to be very rigid and mono-crystaline, resulting in full crystal rocking curves acceptable to GlueX. This sample was scanned to compare its present characteristics to those it will have after it is thinned. Figure 2.11 shows the rocking curve width and centroid as a function of position on a CVD type 3A SC "plate" surface. This significantly cheaper material that is considered "non-electronic grade" shows surprisingly good crystal quality. This may be a candidate material for future thinned diamonds. Figure 2.12 presents the same plots for a thinned electron grade mono-crystal. Comparing these sets, it is evident that apart from "hot spots" where significant structural damage must have occurred during thinning, the local crystal structure maintains a small mosaic spread. However, the centroid of the rocking curves, corresponding to the local normal to the scattering planes, is broadly distributed in the thinned sample. The virgin wafers appear to maintain the crystal orientation rigidly, whereas the thinned diamond loses its planar stiffness and perhaps develops internal strains that result in a curved natural shape. Given that the local mosaic spread after thinning remains small, it seems that wafer flatness is now the primary challenge. Similar conclusions may be drawn from the $10 \mu \mathrm{m}$ Element 6-thinned diamond (Figure 2.13). While the local crystal structure is sufficiently good across most of the surface, the crystal plane orientation differs widely across the diamond surface. Thus, the average over a spot of several $\mathrm{mm}^{2}$ would result in an unacceptably-wide mosaic spread. 

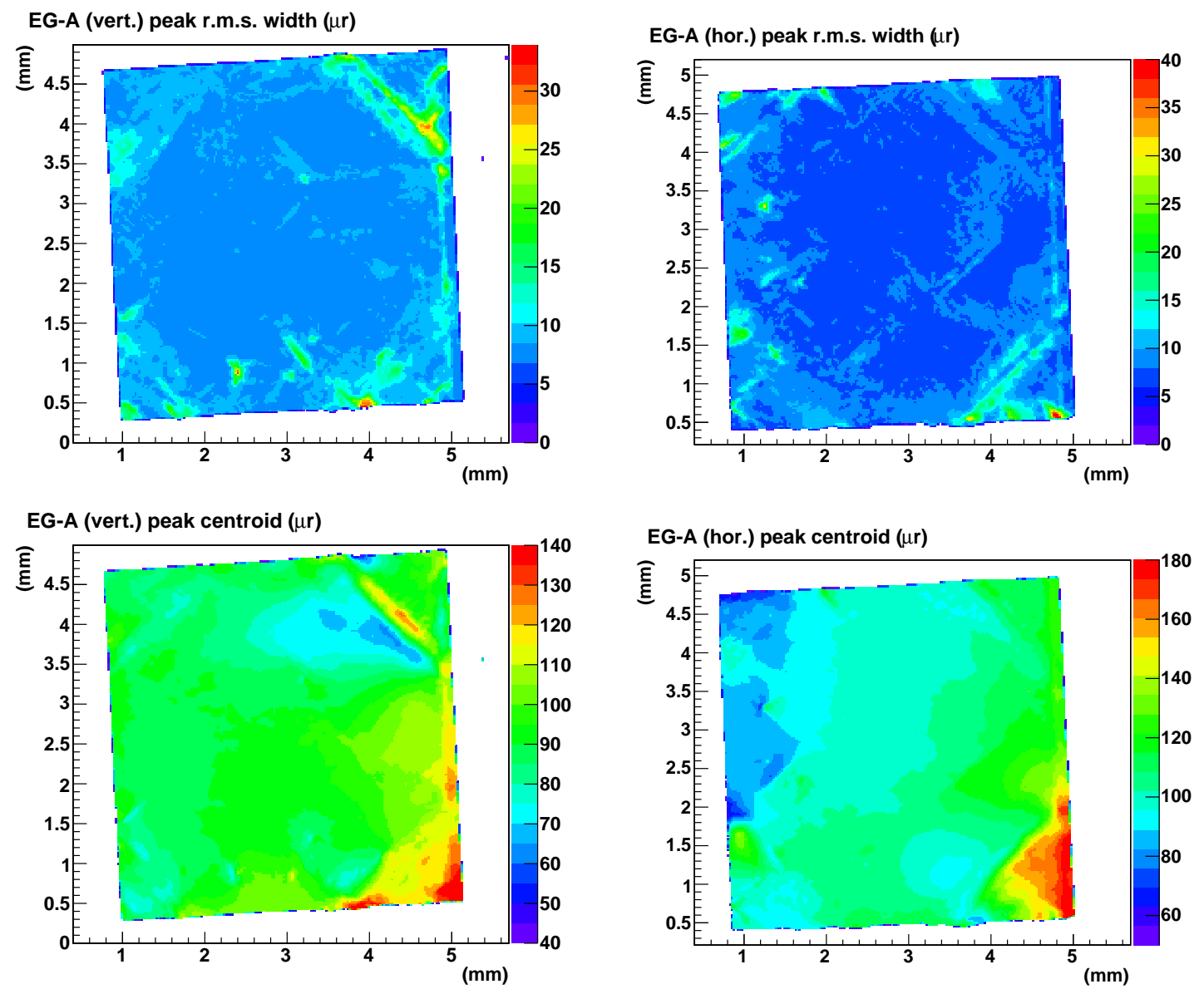

Fig. 2.10: Top row: Plots of local rocking curve width for an electron grade wafer in the vertical and horizontal scan orientations. Bottom row: Corresponding plots of the centroid of the local rocking curves. This $300 \mu \mathrm{m}$-thick, virgin sample clearly holds its normal to the scattering plane uniformly across the surface. 
Plate B (vert.) peak r.m.s. width ( $\mu$ r)

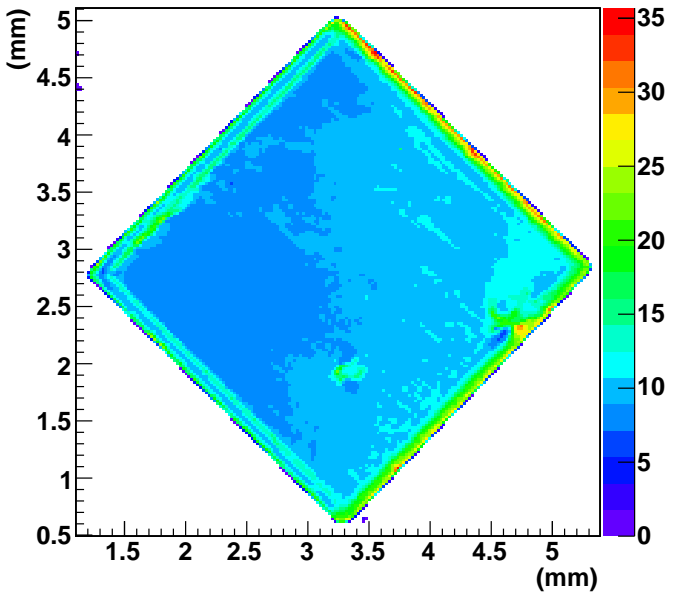

Plate B (vert.) peak centroid ( $\mu \mathrm{r})$

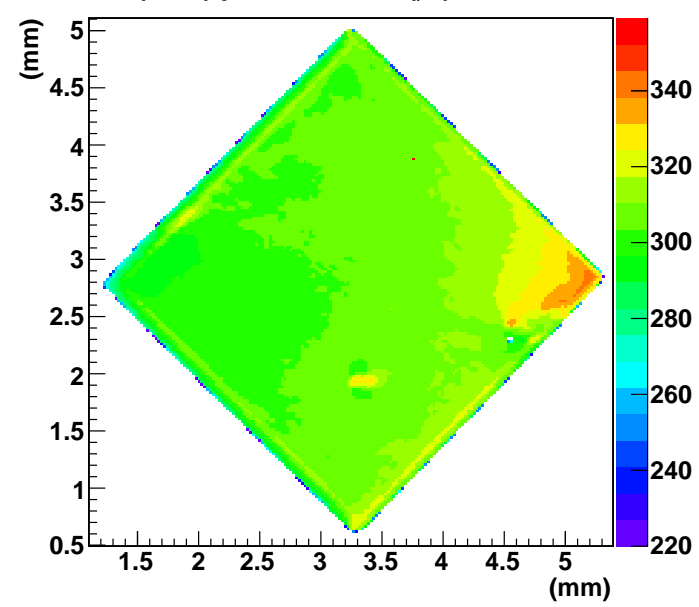

Plate B (hor.) peak r.m.s. width ( $\mu$ r)

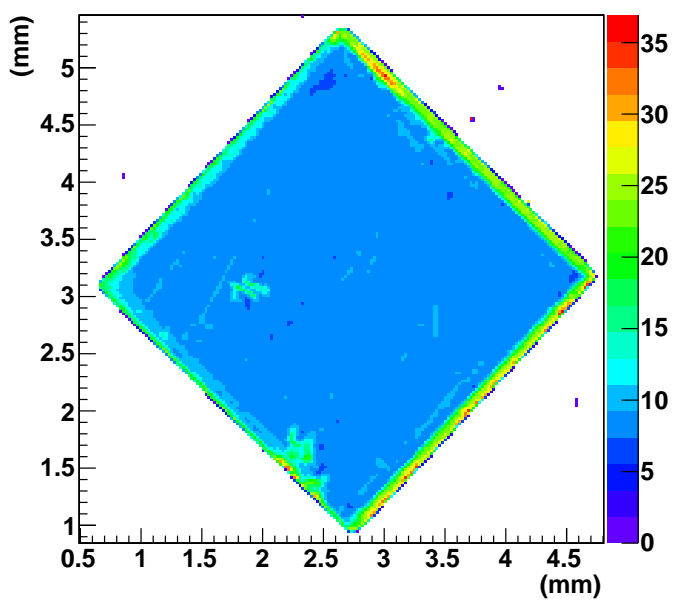

Plate B (hor.) peak centroid ( $\mu \mathrm{r})$

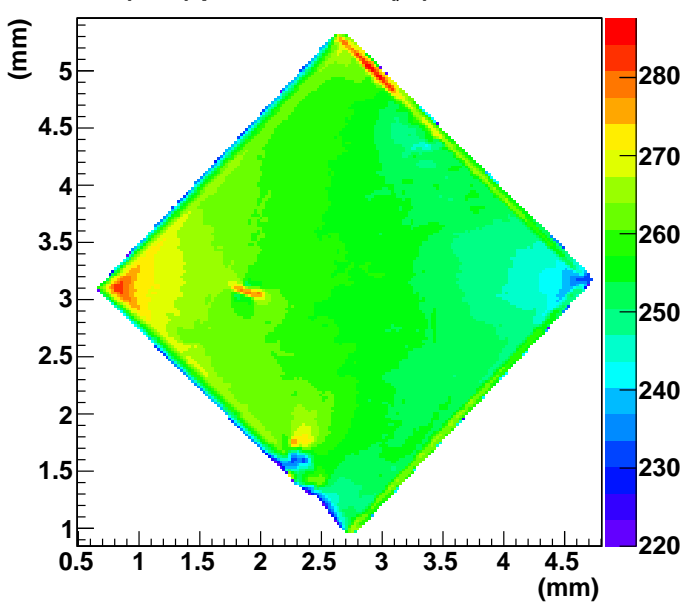

Fig. 2.11: Top row: Plot of local rocking curve width for the non-electron grade "plate" wafer, shown for the vertical and horizontal orientation scans. Note how the inherent rocking curves for most of the diamond surface are comparable to those of the expensive electron-grade diamonds. Bottom row: Plot of the centroid of the local rocking curves examined in the vertical and horizontal scans. This thick, virgin sample clearly holds its normal to the scattering plane uniformly across its surface. 

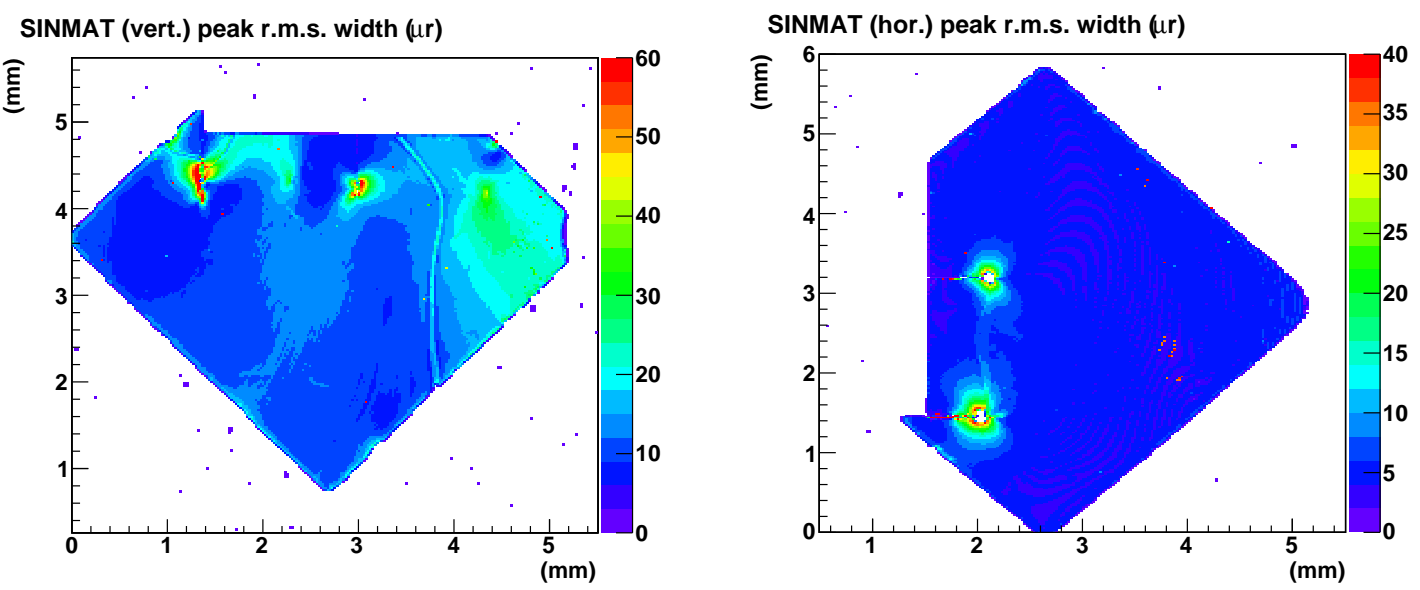

SINMAT (vert.) peak centroid $(\mu \mathrm{r})$
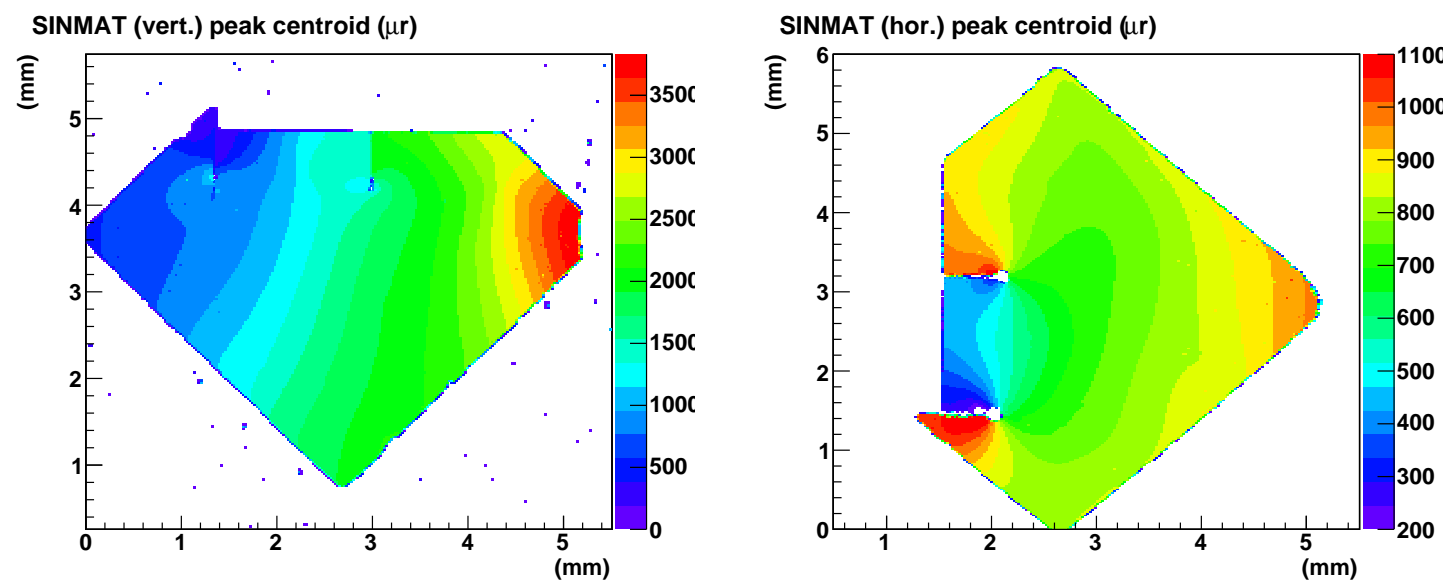

Fig. 2.12: Top row: Plots of local rocking curve width in the two scan orientations for an electron-grade CVD crystal thinned by SINMAT to $9 \mu \mathrm{r}$ using a combination of RCMP and reactive ion etching. Notice that except for spots of significant structural damage, the local crystal mosaic spread is still small. The corresponding distributions of the centroid of the local rocking curves from these scans is shown in the bottom row. They demonstrate that the thinned diamond now flexes well beyond the beamline design tolerance. 

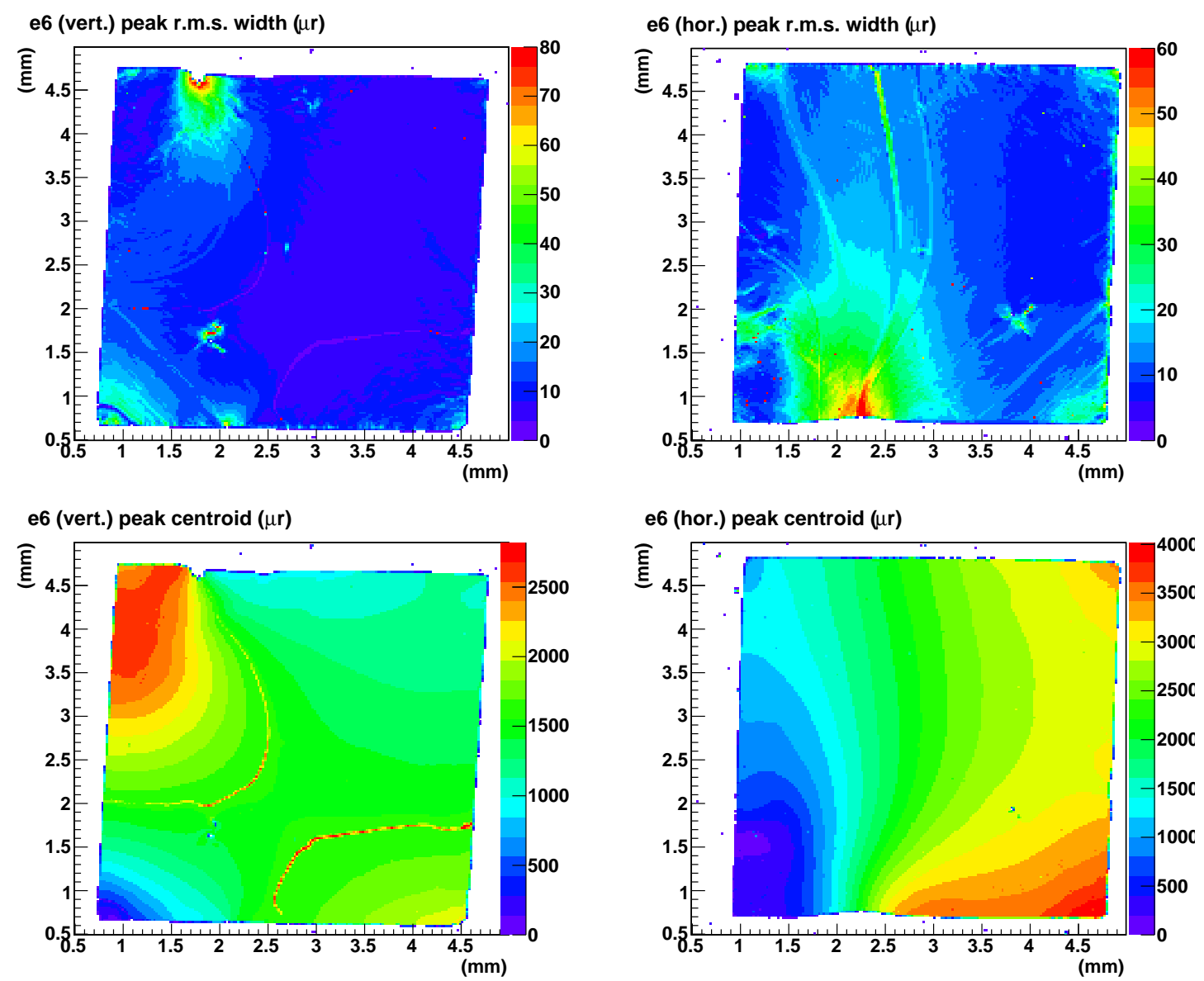

Fig. 2.13: Top row: Plot of the local rocking curve widths for the $10 \mu \mathrm{m}$ Element6 -thinned sample, from scans in the two orientations. The wafer appears to have a small local mosaic spread over most of the surface, with some spots exceeding specification. Bottom row: Corresponding plots of the centroid of the local rocking curves. It is clear that the thin wafer does not hold its form: the normal to the scattering plane travels across the diamond surface and exceeds the design specification. 


\section{Chapter 3}

\section{Tagger Microscope}

\subsection{Introduction}

The introduction to the Hall D photon beamline (Section 2.1) discussed the spectrum of photons from coherent bremsstrahlung $(\mathrm{CB})$, the focus on a peak at $9 \mathrm{GeV}$ and the need to collimate the beam to increase the coherent fraction of the beam. Now we turn our attention to tagging of the generated photons in a way that is efficient, given the beam rate and collimation upstream. The full CB spectrum will be analyzed online by the broadband hodoscope also known as the "fixed array [of tagging counters]". This detector system primarily assists alignment of the diamond radiator, providing feedback on the position of the coherent edges in the spectrum. Though most of this array will essentially be operating in current mode, the high-energy end will be useful for tagging electrons. The band in the spectrum most critical to the GlueX program, the well-polarized photons under the $9 \mathrm{GeV}$ peak, will be tagged by a fine resolution hodoscope. This array, called "tagger microscope" (TM) is designed with a focus on high efficiency tagging with

good energy and time resolution. Figure 3.1 shows the raw and the optimallycollimated photon spectrum and indicates the spectral coverage of the fixed array and the microscope.

The basic requirements for the tagger microscope are as follows: 


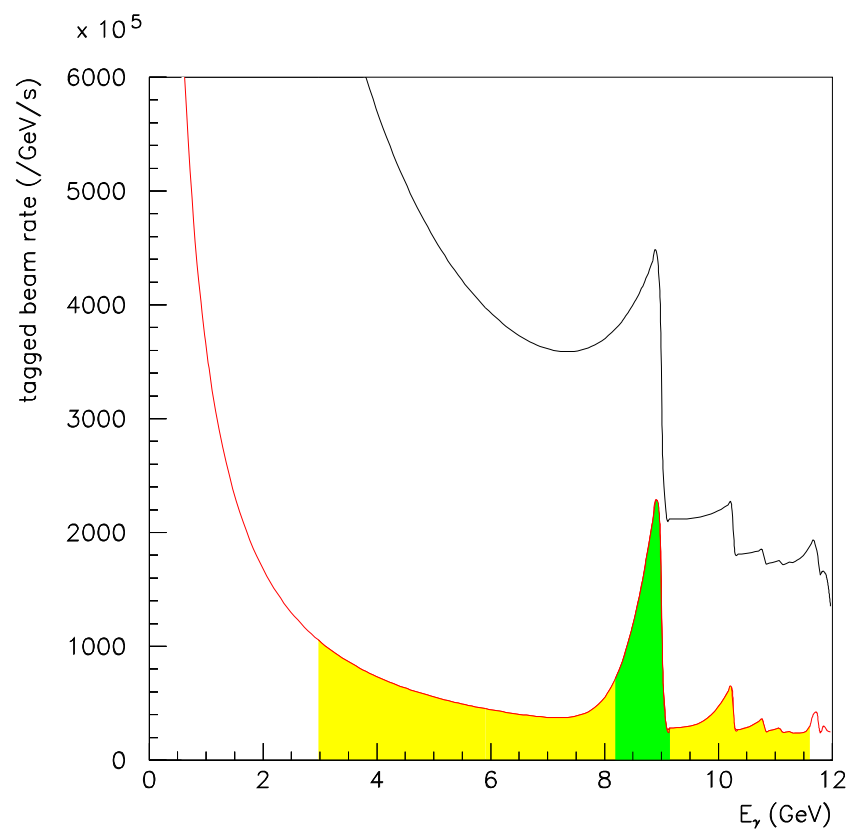

Fig. 3.1: Raw rates on the tagger hodoscopes as a function of photon energy are shown by the upper (black) curve. The post-collimator rate is shown below (red) with the segment counted by the fixed array and microscope highlighted in yellow (light shade) and green (dark shade), respectively.

1. $200 \mathrm{ps}$ timing resolution to ensure identification of the accelerator bunch from a 2 ns-repeating train

2. at least $95 \%$ counting efficiency in every channel

3. $0.1 \%$ r.m.s. energy resolution

These requirements essentially translate toward a need for a scintillating counter detector with high light capture efficiency, narrow sampling of the analyzed beam and low dead time. The technical requirements that derive from these and how they are respected will be discussed in the next few sections.

Conservation of momentum in the bremsstrahlung process implies that a photon's deviation from the beam axis must correspond to the electron's deviation from its nominal trajectory through the tagger. For example, a photon emitted 
at some angle above the $z x$ plane $^{7}$ has its parent electron incident on the focal plane below the spectrometer mid-plane. One can take advantage of this feature to recover some of the tagging efficiency lost to photon collimation by collimating the electrons vertically. This can be done by instrumenting the focal plane with a two-dimensional array of counters. The array is aligned such that the stripe projected by the dispersed electron beam is centered on one of the array's detector rows. Then, readout on all other rows is turned off, effectively rendering the tagger blind to electrons above and below a certain transverse (y) coordinate cut. Figure 3.2 shows a projection of the electron flux on the transverse axis, produced by simulating the particle beam with optimized tagger and quadrupole optics. The fraction of the electrons which radiated a photon that can pass the collimators is highlighted in this histogram. The cut described above essentially means clipping the tails of the distribution to minimize the capture of the electrons without a deliverable photon on target. Figure 3.2 suggests a counter width along the y-axis of $2 \mathrm{~mm}$.

The above argument for controlling the vertical acceptance of the microscope makes a case for the capability to turn off detection in selected fiber channels. However, this does not mean that each fiber must have a dedicated readout digitization channel that will be disabled most of the time. One can reduce the cost of this detector by summing the signals from a single column of fibers (corresponding to one energy bin) and recording only these. The channels turned off simply would not contribute to the summed signal. But it would still be useful to measure the transverse beam intensity distribution shown in Figure 3.2. This would help diagnose the tagger and quadrupole magnet optics and help align the

\footnotetext{
${ }^{7}$ As a reminder, a traditional accelerator coordinate system is used, where $z$ points in the beam direction and $y$ points vertically with the origin located somewhere along the beam path.
} 


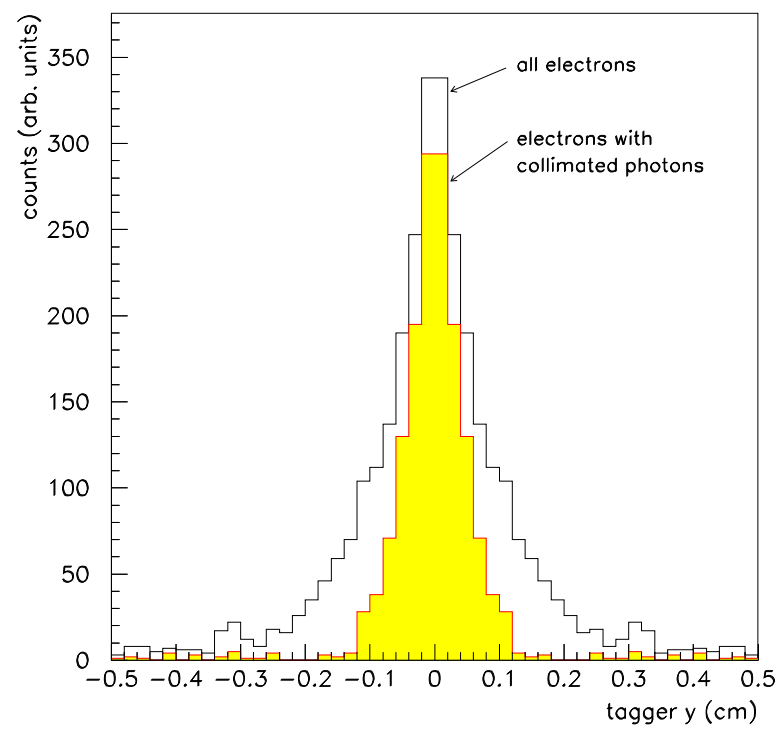

Fig. 3.2: The distribution of electron flux transverse to the dispersed electron beam is shown. The fraction of electrons producing a photon that clears the collimator is highlighted. This figure makes a case for improving tagging efficiency by restricting the tagger acceptance band in the $y$ direction.

electron beam on the desired fiber row. So, the design includes individual fiber readout of occasional fiber columns, sampling the distribution at various points along the focal plane.

Now, let us consider the appropriate focal plane counter width in the direction of tagger dipole dispersion. Here, one has to consider the tagger energy resolution and the counting rate. Figure 3.1 shows the simulated rate as a function of the photon energy. A quick calculation based on these results for the counter at $9 \mathrm{GeV}$ with the required $0.1 \%$ energy resolution, $9 \mathrm{MeV}$, means a counter width of about $30 \mathrm{MeV}$ and a resulting rate of $\sim 14 \mathrm{MHz}$. This is a prohibitively high rate that would lead to a high frequency of pulse overlap, and consequently a low counter efficiency. It turns out that setting the physical counter width to $2 \mathrm{~mm}$ 
$(8 \mathrm{MeV})$ gets this rate down to an acceptable $3.6 \mathrm{MHz}$. The result is a neat design of a focal plane segmented into $2 \mathrm{~mm} \times 2 \mathrm{~mm}$ plaquettes. The obvious choice for fast counters of this cross-section are square scintillating fibers. $2.7 \mathrm{~ns}$ scintillation decay time square fibers with the necessary cladding to isolate the optical channels are commercially available.

Figure 3.3 shows the incoming electrons' view of the fibers with projected beam intensity centered on one fiber row. The diagram also shows one possible plan of fiber array segmentation, in which readout of a 5 column "bundle" is handled by one set of electronics and the left-most column in each bundle may be read out individually, as it is done with 5 columns outlined in blue in the figure.

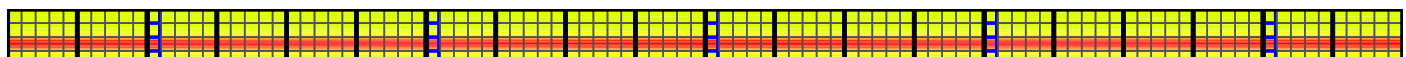
5

Fig. 3.3: This is how the focal plane instrumented with the scintillating fibers looks to the incoming electrons. The red (dark) stripe shows the beam intensity profile once it is centered on a row of fibers. Thin, light gray lines show the segmentation of individual fibers; thick black lines indicate boundaries between fiber bundles. In this segmentation plan a bundle has 25 fibers, which can be read-out by a single 30-channel SiPM amplifier board. Note that other segmentation plans are possible; in all cases, the electronics allows for individual fiber readout in the edge column of any bundle.

For the length in the third axis of these scintillator counters, an optimization was performed of light yield and cross-talk between counters due to electron multiple-scattering, with a final choice of $2 \mathrm{~cm}$. The exit end of this $2 \mathrm{~cm}$-long scintillating fiber is mated with a clear fiber that acts as a waveguide to deliver the scintillation light to the photo-sensor. (Mounting the sensor directly on the back of the scintillator would both be difficult due to the close packing, and would expose the sensors to the electron beam. Later simulations also showed signifi- 
cant levels of neutron radiation in the tagger hall, prompting a design in which the detectors are protected within a shielding enclosure.) The conceptual design of this scheme is shown in Figure 3.4.

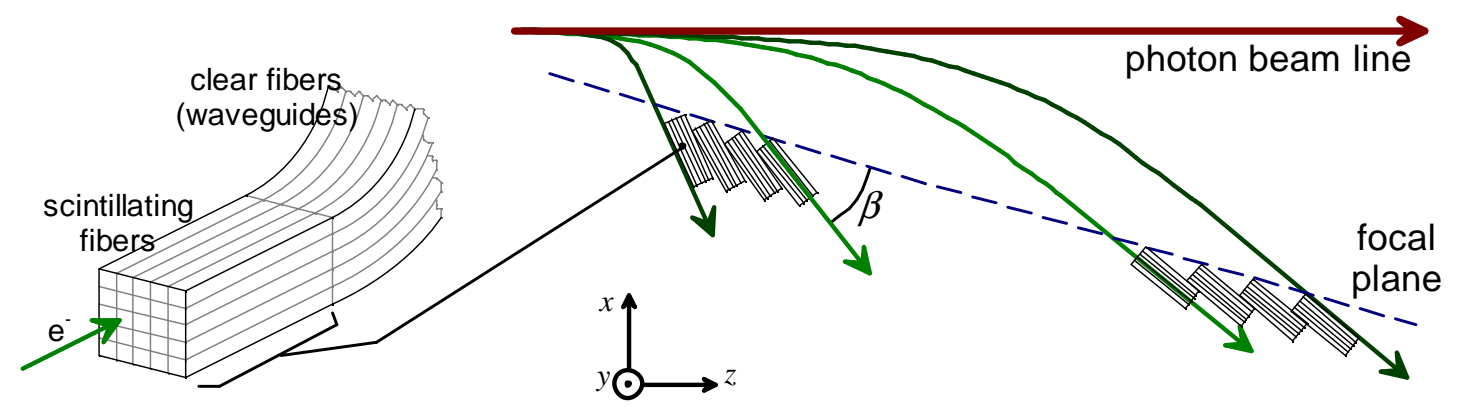

Fig. 3.4: A conceptual design of the focal plane scintillating fiber counter instrumentation (not to scale). Waveguides coupling scintillators to the readout sensors are not shown in the top view but their mating end is shown in the detail sketch in the lower-left.

A new, solid-state photo-sensor device, the silicon photomultiplier (SiPM) was chosen for readout of the scintillating fibers. This design choice was driven by several factors:

1. robust operation in a magnetic field (stray field from the tagger dipole)

2. fast readout that can meet the time resolution requirements of the detector (accelerator beam bunch identification)

3. gain of order $10^{6}$ - consistent with small scintillation flash readout

4. compact size with active area of a few $\mathrm{mm}^{2}$ to match the fiber cross-section

5. no high voltage necessary: typical bias voltage $<100 \mathrm{~V}$

These characteristics make SiPMs far more competitive than the traditional photo-multiplier tubes (PMTs) in this application [27]. Essentially, these are 
multi-pixel photodiodes. Reverse biased to a few volts beyond breakdown threshold, they operate in Geiger mode, producing an avalanche of electrons when a photon is incident on a pixel [7]. Each pixel is a binary device, but the active area of the SiPM contains hundreds to several thousand pixels, offering a considerable dynamic range.

Before forming a detailed design and its optimization it is worth checking whether this scheme is suitable for meeting the requirements of this detector and examine any challenging parameters to drive the R\&D effort. The fastest scintillating fiber found on the market that matches all the other detector specifications was the BCF-20 from Saint-Gobain. Its 2.7 ns decay time can be interpreted as offering an exponential probability density distribution for the emission of any one photon. The uncertainty on the timing in pulses with an average of $\mathrm{N}$ detectable photons is then $2.7 \mathrm{~ns} / \sqrt{N}$. To meet the $200 \mathrm{ps}$ time resolution requirement, it appears that at least 180 photons must be detected on average. Using the canonical $8000 \gamma / \mathrm{MeV}$ and $2 \mathrm{MeV} / \mathrm{cm}$ energy deposition in a $2 \mathrm{~cm}$-long scintillator by an electron leads to $32,000 \mathrm{\gamma} /$ scintillation. With the small capture cone of a scintillating fiber (conservative and simplified assumption of round fiber) of $5.6 \%$ and some worst-case attentuation estimates at fiber junctions and coupling to photosensor leads to an estimated delivery fraction of 4.5\%. Taking the Photonique SSPM-06x SiPM, the closest unit on the market to match the needs of the detector at the time of initial design, we get a scintillator spectrum-weighted detection efficiency of $23 \%$. Folding these capture and detection efficiencies and including the saturation effects of this device leads to 300 fired pixels. ${ }^{8}$ This number is

\footnotetext{
${ }^{8}$ Saturation in this $N_{\mathrm{px}}=1700$ pixel device begins to play a role for a light pulse of a few hundred fired pixels, because some photons will hit already activated pixels. The average number of pixels fired as a function of incident photon number $\left(N_{\gamma}\right)$ is $N_{\mathrm{px}}\left[1-\exp \left(-\eta N_{\gamma} / N_{\mathrm{px}}\right)\right]$, where $\eta$ is the photon detection efficiency, $23 \%$ in this case.
} 
safely above the 180 activated pixel minimum and may be improved upon with better fiber waveguide coupling, reflection on the upstream end of scintillators (to include the backward cone), and by finding a SiPM with a larger active area, detection efficiency and pixel number.

To review, the plan for the tagger microscope detector involves detection of electrons incident on the focal plane with scintillating fibers and conducting the scintillations along a fiber waveguide to the SiPMs located out of the electron beam. The SiPMs are mounted on custom electronics boards instrumented with bias and pre-amplifier circuitry (henceforth "amplifier boards"). To reduce background light for these photo-detectors, this system is meant to be installed in a light-sealed chamber, patching out already amplified pulse signals to the digitizing electronics racks erected outside. The following sections will present a case for an additional set of electronics - control boards - mounted on the outside of the chamber to facilitate control and monitoring of the amplifier boards. The output signals from the detector are planned to be split between an analog to digital converter (ADC) and a leading edge discriminator (LED) feeding into a time to digital converter (TDC) with an optional scaler. Figure 3.5 shows the overall signal flow diagram.

The following sections will cover the details of this system's design, starting with the characterization of the SiPMs, the design of their amplifier circuitry and then the control board designed to interface this detector with the control system.

\subsection{Silicon Photomultipliers}

Silicon Photomultipliers are novel, solid-state, photo-sensors behaving on the principle of Geiger-mode avalanche photo-diodes [7]. The photo-diode is reverse bi- 


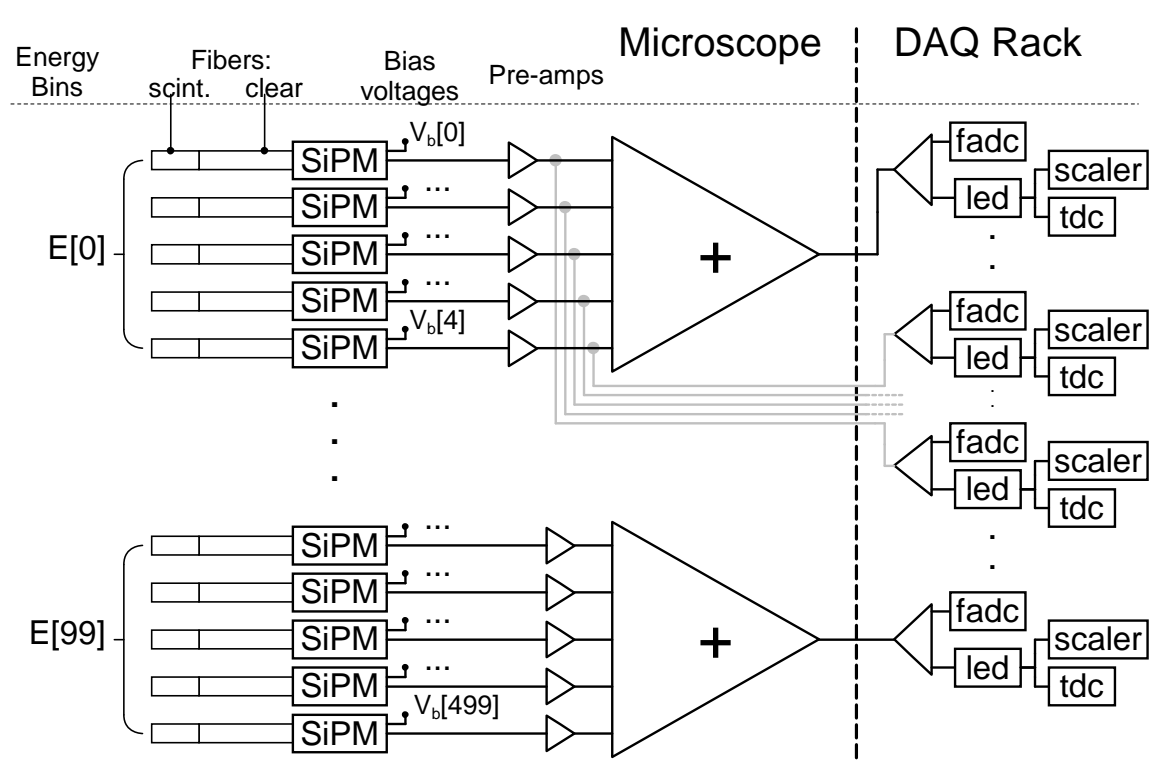

Fig. 3.5: This diagram shows the signal flow sequence, starting from the electron's scintillation in the fiber all the way to readout. The bundles of 5 channels here represent a column of scintillating fibers on the focal plane (one energy bin). Hence, these signals are summed during normal tagger operation. However one sum group per electronics board will also have its individual signals patched out for readout to offer a two-dimensional profile of the dispersed electron beam to help center the beam on a single fiber row. About 5 such columns out of 100 will be read out in this fashion.

ased, charging the effective capacitor of each pixel, $\sim 0.1 \mathrm{pF}$ for the originally studied Photonique SSPM-06 device. Arrival of a photon may trigger an avalanche - a fast discharge of the capacitor. An avalanche may also be triggered by thermal fluctuations in the diode junction, contributing to what is called the "dark rate" of the unit or "dark current". Recharging time is mediated by an effective series resistance in the device and contributes to the dead time of individual pixels.

Perhaps the most important performance parameter of the SiPM with respect to the needs of this detector is the photo-detection efficiency (PDE). This is a combination of the pixels' average quantum efficiency and size of the active area relative to the total. Essentially, it is the probability that an incident photon will 
trigger a response. This parameter is very important to this application because it contributes to the statistical uncertainty of the scintillation timing.

An important task with these new units was the characterization of their performance as a function of their bias voltage and environmental conditions. Performance parameters that need to be tested include: dark rate (current), photodetection efficiency and gain. Additionally, candidate devices must have a fast recovery time with minimal after-pulsing - a constraint to meet the dead time requirements.

SiPMs under test were connected to a simple Photonique-supplied transimpedance amplifier, read out by a digital oscilloscope with an RS-232 connection to a DAQ computer. Studies were performed of SiPM response spectra in the limit of just a few fired pixels. In any regime, the spectrum should approximate a Poisson distribution, since it counts events based on random arrival rate. However, electronic noise and the variation of single-pixel avalanche size contribute to a convolution with variance proportional to the number of fired pixels. Therefore, at low intensity, the peaks are fairly distinct whereas at high intensity, they can no longer be resolved. Resolved photon number peaks offer the ability of separating the sensor's gain and PDE. The spectral separation of peaks indicates the gain per pixel, whereas the Poisson mean compared to the known mean output of a light source offers the detection efficiency. An additional effect is pixel cross-talk: a non-negligible probability that one fired pixel may activate a number of others. This can be taken into account by creating a spectral fit model that couples another Poisson distribution of secondary photon-electrons. Such a model can then resolve the cross-talk parameter in addition to gain and PDE. This spectral fit model can be expressed as follows: 


$$
f(q)=\sum_{p, s}\left(\frac{e^{-\lambda} \lambda^{p}}{p !}\right)\left(\frac{e^{-p \mu}(p \mu)^{s}}{s !}\right)\left(\frac{\exp \left(-\frac{1}{2} \frac{[q-(p+s)]^{2}}{\sigma_{0}^{2}+(p+s) \sigma_{1}^{2}}\right)}{\sqrt{2 \pi\left(\sigma_{0}^{2}+(p+s) \sigma_{1}^{2}\right)}}\right)
$$

where,

$$
\begin{array}{ll}
q=\frac{x-x_{0}}{g} \quad \begin{array}{l}
\text { is the normalization to the pixel count, with gain } g \text { equaling } \\
\text { the distance between peaks in } V s ;
\end{array} \\
\quad \text { stand for the actual pulse integral and that for an average } \\
\quad \text { zero-pixel signal (i.e. center of the pedestal peak); } \\
\quad \begin{array}{l}
\text { are primary Poisson parameters representing the primary } \\
\text { fired pixels and the average pixel count; }
\end{array} \\
\quad \text { are the secondary Poisson factors representing the number } \\
\text { of secondary pixels per real hit from the set of } p \text { hits and } \\
\text { corresponding average secondary count (cross-talk rate); } \\
\text { are noise parameters modeling the overall smearing and pixel- } \\
\text { cumulative convolution, respectively. }
\end{array}
$$

Thus, the gain $(g)$ and cross-talk rate $(\mu)$ are determined immediately from this fit. The mean primary pixel count $\lambda$ divided by the average number of incident photons on the SiPM window gives the PDE. Figure 3.6 shows a sample spectrum from the Photonique SSPM-06x illuminated by small LED flashes. Using these techniques, various SiPM devices were characterized to determine their gain, PDE and dark rate as a function of temperature and bias voltage.

The devices were set to a controlled temperature and bias voltage and then left in the dark, with data acquisition system triggering randomly to capture the noise and dark rate spectrum. This procedure was repeated with a light pulser of calibrated intensity and light-coupling fraction to the SiPM. An event set was now recorded triggered by the pulser. The first procedure yields the dark rate and the second offers the PDE. Both procedures must yield a consistent result for the gain. Data is then acquired at a different configuration of temperature and bias 


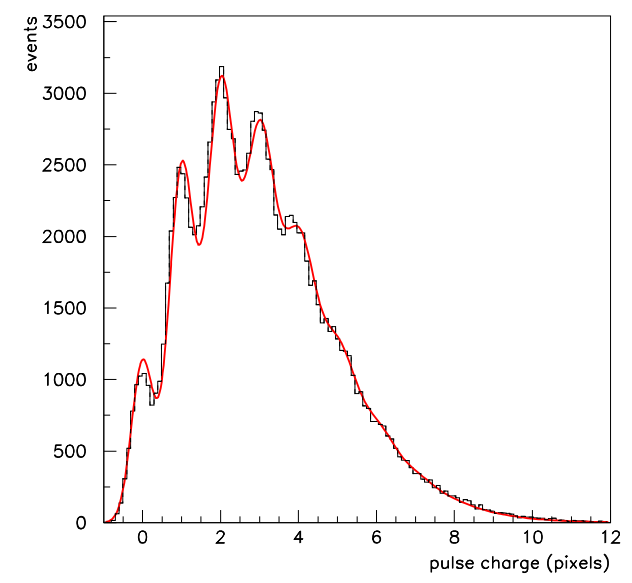

Fig. 3.6: A multi-Poisson model fit to a sample spectrum from a series of pulses collected from Photonique SSPM-06x SiPM illuminated by small flashes of light from and LED.

voltage until the desired configuration space is explored.

Figure 3.7 shows the results of a study of the Photonique SSPM-06x device. The temperature dependence result indicates that precise temperature stabilization will not be important but a reasonable temperature control must be implemented for this detector. Densely packed SiPM readout electronics in a closed (light-tight) space can easily drive the environment above $30^{\circ} \mathrm{C}$ causing a significant loss in PDE. As expected, the sensitivity to bias voltage is more significant. Added to the fact that optimal operating voltage in individual devices can vary on the scale of a volt leads to a conclusion that the detector needs bias voltage control separately for each SiPM. This sensitivity may also be useful for equalizing the pulse amplitudes across the detector's channels and compensating for those with poor optical coupling. In all cases, it appears that the dark rate is negligible for this device. The calculated rate of pileup signals that exceed any reasonable threshold is negligibly low. 

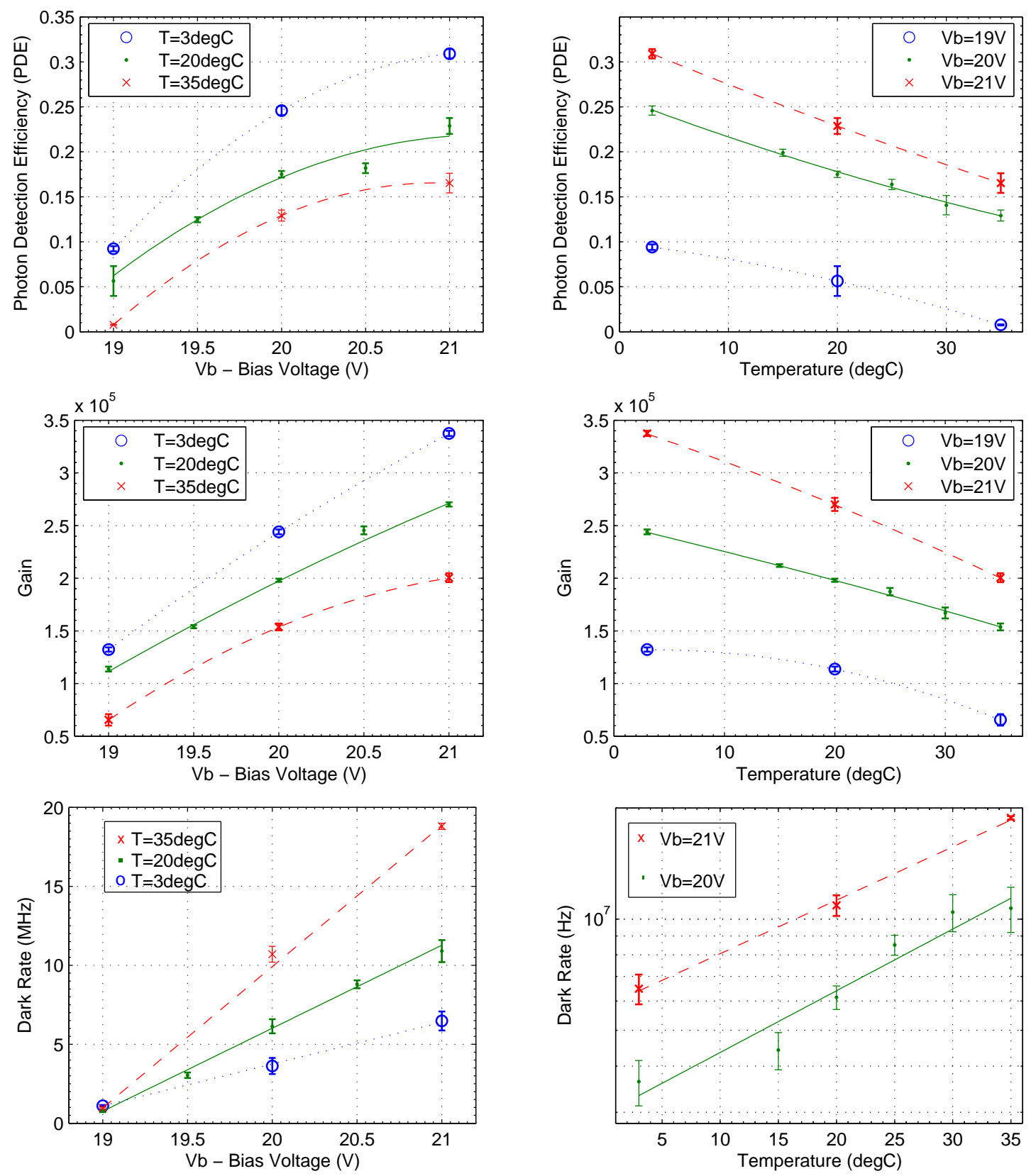

Fig. 3.7: Sensitivity of SiPM photo-detection efficiency, gain and dark rate to the bias voltage and temperature. 


\subsection{Amplifier Electronics}

\subsubsection{Requirements}

Since the SiPM may be regarded as a weak current source, a trans-impedance amplifier is necessary to condition the signal into a form readable by the digitizing electronics. The detector is intended to operate with a leading edge discriminator (LED) as well as a flash analog-to-digital converter (fADC): JLab's 12-bit fADC250. The fADC's parameters relevant to the signal source design are shown in Table 3.1.

Table 3.1: Jefferson Lab's VME64x Flash ADC Module (fADC250) specifications constraining SiPM amplifier design.

\begin{tabular}{ll}
\hline Input impedance & $50 \Omega$ \\
Input range & negative polarity $0.5 \mathrm{~V}, 1 \mathrm{~V}, 2 \mathrm{~V}$ modes (jumper-selected) \\
Sampling rate & $250 \mathrm{MSPS}$ \\
Resolution & 12 -bit \\
\hline
\end{tabular}

Table 3.2 lists the formal requirements of the amplifier design set forth to meet the readout specifications and overall detector requirements. Design stage simulation of a circuit complex enough to meet all the requirements is essential. Thus, we wrote a solver based on the linear equations of passive circuit components and a linearization of transistor equations from the Gummel-Poon model. The program first solves for the quiescent points of the transistors and then performs a linear expansion of the transistor response around these points. After this tuning, the circuit's response is determined for each input frequency on every net in the circuit. A modeled input signal is thus simulated by feeding in the components of its Fourier series. 
Table 3.2: Detailing of the performance requirements for the SiPM preamplifiers and how they may be translated into design features or constraints. More general additional requirements include: 1. summation of channels in groups of five with individual channel readout available on select columns, 2. minimum power consumption within the constraints of the specifications below.

\begin{tabular}{|c|c|}
\hline Specification & Performance Requirement \\
\hline $200 \mathrm{ps}$ & $\begin{array}{l}\text { fast-rising edge to ensure consistent time pick-off; } \\
\text { sufficient signal width for time walk correction }\end{array}$ \\
\hline $95 \%$ efficiency & $\begin{array}{l}\text { fall time less than } \sim 20 \mathrm{~ns} \text { (based on simple discrim- } \\
\text { inator models) }\end{array}$ \\
\hline diagnostic mode & $\begin{array}{l}\text { high gain mode sufficient to resolve single photons } \\
\text { from an external light pulser to measure detection } \\
\text { efficiency and gain of SiPMs }\end{array}$ \\
\hline $\begin{array}{l}\text { channel-to-channel con- } \\
\text { sistency }\end{array}$ & $\begin{array}{l}\text { less than } 15 \% \text { gain variation as a function of transis- } \\
\text { tor } \beta \text { parameters }\end{array}$ \\
\hline fADC range & $\begin{array}{l}\text { pulse amplitudes consistent with either: } \\
-0.5 \mathrm{~V},-1.0 \mathrm{~V} \text {, or }-2.0 \mathrm{~V} \text { modes }\end{array}$ \\
\hline $50 \Omega$ output impedance & impedance matching on long traces in output \\
\hline
\end{tabular}

\subsubsection{Individual and Summed Readout}

The default readout mode of the SiPM pre-amplifier output involves taking the sum of SiPM channels representing a single energy bin group. To remind the reader, the SiPMs are grouped such that their corresponding scintillating fibers are arranged in a column transverse to the dispersed electron beam. All rows but the one on which the beam is centered would ordinarily be turned off by zeroing the bias on their SiPMs. However, as mentioned earlier, for alignment of the scintillator array with respect to the electron beam and for centering a desired scintillating fiber row on the beam, some two-dimensional information about the electron beam projection on the scintillator array is necessary. This is why about 
five, well-spaced columns in the scintillator array must have its individual fibers read out separately.

To meet this requirement, the circuit's design includes two stages: a singlechannel amplifier with buffer for readout and a circuit summing the outputs of five single-channel amplifiers. Because there will be five times as many instances of the first stage, but it will be read out separately least often, and because the data critical to high speed experimental data-taking comes from the summer, most of the complexity was pushed into the summing stage. Specifically, gain mode selection and extra buffering were designed into the summer.

\subsubsection{Gain}

The gain settings of the amplifier must be designed in a way to ensure that the signals are within the dynamic range of the flash ADC and are not sensitive to the voltage resolution of the device. These soft constraints help define the standard operating gain and the high gain mode. (The latter must be high enough so that signals corresponding to just a few photons can be resolved and that their spectrum would not be significantly affected by the digitization noise.)

Given that the SiPM signal comes in the form of charge injection, the shape of the input signal must be taken into account to calculate the amplitude and set the gain accordingly. As an initial guess in the beginning of the design, an approximation was made, modeling the input and output signals in time with triangular shapes without bandwidth-induced distortion. This estimate (Table 3.3) suggests a normal-running mode of $500 \Omega$ trans-impedance gain. About an order of magnitude higher gain was desired to ensure good spectral peak separation in photon counting mode. Another issue during the design stage for this detector is 
Table 3.3: Estimate for the necessary transimpedance gain of the SiPM preamplifier at the start of the circuit's design. This estimate models the input and output signals as triangles without change of shape due to bandwidth limitations.

\begin{tabular}{l|r|l}
\hline \hline Parameter & Value & Units \\
\hline SiPM gain & $2 \times 10^{5}$ & - \\
Expected photo-electrons & 300 & - \\
Resulting charge collected & 9.6 & $\mathrm{pC}$ \\
Input signal FWHM & 15 & $\mathrm{~ns}$ \\
Resulting peak current & 0.65 & $\mathrm{~mA}$ \\
TDC/ADC signal split factor & 0.5 & - \\
Dynamic range occupation of expected signal & 30 & $\%$ \\
Intended fADC250 range & $-1-0$ & $\mathrm{~V}$ \\
Result: necessary gain in pre-amp & 500 & $\Omega$ \\
\hline
\end{tabular}

that the budgeted fADC unit had only 10-bit resolution, demanding higher gain. So the goal was to reach approximately $5 \mathrm{k} \Omega$.

\subsubsection{Frequency Response and Bandwidth}

Deriving from the detector's general requirements and some general rules of RF circuit design, we can set forth the following guidelines for designing the frequency response of this circuit:

1. input and output must be AC-coupled

2. the $250 \mathrm{MHz}$ sampling rate of the fADC250 offers a Nyquist frequency upperbound of $125 \mathrm{MHz}$

3. optimized high-frequency response (within the above bounds) to achieve a narrow pulse (fast rise time/low dead time requirement)

4. frequency band lower-bound tuned to minimize the swing after the pulse peak to minimize baseline shifts 
5. minimal coupling of noise: low response in common noise bands and good isolation to avoid unwanted feedback and cross-talk

6. stability: inductance minimized to avoid resonances

Much of the effort in the design of this circuit went into fulfilling point 3 listed above. The points in the circuit that can be modeled as current injectors, the amplifier and the summing circuit inputs, had to be of low impedance to prevent large RC integration time. This was solved by connecting these inputs to the emitters of the input stage transistors, where the impedance has the approximate form:

$$
Z_{E} \sim \frac{1}{g_{m}}+\frac{Z_{B}}{\beta_{0}}
$$

where $g_{m}$ is the transconductance of the transistor, proportional to quiescent collector current and $Z_{B}$ represents the source impedance of the transistor base biasing circuit. With a low impedance path through the base at high frequency and biasing arranged such that the collector DC current is large, the impedance of input on the emitter can be minimized. Simulations showed that this design approach with about $2 \mathrm{~mA}$ quiescent collector current would yield $13.5 \Omega$ and $27.3 \Omega$ for the amplifier and summing circuit input impedance, respectively.

The complete circuit is shown in Figure 3.8 and includes a simplified description of the different circuit stages' roles. Note the FET switch in the summing circuit: this effectively controls the resistance on the (transistor $m$ ) emitter at AC, which is roughly proportional to the gain of this stage.

Figure 3.9 shows the simulated amplifier response after the circuit's design was optimized. First, an approximation to the input signal was made in the form 


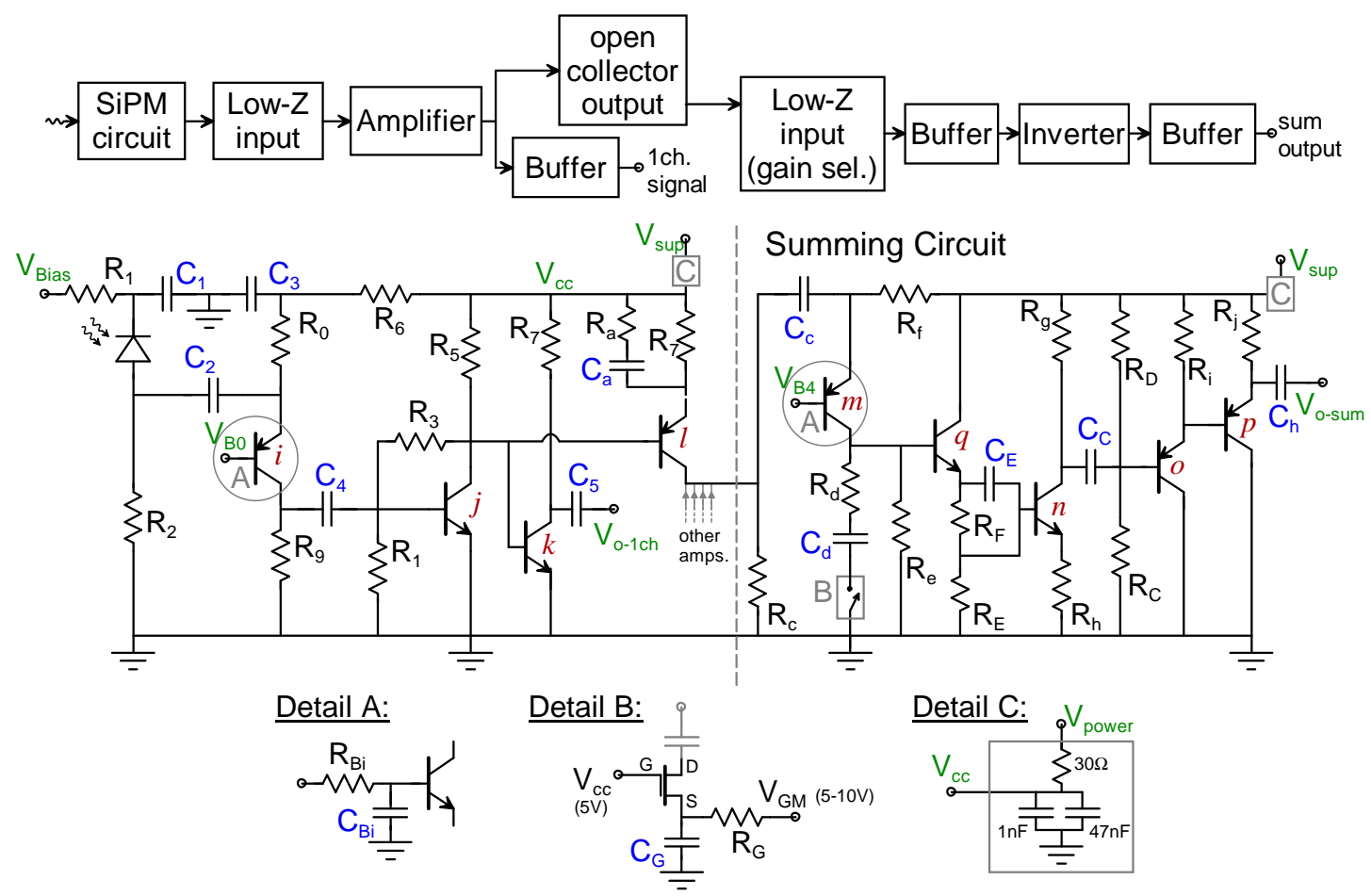

Fig. 3.8: Schematic of the SiPM bias, amplifier and summing circuit. A functional diagram of this electronics is given above the schematic, roughly illustrating the function of the transistor stages. Note that five instances of the circuit to the left of the vertical dashed line (representing the initial pre-amplifier and individual channel buffer) with its SiPM connect to a single summing circuit on the right of the dashed line. For simplicity, just one pre-amplifier is drawn connected to the summer.

of a Gaussian with a width derived from the following simple test. A SiPM was driven with a light pulser barely over threshold to produce a fast pulse. The SiPM was read out directly, without an amplifier. The signal was deconvolved using the expected effects of the cable and oscilloscope frequency bandwidths. In practice, any fast input signal suffices for the simulation, since the light signals incident on the future detector's photo-sensors would be dominated by the 2.7 ns characteristic time spread of the BCF-20 scintillator. The simulation of the optimized design shows a modest integration time that is within specification of the needed pulse 

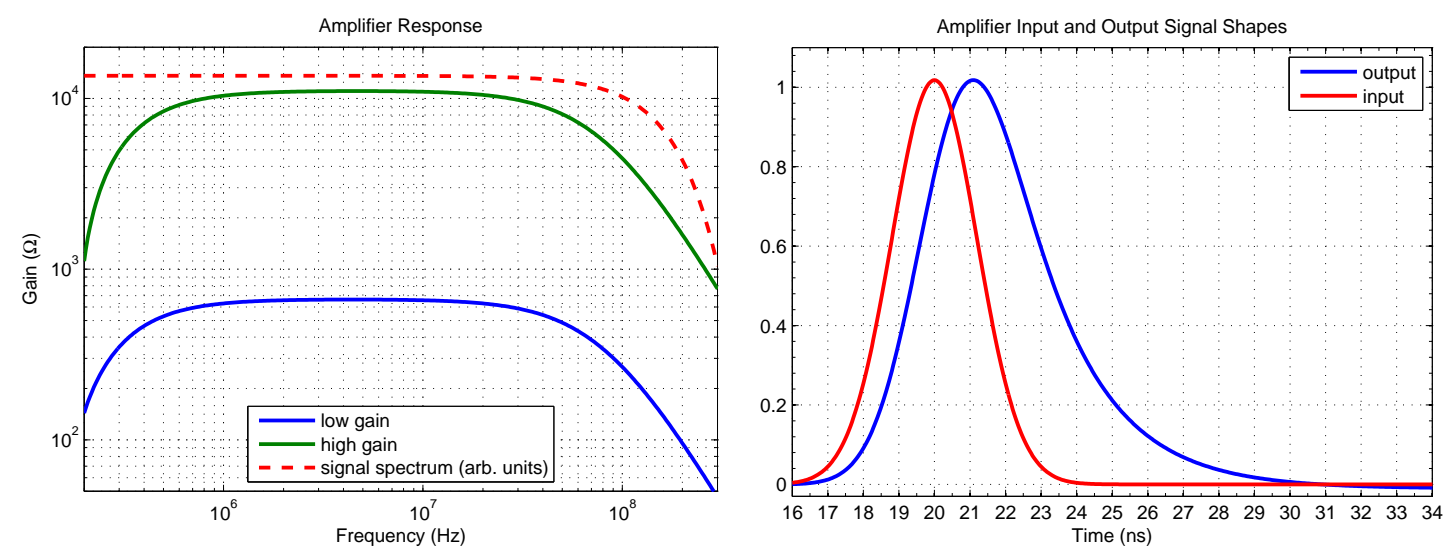

Fig. 3.9: The plots show the simulated amplifier response in frequency and time domains. An approximation to the input signal shape and spectrum is given based on some un-amplified recorded signals from the SiPM deconvolved based on expected oscilloscope and cable bandwidth effects.

decay time.

Figure 3.10 shows the frequency response of the circuit in the low-gain mode as it was designed and what was measured in the first manufactured prototype. The measurement was performed by injecting a small sinusoidal current directly into the input stage of the amplifier and measuring the response. The measurement shows significant deviation from the designed response: a broad enhancement centered around $2 \mathrm{MHz}$ and several resonances on the scale of $100 \mathrm{MHz}$. A subsequent iteration on the circuit's layout has solved these problems, improving the design of traces that may have added significant parasitic inductance. (Models of the created oscillators showed resonances compatible with those measured in this circuit.) The higher gain at low frequency was later understood to be the result of using a lower capacitor value for $C_{d}$ than designed, resulting in an effective higher impedance at lower frequencies.

Cross talk at the level of about $15 \%$ was also found in this circuit. The later iteration reduced the packing density of amplifiers on the board, improved 


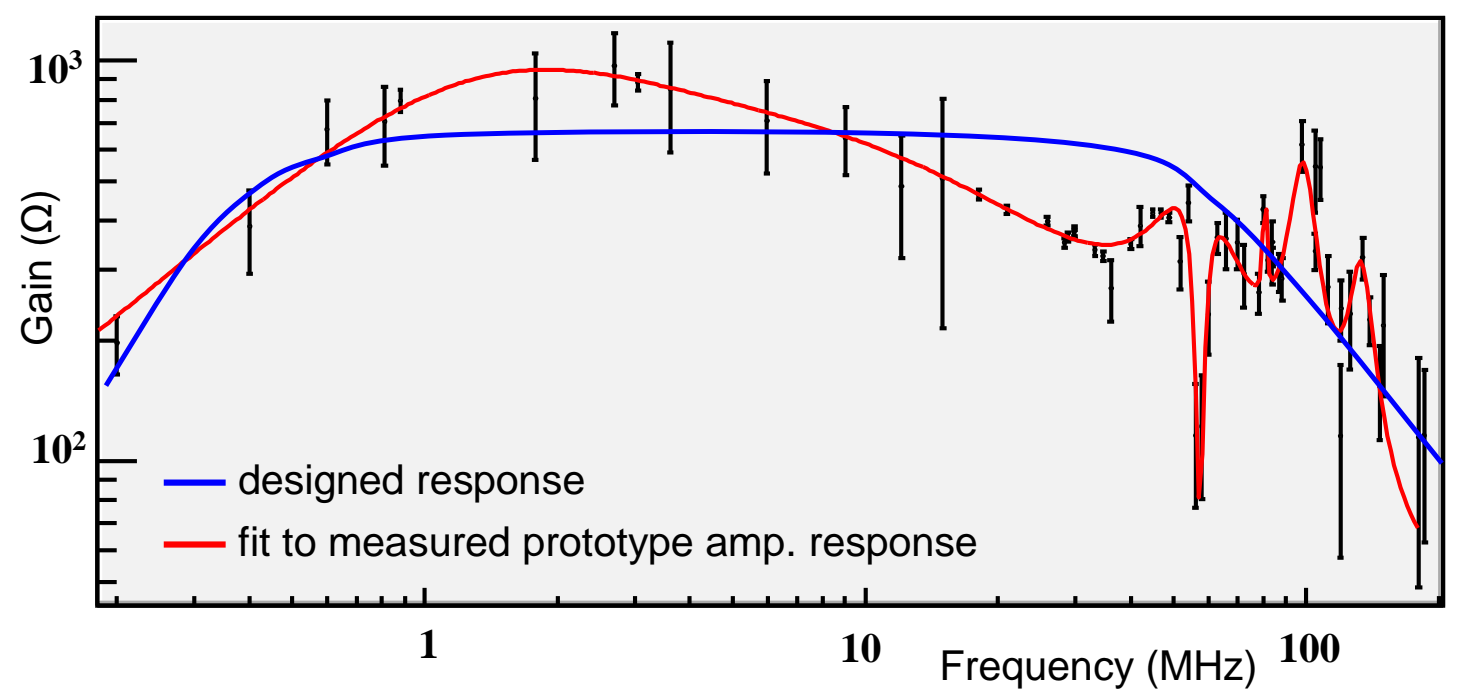

Fig. 3.10: The design frequency response is compared to the measured in the first manufactured prototype. The time domain pulses had a longer fall time, corresponding with the low frequency enhancement and showed significant ringing compatible with the large resonance near $100 \mathrm{MHz}$.

ground and power connections to reduce inductance, isolated the power supply circuit among the amplifiers and included more ground pours to bring cross-talk to negligible levels. A further improvement planned for the circuit is a compensation for rate-dependent SiPM bias voltage. Presently, the pulse rate on the scale of the running regime in the tagger hall will cause enough average current flowing through the SiPM to produce a significant voltage drop across $R_{1}$, requiring a correction by the operator. A current-sensitive voltage-compensating circuit has been designed and will be prototyped and incorporated in the future.

\subsection{Control Electronics}

\subsubsection{Motivation}

The following issues motivated the designers to consider a separate set of electronics to oversee the functionality of the amplifier board. 
1. Bias voltage control:

(a) The breakdown voltage of the SiPMs varies significantly from one unit to another. Offerings from different firms ranged from $20 \mathrm{~V}$ to over $70 \mathrm{~V}$, and device to device variations were of several volts. Light coupling quality may vary as well. This situation required the bias voltage for each SiPM in the detector to be calibrated separately.

(b) The photo-detection efficiency and gain of SiPMs exhibit strong temperature dependence. Online adjustment of their bias voltages to compensate is therefore important.

2. Amplifier gain mode selection: as per the amplifier board design, switching between unity and large gain modes in the summing circuit is necessary in order to switch between normal running and photon-counting-based diagnostics regime.

3. Monitoring of amplifier board health:

(a) Temperature: Monitoring the amplifier board temperature is prudent in light of the SiPMs' sensitivity to this parameter, the amplifiers' significant power output and their placement in a light-sealed chamber with potential difficulties in heat exchange.

(b) Voltage: There are three DC voltage levels on the amplifier board that are useful to monitor. The supply voltage effects the amplification and dynamic range of the signal: feedback on this value is useful to the operator for online adjustment. The other two: base voltages for the input stages of the amplifiers' main circuit and summer are sensitive parameters that cannot be adjusted remotely and would signal the 
need to replace the board in the event that they no longer conform to specifications.

Note that this detector component, like most others, sits in areas of dangerous radiation levels during experimental operation. All monitoring and control must be performed remotely from the Counting House. This implies a requirement of a communication bus between this system and the operator control computers: a distance on the order of a hundred meters. The ability to multiplex between different control/monitoring channels or groups thereof imposes another requirement on the bus standard or encoding protocol used in communication with this system.

\subsubsection{Implementation}

This layer of electronics was implemented as a separate set of boards paired with one or several amplifier boards. One of the reasons for this was to isolate the digital signals in the control circuitry from the analog signals of the amplifier board. Another reason was to enable attachment of this hardware to the outside of the light-sealed photo-detector chamber in order to relieve the challenges and costs of patching cables through light-tight interfaces.

The requirement of implementing control from a distance of hundreds of meters can be met with several existing communication buses. Choosing a modern, commodity technology was also an important consideration. Though potentially more complex to implement than other standards, CAT-5-based Ethernet communication seemed the most attractive, as it is a commodity, long-range system naturally-multiplexing and commonly used in experimental areas. For simplicity, the design called for simple Ethernet packets with each control board-operated 
segment of channels being addressed via the MAC address. However, the designers wanted to avoid having to catalog the MAC address of each controller and cross-reference it with the range of energy bins it serves. Instead, the design allows for each control board to acquire a one-byte "geographic address" hard-coded in the slot into which the board is seated and stamp its communications with this address. This address can be a simple energy bin group number, since the fiber waveguide patching scheme already defines the mapping from energy bins to the electronics sets. For example, the 100 energy bins (500 SiPM channels) divided into 20 segments would be instrumented with electronics slots with addresses 0x00 through 0x13, thus the address pattern would follow the energy bin order. The software would then automatically build a table, cross-referencing the MAC address of the communicating board with the geographic address it provides and expose to the user just the focal plane segment he or she is controlling.

The group searched for commodity integrated circuits that can bias a large class of SiPMs: known units required bias of over $70 \mathrm{~V}$. Preference was made for units with many channels in order to handle large groups of SiPMs at once. The design settled on the Analog Devices AD5535. This is a 32-channel DAC ranging to up to $200 \mathrm{~V}$ output and more than sufficient current for the expected steady-state operation of the detectors.

The lack of voltage read-back on these units motivated a design in which one DAC channel is dedicated as a voltage-setting test line. In other words, this line would not be connected to any SiPM and will rather be set to a known value and then sampled by the ADC unit involved in electronics health monitoring (described below). Another line must be dedicated to amplifier gain-mode selection, setting the corresponding FET switch gates on the amplifier board. What remain are 30 
Table 3.4: Allocation of the ADC channels for monitoring critical sytem voltages.

\begin{tabular}{|c|c|c|}
\hline Ch. & Line/Net & Description \\
\hline 0 & VHEALTH & $\begin{array}{l}\text { composite health indicator: monitors the output of a volt- } \\
\text { age divider set between }+5 \mathrm{~V} \text { and }-5 \mathrm{~V} \text {; Nominal value: } \\
2.51 \mathrm{~V}\end{array}$ \\
\hline 1 & CATHODE & $\begin{array}{l}\text { output of temperature-measurement diode the DAC; typi- } \\
\text { cal value: } 4.35 \mathrm{~V} \text { at } 25^{\circ} \text { with dependence of }-2.20 \mathrm{mV} /{ }^{\circ} \mathrm{C} \text {. }\end{array}$ \\
\hline 2 & $+3.3 \mathrm{~V}$ & $\begin{array}{l}\text { monitors the }+3.3 \mathrm{~V} \text { power line controlled by device VR1 } \\
\text { on board }\end{array}$ \\
\hline 3 & $+5 \mathrm{~V}$ & monitors the $+5 \mathrm{~V}$ power line i.e. external power supply \\
\hline 4 & ADC_EXT1 & $\begin{array}{l}\text { measures the amplifier board input stage transistor base } \\
\text { voltage }\end{array}$ \\
\hline 5 & $+1.2 \mathrm{~V}$ & $\begin{array}{l}\text { monitors the }+1.2 \mathrm{~V} \text { power line controlled by device VR3 } \\
\text { on board }\end{array}$ \\
\hline 6 & ADC_EXT2 & $\begin{array}{l}\text { measures the voltage on a pin of the thermistor on the } \\
\text { amplifier board }\end{array}$ \\
\hline 7 & DACHEALTH & $\begin{array}{l}\text { monitors the output channel } 31 \text { of the DAC rescaled by } \\
0.0249 \text { to remain within the ADC range over the full } 200 \mathrm{~V} \\
\text { range of the DAC }\end{array}$ \\
\hline
\end{tabular}

channels, suggesting a natural grouping of SiPM channels. With five rows in the two-dimensional scintillator array, up to six columns (or energy bins) are possible in each fiber group.

For monitoring the health of the control and amplifier boards, an 8-channel Analog Devices AD7928 ADC and an Analog Devices AD7314 temperature sensor have been selected. The latter measures the temperature on the control board while a channel of the ADC is dedicated to measuring the voltage across a thermistor on the amplifier board to learn the temperature there. The remaining seven channels of the ADC are used to sample key DC voltages on the amplifier board and the control board (details in Table 3.4). These two devices are a convenient pair to employ as they follow the same SPI-like bus protocol, allowing multiplexing between these devices on the same bus. 
The Field-Programmable Gate Array (FPGA) was the technology selected for the central controller. The FPGA would order voltage and temperature readings, drive the encoding of the DAC and interface with the controlling computer by reading and writing packets through the Ethernet Controller (EC). The Xilinx Spartan 3A XC3S50A was chosen for this application.

\subsubsection{Implementation details of the FPGA}

The programming of FPGA gates occurs at startup based on a configuration (firmware) loaded into some non-volatile memory. FPGAs with on-board nonvolatile memory did exist at the time of the design but were avoided as it seemed to be a fairly novel technology. Decoupling programming and execution ICs also seemed to be the easier design approach. Thus, the traditional method was used: EEPROM-based loading using the Xilinx XCF01SVO20C EEPROM. The programming stream itself is synthesized on a computer based on a set of instruction written usually in either VHDL (Very-high-speed integrated circuits Hardware Description Language) or Verilog. Rather than the model of computer programming, in which the sequence of execution steps are outlined, FPGA programming takes the hardware to be a parallel set of state machines, the behavior of which must be defined in a complete way by the code. Thus, the programming of this device involved the coding of modules inside the FPGA, their simulation with respect to input and output signals and examination of the gates instantiated in and inferred from the code.

For simplicity, a single digital clock was desired for the entire board. Since the temperature sensor had the most restrictive bus speed of $10 \mathrm{MHz}$, a conservative setting of $5 \mathrm{MHz}$ was used for most of the operations. However the Ethernet 
Controller application notes advised a $20 \mathrm{MHz}$ external clock. The Intel Bus protocol between the EC and the FPGA also required a timing structure more easily organized with a clock of around $20 \mathrm{MHz}$. Thus a FOX FXO-HC53 HCMOS $20 \mathrm{MHz}$ clock IC was used to drive the FPGA and the EC with the FPGA subdividing this clock to create a $5 \mathrm{MHz}$ signal for all other board components and most of its internal logic.

The FPGA firmware is designed based on a set of modules that perform discrete tasks in the execution cycle of the control system: idling (waiting), reading of new instruction packets, querying the monitoring system, programming the DAC and writing new packets to respond to the controlling PC using the EC. The modules are selectively activated using a 3-bit state register. A module deactivates itself and passes control to its successor by changing the register appropriately when finished. The overall organization is illustrated in Figure 3.11. The single "state-less" module is the transceiver, which serves as an interface between FPGA modules and the EC. Aside from being the protocol bridge, this module separates the domains of the two clocks mentioned above. FPGA's modules have internal subunits that are able to communicate to external ICs as necessary. For example, the proprietary DAC bus protocol is coded into a subunit of the "Programmer" module while the "Querier" abstracts the communication to the SPI-bus-based $\mathrm{ADC}$ and temperature sensor.

\subsubsection{Communication Cycle}

Figure 3.12 shows the communication cycle between a control board on the TM and the controlling computer (PC). The packet types shown are specified in the second byte of the Ethernet packet payload, after the first "geographic address" 


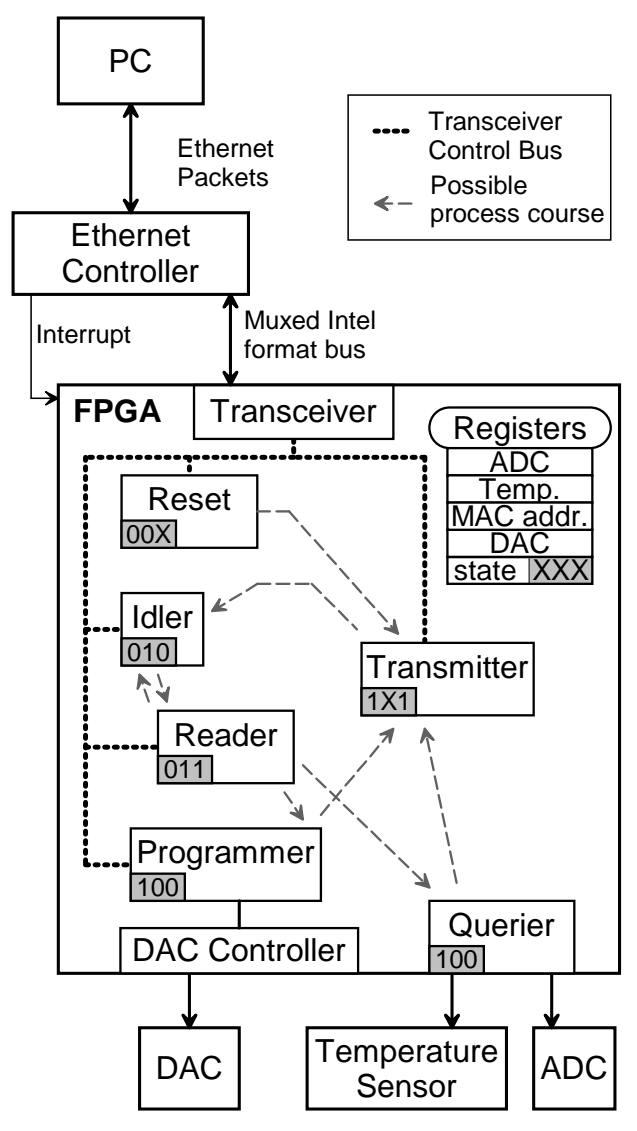

Fig. 3.11: This diagram illustrates the communication scheme between the control board components and some internal FPGA modules. Specifically, it shows how communication on the internal FPGA "transceiver control bus" is orchestrated with a state register. The register codes serve as "chip enable" bits to prevent multiple drivers from writing on the bus. Modules pass control to each other by setting this code after they are done to create the proper chain of events to enable Ethernet communication with the master-computer.

byte.

The diagram outlines the address self-cataloging scheme described above. The boards wake up at power-on or reset with a standard configuration supplied in the EEPROM-recorded firmware, and configure the Ethernet communication settings using the protocol's auto-negotiation. Once this is complete, the boards 


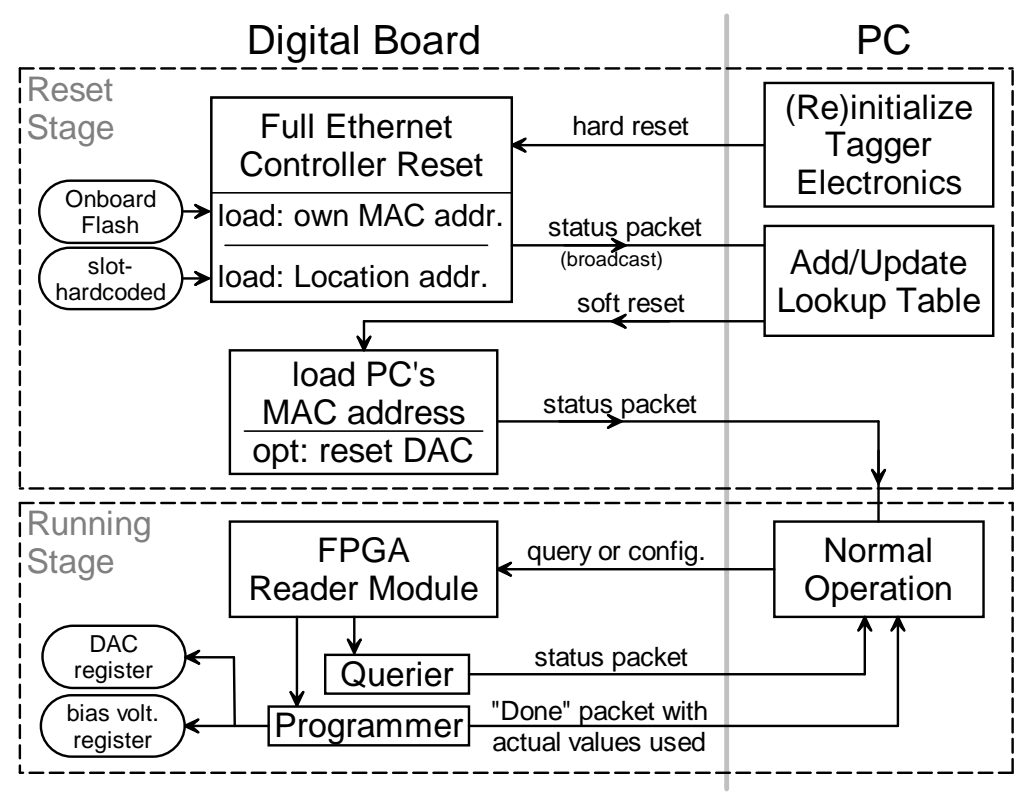

Fig. 3.12: This data flow diagram shows the procedure for initializing the detector electronics and the transition to the normal operating regime. Note how this process does not presuppose any records of the MAC or geographic addressed of electronics bundles. The control board reports its MAC address and its geographic address (encoded by its physical slot) allowing the computer to build a lookup table. The system then switches to unicast for further communication.

broadcast the standard "status" packet. The controlling PC records the MAC addresses of the received packets along with the geographic address the boards reported and build a table, cross-referencing the MAC address with the focal plane segment. This "roll call" can be repeated any time for the computer's sake without reseting the detector by issuing a broadcast "query" packet from the PC, which prompts another round of "status" packets. The startup sequence can be finished off with a "soft reset" instruction from the PC. Ordinarily, this packet is used to clear the control board's registers, including the DAC voltage values. Its other purpose it to acquaint the control boards with their master-PC. The control boards record the MAC address of the computer that issued the "soft reset" packet 
and henceforth communicate its reports directly to the PC (unicast).

Note that both the MAC address and our custom geographic address schemes include the notion of broadcast and unicast. Setting the recipient geographic address to $0 \mathrm{xFF}$ is defined as a broadcast packet. It simplified the control board's FPGA firmware to avoid setting the receiver filter hash table. So, by default the control boards will receive and examine any packet picked up on the network, but the packet is thrown away if the geographic address in the payload does not match its own and does not indicate a broadcast. This limitation should be a cause for some caution about the networking environment set up for this detector. Without a filter, the small receive buffer may not be able to handle the traffic of a normal network. Aside from this, discrimination based on the one byte of the geographic address may not be a sufficiently safe filter to avoid accidental manipulation of the detector by extraneous network traffic. Modern protocols used on the network can be examined to see if the possible geographic addresses and the packet types of our protocol (i.e. first two bytes of the payload) coincide with any of their codes.

During normal operation, the $\mathrm{PC}$ can issue query or program packets at any time, either broadcast to all or directed to a particular board. The structure of the packets in this custom communication protocol is shown in Figure 3.13.

The figure also illustrates the programming packet, in which the PC issues a set of 32 codes for the DAC, 30 of which set the bias voltages on the SiPMs, one is reserved as a read-back line ("DAC health") and the last sets the amplifier board gain mode. The DAC channels can be changed selectively by using a 4byte mask coded into the packet. Only the channels flagged with a ' 1 ' bit in the mask are set to the value specified in the packet. The program packet is always answered with a "data" packet, reporting back the DAC voltage codes used to 

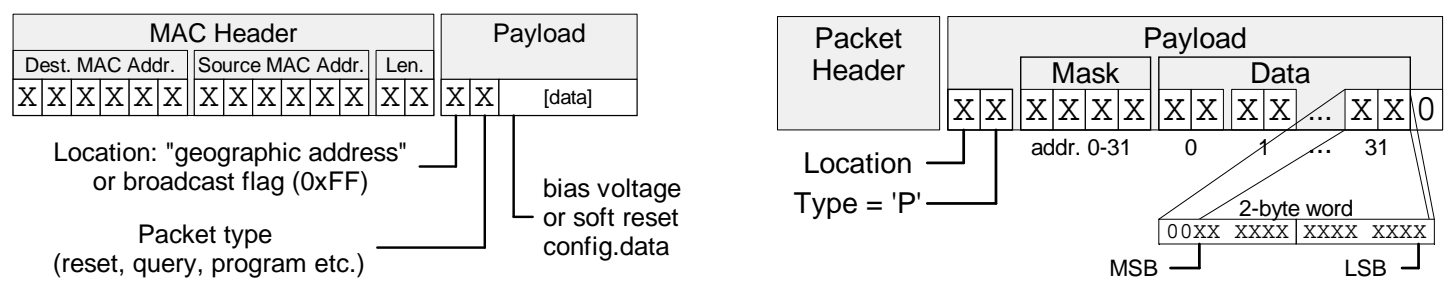

Fig. 3.13: Format of Ethernet packets sent to the detector. Left: General format of Ethernet packets used to communicate with the Tagger Microscope. Right: Layout of the "program packet" data for SiPM bias voltage specification. Note that the channel numbering here represents DAC's channel numbers which map to actual SiPM channels depending on the trace-routing convenience on the electronics boards.

program the DAC. This is essentially a confirmation of the PC's instructions and not a voltage reading of the DAC's set values. (The DAC does not have such a read-back capability, hence a health line is reserved to check the code-to-voltage setting consistency.) The control board can overrule a PC's voltage setting if it exceeds the safe maximum limit set in the FPGA firmware. In this case, this maximum is given to the DAC and reported back to the PC. Effectively, the mask feature can be used to query the control board's presumed DAC values by sending a program packet with arbitrary voltage settings and zeroing the entire mask. What is received is then the DAC values from the FPGA register, representing the last historical setting to each channel. This is useful if the PC loses its own record of the current voltage settings in the detector and requires an inventory.

\subsection{Beam Test}

A beam test of the tagger microscope prototype was conducted in Hall B of Jefferson Lab downstream of its tagger magnet. The prototype included a single set of electronics and one fiber bundle with 5 columns. The amplifier board was instrumented with 19 Photonique SSPM-06x SiPMs, constituting readout 
for close to 4 fiber columns. Of these, one column had individual readout, but unfortunately had a disabled summed output plug. The sensors were housed in a light-tight enclosure complete with motor-actuated alignment stage for sweeping through the beam within a range of $1 \mathrm{~cm}$ and for angular alignment transverse to the dispersed electron beam. The beam test included the prototype digitizing and trigger electronics to be used in Hall D, allowing a very realistic test of the detector performance.

Readout of the prototype was triggered by a pair of fast PMTs reading out a scintillator mounted upstream of the scintillating fibers. Unfortunately this trigger paddle could not handle rates above $300 \mathrm{kHz}$. Additionally the trajectory of electrons at the location of the prototype was poorly understood, resulting in likely a small overlap between the trigger paddle and scintillator bundle, a factor of five lower rate of signals in fibers than expected from the facility's beamline simulation and poor alignment with respect to the fiber axis. These issues resulted, on average, in lower and variable amplitude pulses in the collected data. The problems were compensated with more statistics and selection of pulses based on amplitude range. The time resolution was then studied at different threshold levels with respect to the readout pulse height. (The leading edge discriminator threshold remained constant during the beam test; here the relative threshold level serves as a proxy for signal amplitude.) Among the recorded signals was the "accelerator RF" clock. Time resolution is then defined as the r.m.s. deviation between the accelerator bunch time reference and the recorded pulse in the prototype's scintillating fiber.

Figure 3.14 shows a distribution of time differences between accelerator RF and leading-edge discriminator-recorded timing of pulses in one prototype sum 
channel. The figure compares the resolution at two effective threshold levels. This is accomplished by selecting timing data from different amplitude height groups. Figure 3.15 then plots the derived time resolution as a function of discriminator threshold, defined as a fraction of the pulse height. It must be emphasized that low threshold samples in this analysis correspond to higher amplitude pulses due to a larger number of activated SiPM pixels. Thus, the dependence of time resolution on threshold as shown in Figure 3.15 is likely not dominantly a threshold effect, but a function of detected photon statistics.

Though the beam test did not measure the required time resolution of $200 \mathrm{ps,}$ extrapolation from these data suggests that this goal is achievable with the intended threshold level of around 30\%. Moreover, the right plot in Figure 3.14 shows that time resolution of about $360 \mathrm{ps}(\sim 75 \%$ threshold) already results in very small overlap in the distribution of timing measurements for electrons of adjacent beam bunches. This means that the desired accuracy of beam bunch identification by the tagger microscope will be achieved even with time resolution worse than the specified $200 \mathrm{ps}^{9}$

\footnotetext{
9 The conservative specification of 200 ps was made to ensure that the non-Gaussian tails of the pulse time distribution did not add significantly to the overlap.
} 

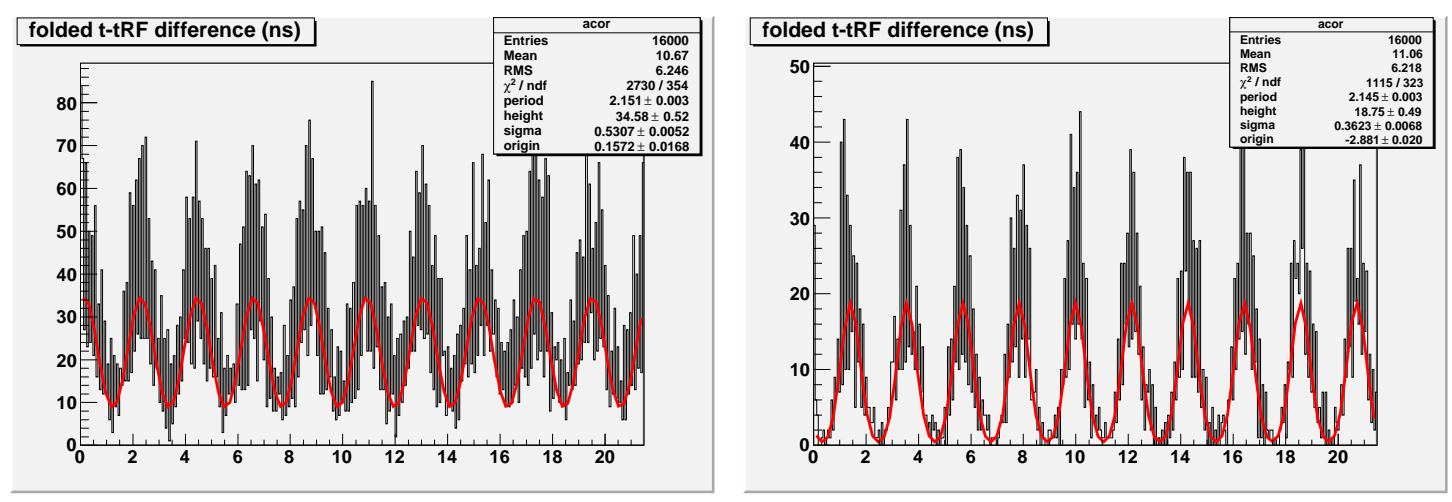

Fig. 3.14: Difference between the "accelerator RF" clock and the timing from pulse analysis is shown for a higher (left) and lower (right) effective thresholds with respect to the signal amplitude. The time resolution is clearly worse for the higher threshold. Overlap between pulse timing distributions indicates the rate of accelerator bunch misidentification.

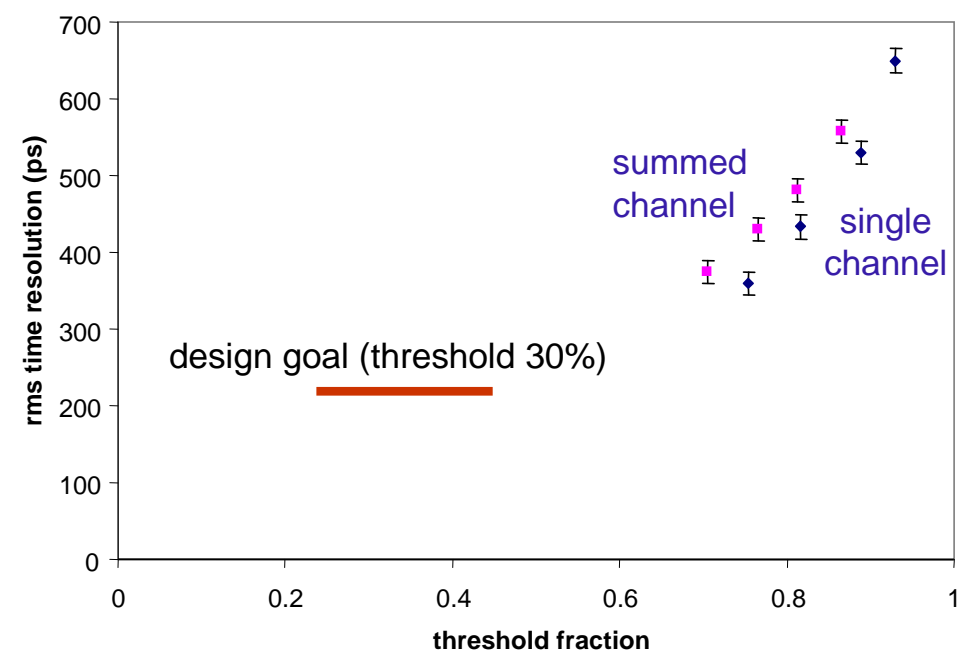

Fig. 3.15: Measured time resolution is shown as a function of the effective threshold used in analysis. Extrapolation to the designed threshold around $30 \%$ suggests that the 200 ps requirement can be met. 


\section{Chapter 4}

\section{Analysis}

\subsection{Introduction}

In the preparation of an experiment of this scale, it is important to develop the data analysis techniques ahead of data collection. Furthermore, ready analysis of incoming data is useful for guiding the experimental program in the short and long terms. Another reason to undertake an analysis of a simulated decay in a detector under construction is that it creates a useful feedback within the experimental group, helping to guide detector and software optimization. For these reasons, the study of one particular channel was undertaken: $\gamma p \rightarrow X p \rightarrow b_{1} \pi p$. In the flux tube model, this reaction is thought to be favored for decaying exotic states: $\pi_{1}, h_{2}, h_{2}^{\prime}, h_{0}, h_{0}^{\prime}$ [34]. Additionally, it is sensitive to beam polarization, so its simulation and analysis provides a natural check on the beam line parameters optimized in the development efforts described above.

A Monte Carlo (MC) study of this form that culminates in an Amplitude Analysis involves the following set of steps

1. generating events based on hypothetical or known physics

2. simulating the final state particles' interaction with the detector

3. reconstruction of the events based on the "hits" in the detector 
4. analysis of the various hypotheses suggested by the reconstruction in relation to the sought physics channel and suppression of background

5. amplitude fit to the remaining event set

Of course, this mirrors the actual measurement process, substituting only the first two steps with simulation. The final step, the actual Amplitude Analysis, actually takes in two event samples: a "physics" event set and an "acceptance" event set. The latter is generated with the same steps except that the original MC event is sampled from probability distributions that only include the phase space of the final state particles. No resonance structures or angular correlations based on definite states of angular momentum are present. Simulating and analyzing this set adds information about the detector acceptance - critical for separating correlations from detector sensitivity and actual physics processes.

The physical amplitudes in the fit of the last step are also the source of the physics simulated in the beginning. ${ }^{10}$ For this reason, the following discussion will begin with the construction of production and decay amplitudes. First, some general relations will be given for deriving partial wave amplitudes in the Helicity Formalism with the assumptions of the Isobar Model. Application of this to the $b_{1} \pi$ study will be outlined and then the implementation will be covered. The discussion will then turn to producing a realistic data set, mixed with other physics and background processes and then the steps of detector reconstruction. Techniques of parallel computing used to carry out this study will be discussed along with the final results.

\footnotetext{
${ }^{10}$ Of course, an experimentalist analyzing real data has no knowledge of the original basis set of amplitudes that generated the events. At this stage, however, confirming the consistency of the analysis framework requires simulation and fits with the same known basis of amplitudes. Future studies could involve exercises such as blinding the analyst to the basis and precise mixture of states.
} 


\subsection{Angular Amplitudes: General Relations}

\subsubsection{Angular Distribution of a Two-Body Decay}

Consider the two-body decay of a state with angular momentum quantum numbers (QN) $J, M$. Specifically, we want to know the amplitude of this state for having daughter 1 with momentum direction $\Omega=(\phi, \theta)$ in the center of mass reference frame, and helicity $\lambda_{1}$, while daughter 2 has direction $-\Omega=(\phi+\pi, \pi-\theta)$ and helicity $\lambda_{2}$.

Let $U$ be the decay operator from the initial state into the given 2-body final state. Intermediate between the at-rest initial state of quantum numbers $J, M$ and the final plane-wave state is a basis of outgoing waves describing the outgoing 2-body state in a basis of good $J, M$ and helicities. Insertion of the complete set of intermediate basis vectors, and summation over all intermediate $J, M$ gives

$$
\left\langle\Omega \lambda_{1} \lambda_{2}|U| J M\right\rangle=\left\langle\Omega \lambda_{1} \lambda_{2} \mid J M \lambda_{1} \lambda_{2}\right\rangle\left\langle J M \lambda_{1} \lambda_{2}|U| J M\right\rangle
$$

There is an alternate way to describe the final state. Instead of specifying the final-state particles' spin state via their helicities, we can first couple their spins together to obtain total spin $S$, which is independent of their momentum direction, and couple $S$ to their relative orbital angular momentum $L$ to obtain their total angular momentum $J$. The helicity basis and the $L, S$ basis are each

individually complete and orthonormal within themselves. Following the above 
expression, let us insert a sum over the $L, S$ basis.

$$
\begin{aligned}
& \left\langle\lambda_{1} \lambda_{2}|U| J M\right\rangle=\sum_{L, S}\left\langle\Omega \lambda_{1} \lambda_{2} \mid J M \lambda_{1} \lambda_{2}\right\rangle\left\langle J M \lambda_{1} \lambda_{2} \mid J M L S\right\rangle\langle J M L S|U| J M\rangle \\
= & \sum_{L, S}\left[\sqrt{\frac{2 J+1}{4 \pi}} D_{M \lambda}^{J *}(\Omega)\right]\left[\sqrt{\frac{2 L+1}{2 J+1}}\left(\begin{array}{cc|c}
L & S & J \\
0 & \lambda & \lambda
\end{array}\right)\left(\begin{array}{cc|c}
S_{1} & S_{2} & S \\
\lambda_{1} & -\lambda_{2} & \lambda
\end{array}\right)\right]\left[a_{L S}^{J}\right]
\end{aligned}
$$

where $\lambda=\lambda_{1}-\lambda_{2}, \Omega=(\phi, \theta, 0)$ and the double-stacked symbols are ClebshGordan (CG) coefficients. ${ }^{11}$ The expressions in square brackets correspond to the "bra-ket" terms above. The product of CG coefficients in the second set of brackets on the right-hand side represent the overlap between the basis vectors in the helicity and $L, S$ basis, which turns out to be independent of $M$, as required by rotational invariance. A full derivation is available in [11]. This expression holds regardless of what axis is used to define the quantization direction for $M$. However, this axis must correspond with the z-axis of the reference frame in which the plane wave direction $\Omega$ is defined. The right-most term represents the coupling coefficient for transition of a $J, M$ state to a state with the given $L, S$ quantum numbers. As discussed below, this coefficient is a function of the invariant mass.

$11\left(\begin{array}{cc|c}j_{1} & j_{2} & J \\ m_{1} & m_{2} & M\end{array}\right) \equiv\left\langle j_{1} j_{2} m_{1} m_{2} \mid j_{1} j_{2} J M\right\rangle$ 


\subsubsection{Isospin Projections}

One must also take into account the various ways that the isospin of the daughters can add up to the isospin quantum numbers of the parent, requiring a term:

$$
C^{a, b}=\left(\begin{array}{cc|c}
I^{a} & I^{b} & I \\
I_{z}^{a} & I_{z}^{b} & I_{z}^{a}+I_{z}^{b}
\end{array}\right)
$$

where $a=1$ and $b=2$ refer to the daughter index. If the two non-identical daughter particles belong to the same isospin multiplet, there is a constraint introduced between orbital angular momentum and total isospin that follows from the symmetry of exchanging the two particle identities, because $180^{\circ}$ rotation is equivalent to the exchange of the daughter identities $(a, b$ becoming $b, a)$. For example, for

a two-pion final state in an even- $L$ angular wave, only even $I$ is allowed, and for an odd- $L$ angular wave, only odd $I$ is allowed. Because of this, it is convenient to define a symmetrized variant of the $C$ coefficients defined above,

$$
C(L)=\frac{1}{\sqrt{2}}\left[C^{a, b}+(-1)^{L} C^{b, a}\right]
$$

It should be kept in mind that this $C(L)$ is only applicable for particle pairs in the same isospin multiplet.

\subsubsection{Reflectivity}

Beside rotational invariance, parity is also a good symmetry of strong hadron dynamics. In the case described above of the decay of a single particle at rest into two daughters, parity conservation places constraints between different final state amplitudes. Instead of considering the parity operator directly, it is convenient 
to consider the reflectivity operator $\mathbb{R}$. Reflectivity is the product of parity with a $180^{\circ}$ rotation about the y axis, which is defined to be normal to the production plane. The advantage of using this more complicated operator to express the constraints of parity is that a general two-particle plane wave basis can be constructed out of eigenstates of reflectivity, whereas a complete plane-wave basis of parity eigenstates is possible only in the restricted case that daughters 1 and 2 are identical. Regardless of the additional rotation, the basic constraint of reflectivity conservation is nothing more than parity conservation plus rotational invariance.

Acting on a state of good $J, M$, the reflectivity operator has a particularly simple effect.

$$
\mathbb{R}|J M\rangle=P(-1)^{J-M}|J-M\rangle
$$

where $P$ is the intrinsic parity of the system. The eigenstates of the reflectivity operator are formed out of states of good $J, M$ as follows:

$$
|J M \epsilon\rangle=|J M\rangle+\epsilon P(-1)^{J-M}|J-M\rangle
$$

where $\epsilon= \pm 1$ for a bosonic system and $\epsilon= \pm i$ for a fermionic system. It follows that

$$
\mathbb{R}|J M \epsilon\rangle=\epsilon(-1)^{2 J}|J M \epsilon\rangle
$$

\subsection{Application}

Let us proceed to the construction of an amplitude for a process involving photoproduction of a meson in recoil from an unpolarized proton and the subsequent decay into $b_{1} \pi \rightarrow \omega \pi \pi \rightarrow 5 \pi$ (including only the dominant decay modes.) Follow- 
ing the Isobar Model, decays are formulated as two-body processes with no final state interactions. The overall diagram for this process is shown in Figure 4.1. Amplitudes for other decay channels can be constructed analogously.

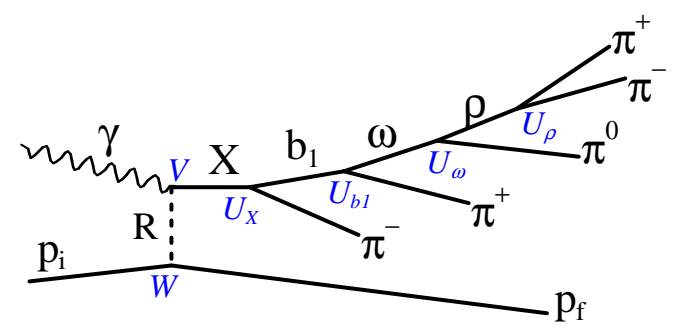

Fig. 4.1: Feynman diagram for photo-production of a resonance $X$ in recoil from a proton, with the decay of the resonance to $b_{1} \pi$. Only the dominant modes of $b_{1}$ and $\omega$ decays are included into the overall process amplitude. The inverse isospin combination to this diagram, $b_{1}^{-} \pi^{+}$, is included.

\subsubsection{Photon-Reggeon-Resonance vertex}

Consider the t-channel production of a resonance from the photon and reggeon in the reflectivity basis, consisting of plane-wave states constructed to be eigenstates of the reflectivity operator. This turns out, in the case for the photon, to correspond to the usual linear polarization basis $|x\rangle$ and $|y\rangle$. Let the $x(y)$ linear polarization states be denoted as $\epsilon=-1 \quad(\epsilon=+1)$.

$$
\begin{gathered}
\left|\epsilon_{\gamma}\right\rangle=\sqrt{\frac{-\epsilon_{\gamma}}{2}}\left(|1-1\rangle-\epsilon_{\gamma}|1+1\rangle\right) \\
\mathbb{R}\left|\epsilon_{\gamma}\right\rangle=\epsilon_{\gamma}\left|\epsilon_{\gamma}\right\rangle
\end{gathered}
$$

The strong interaction Hamiltonian respects reflectivity, so the production operator $V$ should commute with $\mathbb{R}$. 


$$
\begin{aligned}
V & =\mathbb{R}^{-1} V \mathbb{R} \\
\therefore\left\langle J M \epsilon|V| \epsilon_{\gamma} ; \lambda_{R} \epsilon_{R} ; \Omega_{0}\right\rangle & =\left\langle J M \epsilon\left|\mathbb{R}^{-1} V \mathbb{R}\right| \epsilon_{\gamma} ; \lambda_{R} \epsilon_{R} ; t, s ; \Omega_{0}\right\rangle \\
& =\epsilon \epsilon_{\gamma} \epsilon_{R}\left\langle J M \epsilon|V| \epsilon_{\gamma} ; \lambda_{R} \epsilon_{R} ; \Omega_{0}\right\rangle
\end{aligned}
$$

Acting with the reflectivity operator on initial and final state brings out the reflectivity eigenvalues of the resonance, photon and Reggeon. This result leads to a constraint $\epsilon=\epsilon_{\gamma} \epsilon_{R}$ that embodies parity conservation in this decay. It is convenient to adopt the Gottfried-Jackson frame. In particular, we boost into the reference frame of the produced resonance and orient the coordinate system such that the photon is in the $+\mathrm{z}$ direction and the $\mathrm{x}$-axis is co-planar to the recoiling proton, thus defining $\mathrm{xz}$ as the production plane. A consequence of this choice is that $m=\lambda_{\gamma}-\lambda_{R}$.

It is convenient to express the above matrix element as

$$
\left\langle J M \epsilon|V| \epsilon_{\gamma} ; \lambda_{R} \epsilon_{R} ; \Omega_{0}\right\rangle=v_{J, m, \epsilon ; \epsilon_{\gamma} ; \lambda_{R}, \epsilon_{R}}
$$

so that the indexed coefficient $v$ specifies the couplings together with the consequences of angular momentum and parity conservation. The function $v$ is implicitly dependent upon the kinematic variables $s$ and $t$. This dependence will be made explicit in a following section, after the matrix element for the baryon vertex has been studied.

To express the initial photon linear polarization state in the reflectivity basis, we relate the linear polarization bases in the laboratory and Gottfried-Jackson coordinate systems: 


$$
\begin{aligned}
\left(\begin{array}{c}
\epsilon_{\gamma}=-1 \\
\epsilon_{\gamma}=+1
\end{array}\right) & =\left(\begin{array}{cc}
\cos \alpha & -\sin \alpha \\
\sin \alpha & \cos \alpha
\end{array}\right)\left(\begin{array}{c}
x_{\mathrm{lab}} \\
y_{\mathrm{lab}}
\end{array}\right) \\
& =\frac{1}{\sqrt{2}}\left(\begin{array}{cc}
e^{-i \alpha} & e^{i \alpha} \\
i e^{-i \alpha} & -i e^{i \alpha}
\end{array}\right)\left(\begin{array}{l}
|1-1\rangle \\
|1+1\rangle
\end{array}\right)_{\mathrm{lab}}
\end{aligned}
$$

where $\alpha=\phi$, the azimuthal angle of the production plane in the lab system.

\subsubsection{Decay of t-channel resonance $X$}

We can apply the above recoupling relations to write down the amplitude at each vertex of the decay tree. These amplitudes are defined within a series of coordinate systems, each defined with respect to its ancestor in the decay chain. The chain starts with resonance X decaying in the Gottfried-Jackson frame, in the direction $\Omega_{b 1}$. To find the decay frame of the $b_{1}$, we perform the rotation $\left(\phi_{b 1}, \theta_{b 1}, 0\right)$ (Euler convention $\left.\mathrm{z}, \mathrm{y}^{\prime}, \mathrm{z}^{\prime \prime}\right)$ then boost into the rest frame of the $b_{1}$. To find the decay frame of the $\omega$, we rotate by $\left(\phi_{\omega}, \theta_{\omega}, 0\right)$, then boost into the $\omega$ rest frame. The three-body decay of the $\omega$ can be treated without loss of generality as a decay into a charged di-pion and a neutral pion, provided that a complete sum over states of the free di-pion system is performed. For notational simplicity, the dipion is represented below by the symbol $\rho$, which should not be confused with the physical $\rho(770)$ resonance. The cascade of decay frames continues through the $\omega$ decay by definition of the decay angles $\left(\phi_{\rho}, \theta_{\rho}, 0\right)$, and finally $\left(\phi_{\pi}, \theta_{\pi}, 0\right)$. The selection rules for $\omega$ decay require that the charged di-pion system be in an overall isovector state, which excludes even orbital angular momentum between the $\pi^{+}$ and $\pi^{-}$. This requires that the di-pion system be antisymmetric in decay angles, 
so it turns out not to matter whether one uses the $\pi^{+}$or $\pi^{-}$member of the pair to define the angles $\left(\phi_{\pi}, \theta_{\pi}, 0\right)$.

This procedure results in the decay particle's quantization axis being the same as the momentum direction in the parent's frame, thus forcing the "M" quantum number in the decay frame to be equal to its helicity $\lambda$ used in the parent's frame. Substitutions of known quantum numbers are made as necessary below: pions in the final state are given zero helicities and spin of $b_{1}$ and $\omega$ are put in from the start.

$\left\langle\Omega_{b_{1}} \lambda_{b_{1}} 0\left|U_{X}\right| J_{X} M_{X}\right\rangle=\sum_{L_{X}}\left[\sqrt{\frac{2 J_{X}+1}{4 \pi}} D_{M_{X} \lambda_{b_{1}}}^{J_{X *}^{*}}\left(\Omega_{b_{1}}\right)\right]\left[\sqrt{\frac{2 L_{X}+1}{2 J_{X}+1}}\left(\begin{array}{cc|c}L_{X} & 1 \\ 0 & \lambda_{b_{1}} \mid J_{b_{1}}\end{array}\right)\right] u_{L_{X} 1}^{X: J_{X}}$

$\left\langle\Omega_{\omega} \lambda_{\omega} 0\left|U_{b_{1}}\right| 1 M_{b_{1}=\lambda_{b_{1}}}\right\rangle=\sum_{L_{b_{1}}}\left[\sqrt{\frac{2 J_{b_{1}}+1}{4 \pi}} D_{M_{b_{1}} \lambda_{\omega}}^{1 *}\left(\Omega_{\omega}\right)\right]\left[\sqrt{\frac{2 L_{b_{1}}+1}{2 J_{b_{1}}+1}}\left(\begin{array}{cc|c}L_{b_{1}} & 1 & 1 \\ 0 & \lambda_{\omega} & \lambda_{\omega}\end{array}\right)\right] u_{L_{b_{1}} 1}^{b_{1}: 1}$

$\left\langle\Omega_{\rho} \lambda_{\rho} 0\left|U_{\omega}\right| 1 M_{\omega=\lambda_{\omega}}\right\rangle=\sum_{L_{\omega} J_{\rho}}\left[\sqrt{\frac{2 J_{\omega}+1}{4 \pi}} D_{M_{\omega} \lambda_{\rho}}^{1 *}\left(\Omega_{\rho}\right)\right]\left[\sqrt{\frac{2 L_{\omega}+1}{2 J_{\omega}+1}}\left(\begin{array}{cc|c}L_{\omega} & J_{\rho} \mid \\ 0 & \lambda_{\rho} \mid \lambda_{\rho}\end{array}\right)\right] u_{L_{\omega} J_{\rho}}^{\omega: 1}$ 


$$
\begin{aligned}
\left\langle\Omega_{\pi} 00\left|U_{\rho}\right| J_{\rho} M_{\rho}=\lambda_{\rho}\right\rangle & =\sum_{L_{\rho}}\left[\sqrt{\frac{2 J_{\rho}+1}{4 \pi}} D_{M_{\rho} 0}^{J_{\rho} *}\left(\Omega_{\pi}\right)\right]\left[\sqrt{\frac{2 L_{\rho}+1}{2 J_{\rho}+1}}\left(\begin{array}{cc|c}
L_{\rho} & 0 & J_{\rho} \\
0 & 0 & 0
\end{array}\right)\right] u_{L_{\rho} 0}^{\rho: J_{\rho}} \\
& =Y_{M_{\rho}}^{J_{\rho}}\left(\Omega_{\pi}\right) u_{J_{\rho} 0}^{\rho: J_{\rho}}
\end{aligned}
$$

A further simplification is possible in the $\omega$ and $\rho$ systems. The spin- 1 and negative parity of $\omega$ lock the $L_{\omega}$ and $J_{\rho}$ to be the same odd integer: $L_{\omega}=J_{\rho}=$ $1, L_{\omega}=J_{\rho}=3, \ldots$ Experimental evidence so far suggests that only the P-wave $\left(L_{\omega}=1\right)$ contributes, so values other than 1 may be neglected.

\subsubsection{Assembly of the full amplitude}

Putting together the amplitudes discussed above, we arrive at the complete angulardependent amplitude of photo-production of resonance $\mathrm{X}$ and decay to the given final state. The final expression for the measured cross section becomes:

$\frac{d^{8} \sigma}{d \Omega_{b 1} d \Omega_{\omega} d \Omega_{\rho} d \Omega_{\pi}}=\frac{1}{16 \pi^{2} s}\left|T_{f i}\right|^{2}\left(\frac{p_{f}}{p_{i}}\right)\left(\frac{q_{b 1} d m_{X}}{16 \pi^{3}}\right)\left(\frac{q_{\omega} d m_{b 1}}{16 \pi^{3}}\right)\left(\frac{q_{\rho} d m_{\omega}}{16 \pi^{3}}\right)\left(\frac{q_{\pi} d m_{\rho}}{16 \pi^{3}}\right)$

where $p_{i}\left[p_{f}\right]$ is the target [recoil] nucleon momentum in the center of mass frame of the overall reaction. In terms of the individual decay matrix elements introduced earlier, the $\mathrm{T}$ matrix element can be written as 


$$
\begin{gathered}
T_{(f)(i)}=T_{\left(\mathbf{q}_{\pi} \mathbf{q}_{\rho} \mathbf{q}_{\omega} \mathbf{q}_{b_{1}} \mathbf{p}_{f} \epsilon_{f}\right)\left(\mathbf{k}_{\gamma} \epsilon_{\gamma} \mathbf{p}_{i} \epsilon_{i}\right)} \\
=\sum_{R, \lambda_{R}, \epsilon_{R} ; \lambda_{b_{1}}, \lambda_{\omega}, \lambda_{\rho}}\left\langle\mathbf{q}_{\pi} 00 ; \mathbf{q}_{\rho} \lambda_{\rho} 0 ; \mathbf{q}_{\omega} \lambda_{\omega} 0 ; \mathbf{q}_{b_{1}} \lambda_{b_{1}} 0|U V| \epsilon_{\gamma} ; \lambda_{R} \epsilon_{R} ; \Omega_{0}\right\rangle \times \\
\left\langle\lambda_{R} \epsilon_{R} ; \Omega_{0} ; \mathbf{p}_{\mathbf{f}}, \epsilon_{f}|W| \mathbf{p}_{\mathbf{i}}, \epsilon_{i}\right\rangle
\end{gathered}
$$

To obtain the second line in the above equation, we factorized the T operator into two vertex factors $U V$ and $W$, and inserted between them a sum over a complete set of intermediate exchanges $R$ represented as plane waves moving along the $-z$ axis. The upper vertex operator has been written as $U V$ in anticipation of its further factorization into the primary resonance production operator $V$ and its decay operator $U$. Polarizations of all particles are represented by their respective reflectivity quantum numbers $\epsilon$. For the nucleon, the reflectivity is a complete description of its spin state. For reactions involving higher spin baryons, it would need to be supplemented by an additional $|\lambda|$ quantum number.

$$
\begin{aligned}
T_{(f)(i)}= & \sum_{R, \lambda_{R}, \epsilon_{R}, \lambda_{b_{1}}, \lambda_{\omega}, \lambda_{\rho}} \\
& \left\langle\mathbf{q}_{b 1} \lambda_{b_{1}} 0\left|U_{X}\right| J_{X} M_{X} \epsilon_{X}\right\rangle\left\langle\mathbf{q}_{\omega} \lambda_{\omega} 0\left|U_{b_{1}}\right| 1, \lambda_{b_{1}}\right\rangle \times \\
X, M_{X}, \epsilon_{X} ; \epsilon_{i}, \epsilon_{f} & \\
& \left\langle\mathbf{q}_{\rho} \lambda_{\rho} 0\left|U_{\omega}\right| 1, \lambda_{\omega}\right\rangle\left\langle\mathbf{q}_{\pi} 00\left|U_{\rho}\right| J_{\rho}, \lambda_{\rho}\right\rangle \times \\
& \left\langle J_{X} M_{X} \epsilon_{X}|V| \epsilon_{\gamma} ; \lambda_{R} \epsilon_{R} ; \Omega_{0}\right\rangle \times \\
& \left\langle\lambda_{R} \epsilon_{R} ; \Omega_{0} ; \mathbf{p}_{\mathbf{f}}, \epsilon_{f}|W| \mathbf{p}_{\mathbf{i}}, \epsilon_{i}\right\rangle
\end{aligned}
$$

Parity conservation requires that $\epsilon_{X}=\epsilon_{\gamma} \epsilon_{R}$ and $\epsilon_{R}=\epsilon_{i} \epsilon_{f}$. The last two 
matrix elements in the expression above for $T_{f i}$ are not known a priori, so we parameterized them into a pair of unknown functions $v(s, t)$ and $w(s, t)$.

$$
\begin{aligned}
& v_{\lambda_{R}, \epsilon_{R}}^{X, M_{X}, \epsilon_{X}}(s, t)=\left\langle J_{X} M_{X} \epsilon_{X}|V| \epsilon_{\gamma} ; \lambda_{R} \epsilon_{R} ; \Omega_{0}\right\rangle \\
& w_{\lambda_{R} \epsilon_{R} ; \epsilon_{i}}(s, t)=\left\langle\lambda_{R} \epsilon_{R} ; \Omega_{0} ; \mathbf{p}_{\mathbf{f}}, \epsilon_{f}|W| \mathbf{p}_{\mathbf{i}}, \epsilon_{i}\right\rangle
\end{aligned}
$$

\subsubsection{Proton states and individual decay amplitudes}

An average over the target proton initial state will be necessary to compute the cross section. Also, because the polarization of the recoiling proton cannot be measured, a sum over the proton final states must be done. This can be represented as

$$
\frac{d^{8} \sigma}{d \Omega_{b 1} d \Omega_{\omega} d \Omega_{\rho} d \Omega_{\pi}} \propto \sum_{\epsilon_{\gamma} \epsilon_{\gamma}^{\prime} \epsilon_{i} \epsilon_{f} \epsilon_{i}^{\prime} \epsilon_{f}^{\prime}} \rho_{\epsilon_{\gamma} \epsilon_{\gamma}^{\prime}} \rho_{\epsilon_{i} \epsilon_{i}^{\prime}} \delta_{\epsilon_{f} \epsilon_{f}^{\prime}} T \underset{\mathbf{q}_{\pi} \mathbf{q}_{\rho} \mathbf{q}_{\omega} \mathbf{q}_{b 1} \mathbf{p}_{f} \epsilon_{f}, T_{\mathbf{k}_{\gamma} \epsilon_{\gamma} \mathbf{p}_{i} \epsilon_{i}}^{*}}{\mathbf{q}_{\pi} \mathbf{q}_{\rho} \mathbf{q}_{\omega} \mathbf{q}_{b 1} \mathbf{p}_{f} \epsilon_{f}^{\prime}}
$$

where density matrices $\rho$ represent the initial state particles' spin states. The unpolarized target presents an initial state with both reflectivities equally likely, resulting in $\rho_{\lambda_{i} \lambda_{i}^{\prime}}=\frac{1}{2} \delta_{\lambda_{i} \lambda_{i}^{\prime}}$.

In analogy to the reflectivity conservation relation shown above for the $V$ vertex, there is a similar relation for the $W$ vertex: $\epsilon_{R}=\epsilon_{i} \epsilon_{f}$ Identification of $\epsilon_{i}$ with $\epsilon_{i}^{\prime}$ and $\epsilon_{f}$ with $\epsilon_{f}^{\prime}$ implies that only terms with $\epsilon_{R}=\epsilon_{R}^{\prime}$ survive in the sum over exchange quantum numbers. The quadratic sum expression above for the differential cross section invokes a double-sum over all of the internal quantum numbers that have been introduced, plus the spins of the initial and final nucleons. 
This sum is of the generic form

$$
\sum_{X, M_{X}, \cdots} \cdots \sum_{\epsilon_{i} \epsilon_{f}} \sum_{X, M_{X}, \cdots} \cdots \sum_{\epsilon_{i} \epsilon_{f}}\left|w_{\lambda_{R} \epsilon_{R} ; \epsilon_{i} \epsilon_{f}}\right|^{2}
$$

Note that the measured cross section only depends on the summed modulus squared of the $w$ coefficients, independent of the couplings to the individual nucleon helicity states. Because of this, the sum over nucleon helicities can be dropped, and the $w$ factors absorbed into the $v$ coefficients. Thus the final expression for the differential cross section contains no reference to the quantum numbers $\epsilon_{i}, \epsilon_{f}$, nor to any $w$ coefficients. It is important to recognize that this simplification of the cross section is only possible in the case of an unpolarized target and no recoil polarimetry.

\subsubsection{Mass dependence}

Expressions for the angular dependence of the matrix elements of $U_{X}, U_{b 1}, U_{\omega}$, and $U_{\rho}$ have already been written down above, in terms of the unknown massdependent factors: $u^{\rho}, u^{\omega}, u^{b_{1}}, u^{X}$ The mass dependence of the $u^{X}$ factor can be written in terms of a standard relativistic Breit-Wigner resonance lineshape as follows, although often its mass dependence is determined empirically by binning in the mass of $X$, and fitting each bin independently. In a global mass-dependent fit, the central mass and width of $X$ are free parameters in the fit. The remaining factors are assigned standard resonance forms, with their central mass, width and partial wave matrix elements fixed to agree with the established values for these resonances. Note that compared to the notation above, repeated and constant indices have been dropped in the expressions below. 


$$
\begin{gathered}
u_{L_{X}}^{X: J_{X}}\left(m_{X}\right)=u_{L_{X}}^{X: J} B W_{L_{X}}\left(m_{X} ; m_{X}^{0}, \Gamma_{X}^{0}\right) \\
u_{L_{b_{1}}}^{b_{1}}\left(m_{b 1}\right)=u_{L_{b_{1}}}^{b_{1}} B W_{L_{b_{1}}}\left(m_{b 1} ; m_{b 1}^{0}, \Gamma_{b 1}^{0}\right) \\
u_{L_{\omega}}^{\omega}\left(m_{\omega}\right)=u_{L_{\omega}}^{\omega} B W_{L_{\omega}}\left(m_{\omega} ; m_{\omega}^{0}, \Gamma_{\omega}^{0}\right) \\
u_{J_{\rho}}^{\rho}\left(m_{\omega}\right)=u_{J_{\rho}}^{\rho} B W_{J_{\rho}}\left(m_{\rho} ; m_{\rho}^{0}, \Gamma_{\rho}^{0}\right)
\end{gathered}
$$

The mass-dependent terms in the expressions above are given by the explicitly unitary Breit-Wigner form:

$$
B W_{L}\left(m ; m_{0}, \Gamma_{0}\right)=\frac{m_{0} \Gamma_{L}(m)}{m_{0}^{2}-m^{2}-i m_{0} \Gamma_{L}(m)}
$$

where,

$$
\Gamma_{L}\left(m ; m_{0}, \Gamma_{0}\right)=\Gamma_{0} \frac{m_{0}}{m} \frac{q}{q_{0}} \frac{F_{L}^{2}(q)}{F_{L}^{2}\left(q_{0}\right)}
$$

where $q$ is the breakup momentum of the daughter particles in the rest frame of the parent particle, and $q_{0}$ is the same, evaluated at $m_{0}$.

$$
q(m)=\sqrt{\left(\frac{m^{2}+m_{1}^{2}-m_{2}^{2}}{2 m}\right)^{2}-m_{1}^{2}}
$$

The functions $F_{L}(q)$ are the angular momentum barrier factors that are given in the literature [35]. The first few are listed below with $z=[q /(197 \mathrm{MeV} / \mathrm{c})]^{2}$ 


$$
\begin{aligned}
& F_{0}(q)=1 \\
& F_{1}(q)=\sqrt{\frac{2 z}{z+1}} \\
& F_{2}(q)=\sqrt{\frac{13 z^{2}}{(z-3)^{2}+9 z}} \\
& F_{3}(q)=\sqrt{\frac{277 z^{3}}{z(z-15)^{2}+9(2 z-5)^{2}}}
\end{aligned}
$$

\subsubsection{Describing $s$ and $t$ dependence}

It might be useful at some point to do a global fit to the data from all s,t bins. In such a case, it is useful to recall the expected behavior in high-energy peripheral production given by Regge theory.

$$
\sigma \sim s^{\alpha_{R}-1} e^{b_{R} t}
$$

where $\alpha_{R}$ is the intercept of the Regge trajectory for exchange particle $R$, and $b_{R}$ is the forward t-slope parameter for exchange trajectory $R$ at this value of $s$. Appending these factors to the above expression for the differential cross section, inside the sum over exchanges $R$, would allow data from all bins in $s$ and $t$ to be fitted in a single global fit.

\subsubsection{Summing over photon polarization}

The $\mathrm{T}$ Matrix, written in the photon reflectivity basis can be expanded in the

photon's lab frame helicity basis. Temporarily omitting indices not pertaining to the photon:

$$
T_{\epsilon_{\gamma}}=\sqrt{\frac{-\epsilon_{\gamma}}{2}}\left(T_{-1} e^{-i \alpha}-\epsilon_{\gamma} T_{+1} e^{i \alpha}\right)
$$


where,

$$
T_{ \pm 1}=\sum_{R, \lambda_{R} ; \lambda_{b_{1}}, \lambda_{\omega}, \lambda_{\rho}}\left\langle\mathbf{q}_{\pi} 00 ; \mathbf{q}_{\rho} \lambda_{\rho} 0 ; \mathbf{q}_{\omega} \lambda_{\omega} 0 ; \mathbf{q}_{b 1} \lambda_{b 1} 0|U|(1 \pm 1)_{\mathrm{lab}} ; \lambda_{R} \epsilon_{R} ; s, t\right\rangle
$$

Now, the average over the initial photon polarization states results in a cross section evaluated as follows:

$$
\begin{aligned}
\frac{d^{8} \sigma}{d \Omega_{b 1} d \Omega_{\omega} d \Omega_{\rho} d \Omega_{\pi}} & \propto \frac{1+g}{2}\left|\frac{1}{\sqrt{2}}\left(T_{-1} e^{-i \alpha}+T_{+1} e^{i \alpha}\right)\right|^{2} \\
& +\frac{1-g}{2}\left|\frac{i}{\sqrt{2}}\left(T_{-1} e^{-i \alpha}-T_{+1} e^{i \alpha}\right)\right|^{2} \\
& =\frac{1}{2}\left[\left|T_{-1}\right|^{2}+\left|T_{+1}\right|^{2}+g\left(T_{+1} T_{-1}^{*} e^{2 i \alpha}+T_{+1}^{*} T_{-1} e^{-2 i \alpha}\right)\right] \\
& =\frac{\left|T_{-1}\right|^{2}+\left|T_{+1}\right|^{2}}{2}+g \operatorname{Re}\left(T_{+1} T_{-1}^{*} e^{2 i \alpha}\right)
\end{aligned}
$$

where $g$ is the polarization fraction ranging from $1(100 \% \mathrm{x}$-polarized $)$ to 0 (unpolarized.)

\subsection{Implementation}

The amplitude constructed above has been coded for use with the Indiana University AmpTools package for calculations in Monte Carlo (MC) event generators and Amplitude Analysis fits [1]. The AmpTools package is a general, parallel computing-optimized toolkit written in $\mathrm{C}++$ for unbinned maximum likelihood fits with user-written amplitudes. These amplitudes are submitted for fits or event generation by means of classes that inherit from the package's "Amplitude" class. Organization of amplitudes into coherent and incoherent sum groups along with 
other parameters of MC generation and fits are specified in AmpTools-specific configuration files. Calculation of intensities and weighing-in of detector acceptance is performed by the toolkit.

This framework allows an amplitude to be constructed out of modular components. For example, an amplitude for the angular dependence may be coded separately from a Breit-Wigner amplitude for mass dependence. Multiplication of these "peer" amplitudes can then be specified in the configuration files. The work described here on the $b_{1} \pi$ channel makes some use of this. When used for MC event generation, a Breit-Wigner is invoked separately to shape the invariant mass distribution for the original meson $X$. (It is then omitted for mass-independent fits.) However, to allow the resonance shapes of child states to be composed of several orbital angular momentum components (e.g. $b_{1} \mathrm{~S}$ and D waves) a Breit-Wigner amplitude factor function was included explicitly inside the $b_{1} \pi$ amplitude code. Also, it is convenient to compute the polarization state coefficients $\left(\frac{1}{2}(1 \pm g)\right)$ separately, since this would be the only polarization-fraction dependent term in the amplitude. Separating out this piece would speed up any fit with the parameter left floating, since the rest of the expression would not need to be recomputed.

\subsubsection{Amplitude Parameters}

The internal amplitude coupling parameters $u^{\rho}, u^{\omega}, u^{b_{1}}$ are specified according to the current state of knowledge about these states.

- $\mathbf{u}^{\omega}$ : Lacking evidence for $\omega$ F-wave decay, we set the single coefficient $u_{L_{\omega}=1}^{\omega}=1.0$

- $\mathbf{u}^{\rho}: J_{\rho}\left(=L_{\rho}\right)$ restricted to odd integers, is further locked by the triangle inequality 
$\left(J_{\rho}+L_{\omega}<J_{\omega}=1\right)$ to equal $L_{\omega}$. Thus, we set the single coefficient: $u_{J_{\rho}=1}^{\rho}=$ 1.0

- $\mathbf{u}^{\mathbf{b}_{1}}: b_{1}$ decay to $\omega \pi$ is known to have the $\mathrm{D}$ to $\mathrm{S}$-wave ratio of $r=0.277 \pm$ 0.027 [32]. Thus:

$$
\begin{aligned}
& \circ u_{L_{b_{1}}=0}^{b_{1}}=\left(1+r^{2}\right)^{-1 / 2}=0.964 \\
& \circ u_{L_{b_{1}}=2}^{b_{1}}=r\left(1+r^{2}\right)^{-1 / 2}=0.267
\end{aligned}
$$

Though these defaults are built into the amplitude code, it is possible to pass these parameters in at run time and open them to variation during a fit. This is a general capability of fitting within the AmpTools framework, which allows a variable to be cast as an "AmpParameter" and "registered". Fixing or constraining such parameters is possible in a fit configuration file. Thus, it may be possible in the future to do general fits in which the amplitudes of the intermediate states are analyzed simultaneously with the overall fit to the $b_{1} \pi$ amplitude. In principle, analyses on several channels may be run simultaneously, allowing a global fit of the intermediate states. More immediately, run-time adjustment of these parameters helps check the amplitude code for basic orthogonality properties. Such procedures are discussed in the following section.

\subsubsection{Monte Carlo check of amplitude orthogonality}

To look for errors in the construction and coding of the amplitudes, a check for basic properties like orthogonality is useful. For this, inner products of amplitudes with different quantum numbers are necessary. But, designed to calculate intensities, the AmpTools framework is not currently set up to take these inner products. There is a way around this, however. Consider the intensity from the 
coherent sum of two amplitudes:

$$
I\left(c_{1}, c_{2}\right)=\left|c_{1} \Psi_{1}+c_{2} \Psi_{2}\right|^{2}=\left|c_{1}\right|^{2}\left|\Psi_{1}\right|^{2}+\left|c_{2}\right|^{2}\left|\Psi_{2}\right|^{2}+2 \operatorname{Re}\left(c_{1}^{*} c_{2} \Psi_{1}^{*} \Psi_{2}\right)
$$

The right-most term contains the sought expression. Thus, we can get the real part of that inner product by evaluating: $I(1,1)-I(1,0)-I(0,1)$. Setting one of the coefficients in the coherent sum to $i$ instead of $1(I(i, 1)-I(1,0)-I(0,1))$ yields the imaginary part.

There are two classes of quantum numbers to consider. The first corresponds to the orbital angular momentum of intermediate states (Ls) summed over during amplitude calculation. These states are ordinarily weighted with coefficients taken from previous data, but the coefficients can be set to the permutations of $1(i)$ and zero as above. The intensities can be recorded either by the amplitude calculator class or in the generator wrapper. The latter is cleaner but care must be taken to anticipate the amplitudes that vanish entirely. In this case an endless loop ensues with no events passing the accept/reject procedure and therefore no intensities recorded in the generator.

The second class of quantum numbers are "external" in that they are summed over by the AmpTools framework with terms set and initialized deliberately in the configuration file. One can set up amplitudes with the coefficient permutations listed above to be squared separately by specifying that they sum incoherently, intercepting their individual intensities and taking the difference as shown above.

Table 4.1 shows the results of the inner products taken with these methods on the $b_{1} \pi$ states. Three inner product results are shown for each quantum number subspace: two diagonal terms and the real part of the off-diagonal. (The imaginary parts were spot-checked and were seen to vanish with precision analogous to the 


\begin{tabular}{ccccc} 
q. n. & set of choices & $\Psi_{n} \cdot \Psi_{n}$ & $\Psi_{n^{\prime}} \cdot \Psi_{n^{\prime}}$ & $\Psi_{n} \cdot \Psi_{n^{\prime}}$ \\
\hline \hline$J_{\rho}$ & 1,3 & $0.409 \pm 0.004$ & $0.041 \pm 0.002$ & $(-1.6 \pm 2.4) \times 10^{-3}$ \\
$J_{\omega}$ & 1,3 & $0.409 \pm 0.004$ & $0.041 \pm 0.002$ & $(-1.6 \pm 2.4) \times 10^{-3}$ \\
$J_{b_{1}}$ & 0,2 & $0.220 \pm 0.002$ & $0.234 \pm 0.003$ & $(-2.6 \pm 3.7) \times 10^{-3}$ \\
\hline$J_{X}$ & 0,1 & $1.026 \pm 0.021$ & $0.662 \pm 0.035$ & $(2.6 \pm 0.9) \times 10^{-9}$ \\
$P_{X}$ & $+1,-1$ & $1.026 \pm 0.021$ & $1.740 \pm 0.035$ & $(-1.1 \pm 1.7) \times 10^{-9}$ \\
$\epsilon_{R}$ & $+1,-1$ & $1.734 \pm 0.035$ & $0.339 \pm 0.006$ & $(2.7 \pm 1.3) \times 10^{-9}$ \\
$\epsilon_{\gamma}$ & $+1,-1$ & $1.026 \pm 0.021$ & $1.734 \pm 0.035$ & $(2.1 \pm 1.6) \times 10^{-9}$ \\
\hline
\end{tabular}

Table 4.1: Diagonal and off-diagonal inner products of amplitudes. Note how the latter set ("external quantum numbers") have off-diagonal inner product on the order of $10^{-9}$. This likely indicates term by term cancellation in the integral. By comparison, the "internal quantum numbers" (those summed over) do not cancel term by term and approach zero as a function of the accumulated statistics.

real part. A thorough repetition of these calculations with higher statistics and closer attention to the imaginary component is planned.)

\subsection{Monte Carlo Event Generation}

\subsubsection{Event Generators}

We now turn to generating event sets for this study. A generator was written to produce event sets with five pions in the final state. This is essentially a wrapper around some other tools that do the principal calculations. This 5-pion generator employs a phase space generator for the final state particles that is based on the Raubold-Lynch method, implemented in a GlueX software class [24]. The events in this set may conform to the phase space distribution either through individual weights or by selecting equally-weighted samples appropriately. This is all that is necessary for the data set that introduces detector acceptance into the Amplitude Analysis. For generating physics signals, this is just the first step. Now, the intensity of each event is computed based on the amplitude set, which contributes 
another weight factor to each event. The final event set exported by the generator is shaped using an "accept/reject" procedure. The weight of each event becomes its probability of being accepted into the final set.

Amplitude calculations often take considerable computing resources. Rejecting many of the events in the final state makes for a very inefficient generator. Considerable gains in efficiency can be made with importance sampling. If the approximate shape of the distribution is known a priori, the method allows throwing events with a bias toward this approximate distribution while recording the biasing weight and factoring it into the "accept/reject" procedure. A limited use of importance sampling has been made in the early stages of this work to help simplify the code and facilitate error-checking. Specifically, importance sampling is used for the parent resonance shape, but not for the intermediate state lineshapes. However, the amplitude calculator has been optimized to bypass events in the far tails of the intermediate resonances, compensating somewhat for this limitation.

Since most of the useful physics events in the exotic meson spectroscopy program of GlueX would come from the $9 \mathrm{GeV}$ beam photon peak, the signal events were generated with this energy. (As a first step in this study, peak shape was not taken into consideration.) Competing physics events that contaminate the analysis are introduced with a Pythia-based GlueX tool called "bggen". The original Pythia library of the University of Lund models high-energy physics events in collisions relying on theory and models tuned to known phenomenology. This tool instruments the Pythia generator for the conditions of the GlueX experiment with a CB photon beam on a proton target. Although the input signal in this study is mono-energetic, a case can be made for including a broadly distributed background. The high rate on the tagger often produces more than one hit per 
detector event and it is left to the reconstruction algorithm to select the event rendition with the most plausible energy tag. Mistakes in this selection naturally allow events from other beam energies to contaminate the $9 \mathrm{GeV}$ signal sample. However, dealing with the challenge of multiple tagger hits is beyond the scope of this work. These first efforts in the analysis of a channel like $b_{1} \pi$ were made with the simplification that assumes that the energy of the production photon is precisely known. Thus, background was produced solely at $9 \mathrm{GeV}$.

Because simulation and reconstruction of events is computationally costly, a set of filters was developed to eliminate events with an insignificant likelihood of passing the analysis stage event selection. The criteria included charged track numbers too small to be interpreted as resulting from $b_{1} \pi$ decay and kinematics that result in intermediate $b_{1}$ and $\omega$ particle masses very far from their physical values. A study of false positives in background samples was performed to tune these filters to an optimum between having a most realistic background set and the computational and time costs.

\subsubsection{Generating Events: the Computing Challenge}

In spite of optimization in the amplitude calculator and the generators' event selection, current commodity computer hardware generates $b_{1} \pi$ with proper amplitudes at a rate of order $1 \mathrm{~Hz}$. Simulation and reconstruction programs process these events at about the same rate. Reconstruction efficiency of events with six final state particles in the current stage of GlueX reconstruction software is of order $1 \%$ (discussed in more detail in a later section). Now, consider that a good amplitude fit requires several thousand events and allow for about 20-60 resonance mass bins. The original background set to be simulated is of several orders higher 
cross-section. The acceptance (phase space) simulated event set also ought to be larger than the data set under fit. It is clear from these considerations that a massively parallel computing effort is necessary to carry out this study in a reasonable amount of time.

It has long been recognized that the efficient approach to parallel computing is through inexpensive commodity computer hardware, replicated to assemble computer arrays called beowulf clusters. This approach does not commit itself to increasing performance for running any one program, i.e. high performance computing (HPC). It rather focuses on high throughput computing (HTC), assuming that a complex problem can be divided into smaller parts and executed in parallel. Generally, under the HTC model, resources are managed and shared in a way that focuses on time-averaged computing power, hence the focus on overall throughput. Construction of HTC clusters with tens to hundreds of computers is common in university laboratories. Clusters of several thousand units are common to dedicated computing facilities housed in large companies, national labs and some dedicated academic computing centers. These clusters usually deploy batch computing software to distribute the segmented portions of a calculation job among the available computing units (usually defined as a slot for executing one single-threaded program - a CPU core.)

A cluster of about 100 computers (approximately 450 cores) with a total computing power of about 400 GFLOPS has been erected by the University of Connecticut Nuclear Physics group under Professor Richard Jones. This facility was built in collaboration with members from the Department of Statistics and includes resources funded by several U.S. National Science Foundation grants. However, such a facility is insufficient for these studies or the future data analysis. 
This facility and others that are being developed for GlueX do have the average computing capacity needed over the next several years for simulation and data analysis. These efforts, however, often include long idle periods when the scientists are engaged in other activities. What is necessary is an infrastructure to convert average available resources into vast instantaneous resources to conduct studies like these in a short time. This is achieved by integrating clusters into the Open Science Grid (OSG).

The OSG is a federation of research facilities and research groups created originally to facilitate analysis of data from the Large Hadron Collider (LHC). It now supports research in various disciplines. The GlueX collaboration and some of the computing resources of its member institutions are being incorporated into a virtual organization (VO). The $\mathrm{VO}$ presents both a set of resources that can be used by others and a set of computing needs to be satisfied by borrowing computing time opportunistically from other facilities. In this manner, there is a continuous exchange of resources that generally allows a higher use efficiency. Each computing facility on this grid is abstracted by a computing element (CE) and each data storage facility is abstracted as a storage element (SE). Typically these are services running on a collection of servers that manage the actual computation or storage on some subordinate set of resources. Running computing jobs on the OSG requires identification of useful resources, packaging the job in compliance with the policies of those resources and submitting the jobs to the corresponding CEs.

Identifying useful and available resources on the grid and ensuring compliance with each one can be a daunting task. A convenient abstraction of these various resources and constraints is offered by "glideinWMS" - the Glidein Based 
Workload Management System. Simply put, this system "brings" the resources to the user by reserving computing slots in the facilities that it tracks and presenting these to the client as virtual local computing resources. The computing slots which it locates in various facilities are amalgamated by a new batch processing system in a way that makes all the delivered resources appear more uniform in terms of local environment. Reservation of computing slots is performed by a glidein "factory" operated by the developers of this system. "Front ends" run on the client side to communicate the user's needs (i.e. local job queue) to the factory. An obvious advantage of this system is that jobs are matched dynamically with available resources, wherever they are found. By comparison, job submission directly to computing facilities involves a commitment to that queue: moving to a different queue is not possible.

Another important advance that facilitated this study is the gridmake system. An obstacle to running calculations on foreign computing facilities is the uncertain local software availability. Consider that this study requires a large stack of GlueX software, which further depends on several other general-purpose packages. Additionally, an analyst has some private code continuously under development. Computing facilities that are not associated with the GlueX effort would not have this software installed. Thus, one needs to assess a facility's environment and prepare it for running GlueX jobs. Furthermore, as outlined above, an analysis project like this requires several computing steps (e.g. simulation, reconstruction) that may not always be carried through in a chain to the end. Instead, it is more convenient for the physicist to set up a set of dependencies between the steps and allow the missing data components to be built as necessary. The gridmake package developed in our group seeks to mimic the dependency-based "make" utility 
to generate the necessary results on the grid. A set of [software] packages and processors are specified by the user for the gridmake utility with the appropriate dependencies between them. Packages are defined by the URL of their installation source while processes are defined by the commands to be executed to generate some data. The gridmake system also orchestrates the delivery of any input and output files to and from the designated storage resource manager (SRM), in this case, one of the GlueX data repositories. While this happens on the execution computer, the server side of gridmake coordinates the distribution of packages. This centralized service is necessary to keep track of installations on the grid: preventing the waste of multiple simultaneous installations in the same facility and collecting the installation logs to track the success of facilities' preparation.

\subsection{Signal Reconstruction and Analysis}

Analysis of events begins with ordering reconstruction of the raw event tracks and showers. This is performed by reconstruction classes in the GlueX software stack. (These are undergoing development while their progress is benchmarked in MC analyses such as this one.) Reconstructed tracks and showers are delivered with one or more hypotheses in each as to the identity of the particle. The analysis software designed for this work filters these hypotheses based on the criteria described below and then parses this revised hypothesis list recursively to assemble all possible event renditions consistent with the $b_{1} \pi$ final state. These renditions are culled based on the calculated intermediate state invariant mass examined with various constraints on the event kinematics. A final kinematic fit $^{12}$ is then performed on each of the remaining renditions and a confidence level

\footnotetext{
12 This is a higher level fit, in which the kinematics of the individual reconstructed particles are adjusted in light of their error bars in order to meet several constraints. At a minimum, the
} 
(CL) is formed from the fit results. The analysis software can then select the best rendition based on this parameter or keep all that conform to a minimum CL requirement. The following sections will describe these steps in greater detail.

\subsection{1 $\pi^{0}$ Reconstruction}

The $\pi^{0}$, with a lifetime of $8.4 \times 10^{-17} \mathrm{~s}$, can only be reconstructed indirectly through its daughter particles; the dominant (98.8\%) decay mode is $2 \gamma .[32]$ Accurate $\pi^{0}$ reconstruction requires proper identification of photon showers in the calorimeters and a good analysis filter for the combinatorial background that arises from incorrect photon pairing. The former is still a challenge at this stage of GlueX software development due to the state of the energy cluster association algorithms.

The mean free path of a hadron in a calorimeter is longer than that of a photon, resulting in deeper initial hadronic showers and "splitoffs" - secondary showers from a hadronic daughter of the incident hadron. The challenge for the reconstruction algorithms is distinguishing stand-alone showers from splitoffs and properly associating the latter to their parent shower. At the time of this analysis, the algorithms used in simulated GlueX reconstruction frequently failed to associate a separated shower and then identified it as a separate photon shower. This effect multiplied the number of possible photon combinations and significantly increased the background in $\pi^{0}$ reconstruction (Figure 4.2.) An additional problem that contributes to combinatorial background is noise in the BCAL. The current model for simulating noise hits in the BCAL is not realistic, nor are the algorithms properly tuned for suppressing realistic noise hits. Prior experience with calorime4 constraints (4C) of energy and momentum conservation is imposed. 
try indicates that a proper algorithm will be able to suppress most identifications of noise as showers and the effect of noise should contribute a negligible amount to systematic shifts in timing and energy. Thus, it was decided for the purpose of this study, to turn off the simulation of added noise in the BCAL [25].

Showers in the BCAL are associated with nearby charged tracks based on a maximum distance parameter in $z$ and $\phi$. The amended algorithm used for this study rescales this parameter by a factor of two when it considered inclusion of showers with no visible energy deposition in the innermost layer of the calorimeter. Showers with this signature are more likely to be hadronic, since an electromagnetically-showering particle coming from the target would begin losing energy soon upon entry. The remaining unassociated showers were then disqualified from being considered electromagnetic based on any of the following criteria:

1. shower's energy centroid deeper than $65 \%$ of BCAL module

2. energy in fourth layer (last, $40 \%$ of module thickness) is greater than $70 \%$ of total shower energy

3. significant gaps in showers between clusters: energy only in the first and fourth layers

4. all energy deposited in the first layer

These criteria and their thresholds were checked empirically by comparing simulated electromagnetic showers from $\pi^{0}$ photons and those from $\pi^{ \pm}$impacts. The right plot in Figure 4.2 shows the improved candidate $\pi^{0}$ spectrum from a standard Pythia event set, demonstrating clear improvement in background suppression. Further improvement in the signal to background ratio was gained at the analysis stage. 

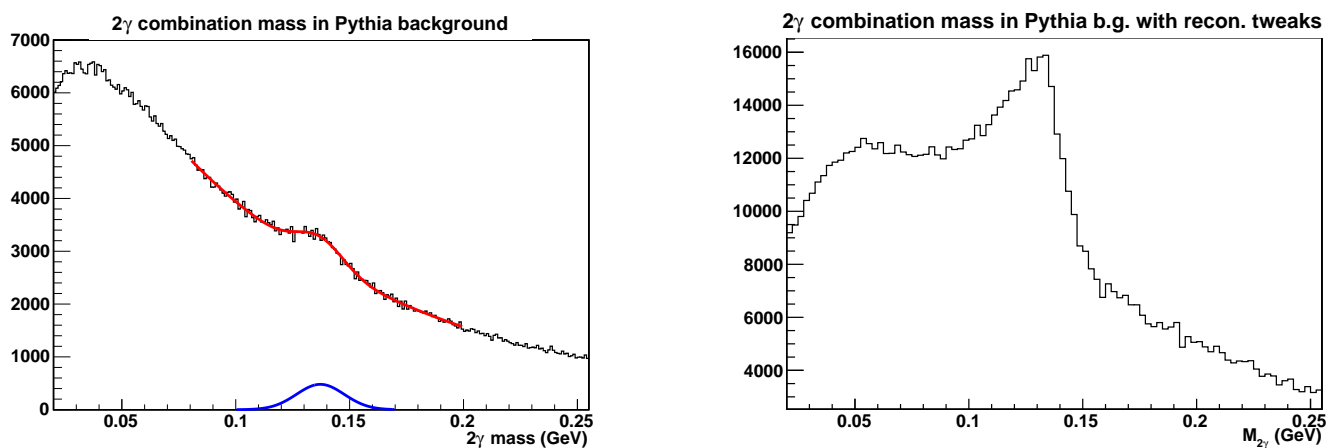

Fig. 4.2: Distribution of reconstructed invariant mass of $2 \gamma$ in a Pythia event sample. The left plot was produced using the base calorimetry algorithms and the current model of BCAL noise. The right plot was produced without BCAL noise hits and with tweaks to shower association and photon hypothesis identification algorithms.

A powerful selection criterion for photon showers is their timing. One can compare the measured time of flight time with that expected assuming speed of light and the measured distance. Figure 4.3 shows the distribution in this time difference for photons, $\pi^{+}$and protons using the raw reconstruction on a Pythia sample: no analysis-level particle identification revision or track fit quality cuts were made. (This means that the distribution of photon time deviations includes misidentified showers.) Based on this plot, a requirement was made that a photon shower hypothesis must have the measured time of flight within $8 \mathrm{~ns}$ of the expected. The right-hand plot in Figure 4.3 shows the improvement in the $2 \gamma$ mass spectrum: a significantly reduced background without significant reduction in the signal.

In the next step, a mass cut is imposed, keeping $2 \gamma$ candidate pairs within $40 \mathrm{MeV}$ of $\pi^{0}$ mass. The remaining pairs are now evaluated with respect to the full event. One $2 \gamma$ pair is selected and all possible event renditions are formed using the available set of charged track hypotheses (their filtering is discussed below.). 

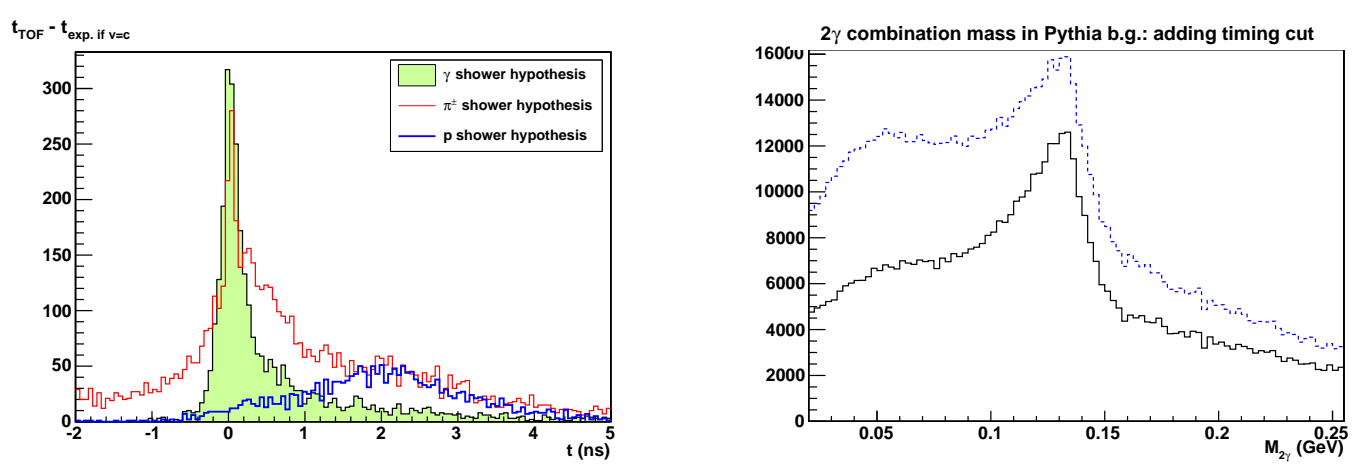

Fig. 4.3: Left: Distributions of the difference between the measured time of flight and that expected assuming speed of light. The distributions are taken from reconstruction without any analysis filters. (Note the enhancement in the $\pi^{+}$distribution due to misidentification of protons.) Right: The mass distribution that results from a \pm 8 ns photon timing cut (black solid line) is compared to the raw reconstructed distribution (blue/dash).

A kinematic "4C" fit (i.e. energy-momentum conservation only) is performed on all of these renditions to examine the resulting adjustments to the $2 \gamma$ pair mass. The pair is rejected if the best-fit rendition puts the mass beyond $30 \mathrm{MeV}$ of $\pi^{0}$ mass. If the pair survives this selection, it is subjected to a stricter kinematic fit: the candidate $\omega$ system mass is constrained. (This is very useful, since the $\omega$ has a very narrow width of $8.5 \mathrm{MeV}$ FWHM, offering a powerful event rendition selection tool.) The adjusted $2 \gamma$ mass is again evaluated with respect to the $\pm 30 \mathrm{MeV}$ requirement. The passing $2 \gamma$ is then fit to $\pi^{0}$ and the remaining kinematic fits on the event are made with this constraint.

\subsubsection{Charged Track Reconstruction and Identification}

It should be noted that at this point, the reconstruction software has a poor filter for incorrect charged track hypotheses. (At the time of this reconstruction study, the track fit confidence level was not reliably computed.) Specifically relevant 
for this study is the proliferation of proton and $\pi^{+}$hypotheses for each positive charged track. To distinguish these, positive tracks are examined based on energy loss $(d E / d x)$ and velocity determined from time of flight (TOF). Figure 4.4 shows the distribution of energy loss $(d E / d x)$ as a function of particle momentum for protons and pions in $b_{1} \pi$ events. Figure 4.5 shows the distribution of normalized particle velocity $\beta$ as a function of track energy. Both the $d E / d x$ and $\beta$ distributions at low energy are separate enough for a discrete cut to identify the particle identity (PID), but a more nuanced approach that helps resolve PID in overlap regions of these distributions is a $\chi^{2}$ test. Track parameters are tested by calculating the p-value with respect to the distributions of the two hypotheses. The identification with the best p-value is taken.
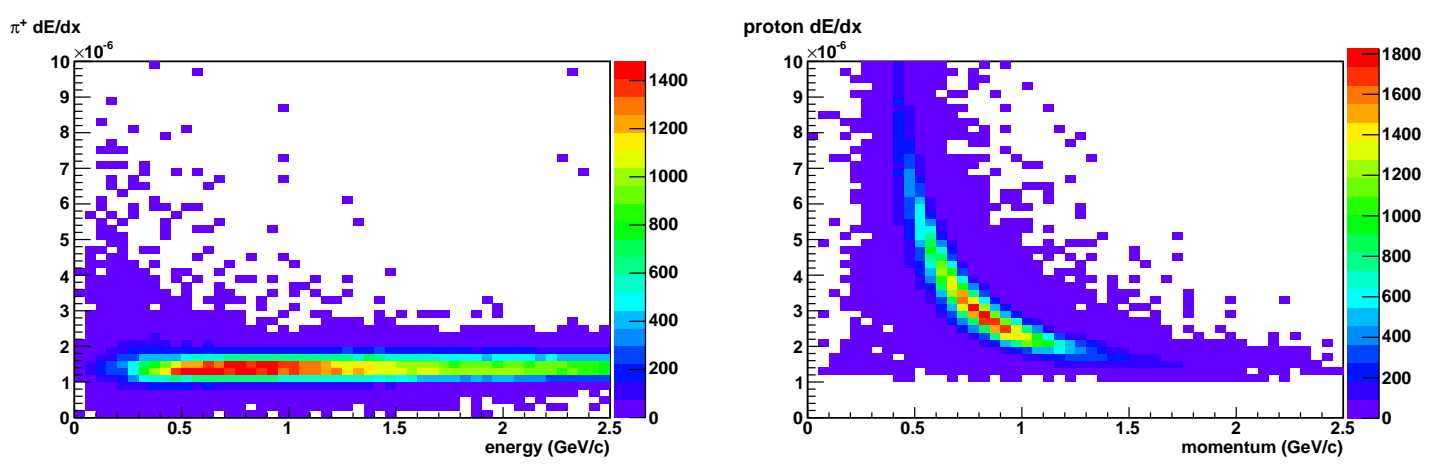

Fig. 4.4: Distributions of energy loss rates versus particle momentum magnitude for protons and $\pi^{+}$.

\subsubsection{Event Rendition Selection and Kinematic Fitting}

If the set of reconstructed particle hypotheses contains the basic constituents of the $b_{1} \pi$ final state: two $\pi^{+}$, two $\pi^{-}$, one proton, in addition to a viable $2 \gamma$ candidate for a $\pi^{0}$ (discussed above), the set is arranged into all possible event renditions. A key signature of this decay is an intermediate $\omega$ particle, which has 

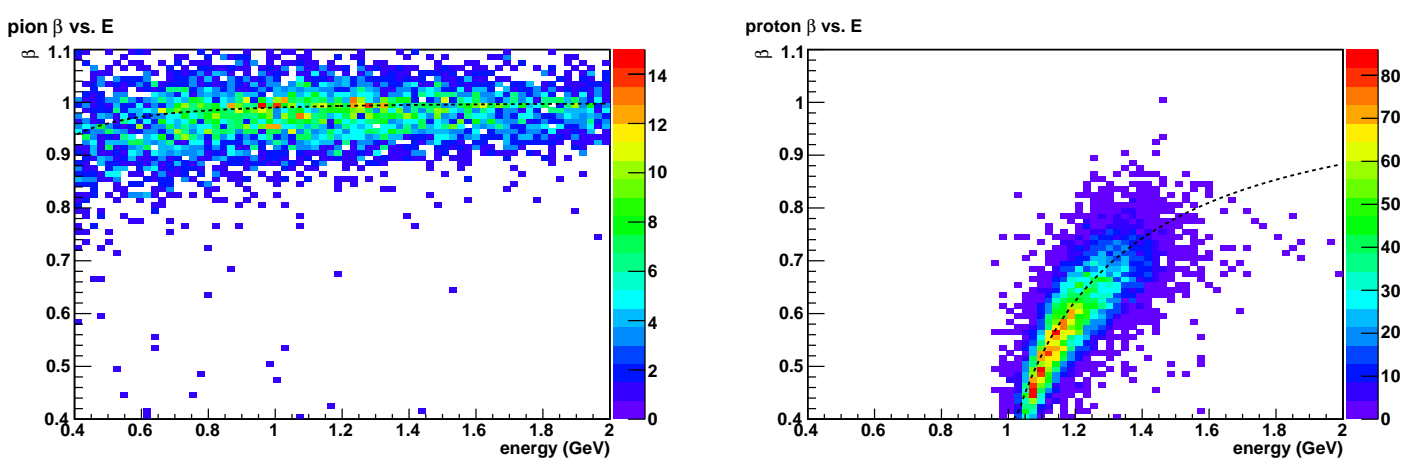

Fig. 4.5: Distributions of reconstructed normalized particle velocity $(\beta)$ versus energy for protons and pions. The expected dependence of velocity on energy is shown in dashed line.

a narrow width of $\sim 8.5 \mathrm{MeV}$. Identifying this feature is a key for selecting the correct rendition and selecting $b_{1} \pi$ events in the data stream in general. Using the already selected $2 \gamma$ pair for the $\pi^{0}$, all possible combinations of $\pi^{+}$and $\pi^{-}$are taken to form the candidate $\omega$ system (assuming only the dominant, $89 \%$ channel: $\omega \rightarrow \pi^{+} \pi^{-} \pi^{0}$ ) [32]. For each of these, all possible combinations for resonance and $b_{1}$ bachelor pions are taken. With only the $\pi^{0}$ mass constrained, a kinematic fit is performed on each of these renditions. A candidate $\omega$ is rejected if its fit-adjusted mass is outside $\pm 36 \mathrm{MeV}$ of the $\omega$ mass $(783 \mathrm{MeV})$. A surviving $\omega$ system triplet is then constrained to the $\omega$ mass in a final set of kinematic fits. (This is a "6C" fit at this point, with $\pi^{0}$ and $\omega$ system masses constrained.) A loose 0.001 final kinematic fit CL cut is imposed on the remaining renditions. The fit-adjusted kinematics of each event rendition that passes this cut are recorded, along with their event and track fit data. It is expected that further cuts are imposed after this basic analysis.

These procedures do not force all the tracks and showers to be incorporated into the event. It is assumed that there are often extra tracks or showers due to background or mis-reconstruction. (The latter is still a significant issue at this 
stage of GlueX software development.) However, to guard against contamination from competing physics processes with more prongs in the decay, a check on the remaining tracks is performed. A rendition that does not incorporate one or more tracks with a confidence level greater than $20 \%$ is excluded. As more progress is made on reconstruction and PID, a study of reconstructed signal purity as a function of this parameter would be useful and the threshold for this parameter ought to be reexamined.

So far, the analysis aimed at bare identification of events and their renditions that conform to $b_{1} \pi$ kinematics. In the second stage of analysis, further requirements are set to optimize the purity of the event sample with respect to other hadronic processes while trying to keep a high efficiency. At this point some obvious cut parameters still remain. The requirement of conformity to the $\omega \pi \pi$ kinematics can be tightened with a cut on the overall event confidence level. Conformity to $b_{1} \pi$ kinematics in particular can be enforced with the confidence level formed from the $b_{1}$ lineshape $\chi^{2}$.

\subsubsection{Suppression of Contamination from $\Delta$ resonances}

One of the significant sources of background is the presence of $\Delta$ resonances in $5 \pi$ events. The target proton may be excited into a $\Delta(1232)$ resonance which decays to $p \pi$ while the photon may produce some four or more pions. This type of events can be suppressed in a few ways with various costs to the signal reconstruction efficiency. One can form hypotheses for a $\Delta$ resonance by looking at protonpion pairs. Since we expect peripherally-produced pions to be more forward, we identify the lowest mass $p \pi$ system as potentially a $\Delta$ decay. Selection can then be made based on this invariant mass or the decay angles of this system. The 
distributions of these quantities are shown in Figures 4.6 and 4.7. The plot of the $p \pi$ system invariant mass distribution makes it clear that there is no clear forward-backward system separation: many known peripherally-produced pions form a very low mass system with the proton.
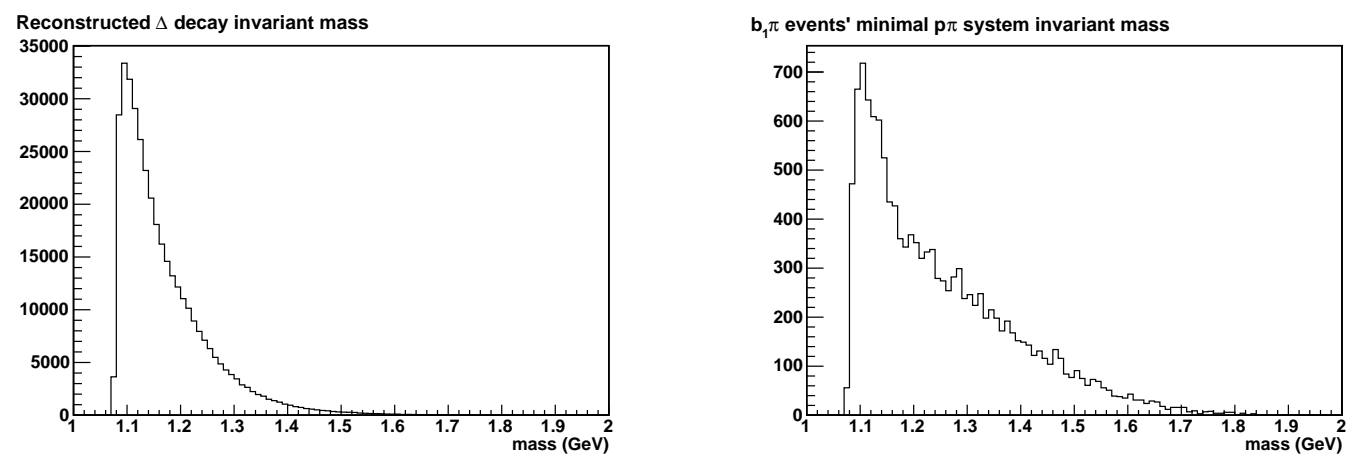

Fig. 4.6: Left: Reconstructed $\Delta$ invariant mass in known events with $\Delta$ resonances. Right: $p \pi$ system invariant mass in $b_{1} \pi$ events selected for minimal mass. It is clear that there is no forward-backward separation that allows any convenient cut.

Angular distributions of a potential $\Delta$ system offer another set of selection parameters.

The decay $\Delta \rightarrow p \pi$ should have a P-wave distribution. This is not modeled accurately in Pythia, where the angular distribution of the daughter particles is uniformly distributed. Nonetheless, comparison of this angular distribution and that from false pion-proton pairing offers a cut that will be useful in the presence of properly-distributed $\Delta$ decays. A system assembled from a false $p-\pi$ pairing, the polar angle, defined as the direction of the proton in the $\Delta$ rest frame, is expected to be dominantly backward when paired with a forward pion. The azimuthal angle would also contain an artifact of false $\Delta$ identification. To transform to the rest frame, the system is rotated to $\phi=0, \theta=0$ followed by a boost along the $z$-axis. The consequence of false proton-pion pairing is a pileup near $\phi=0$. A 
comparison of Pythia's $\Delta$ decay distributions, those determined in reconstruction and those reconstructed from false $\Delta$ decay hypotheses is shown in Figure 4.7. The plots indeed show that the more uniform reconstructed real $\Delta$ decay distributions offer a background filter with some cost to the signal reconstruction efficiency. Selection of optimal thresholds on these parameters is left to a later optimization procedure. Proper modeling of the $\Delta$ decay ought to be performed at a more advanced stage of this work to understand the selection power between real and false $\Delta$ system identification.
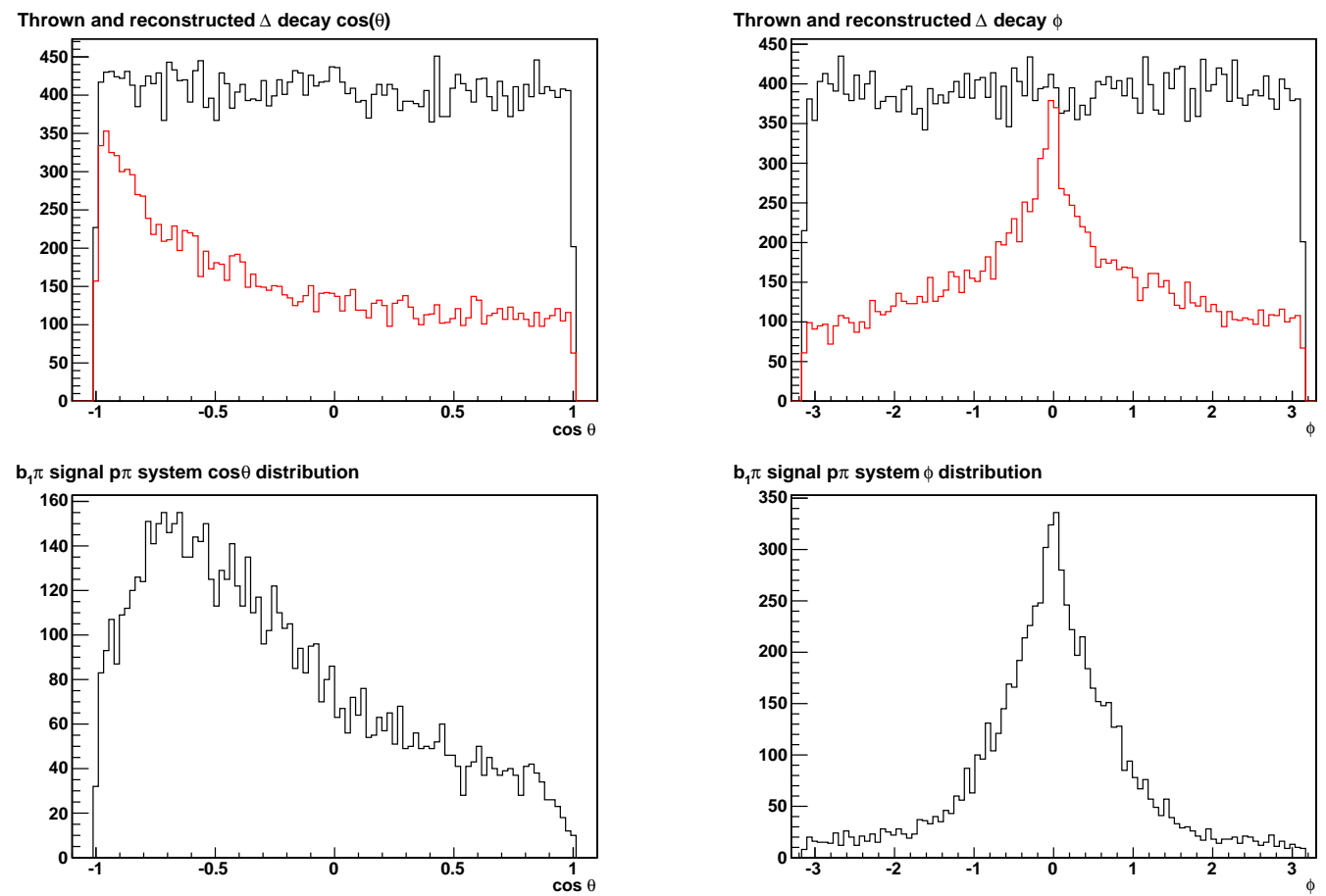

Fig. 4.7: The upper row of plots shows the angular distributions of the decay of $\Delta$ resonances in the Pythia dataset as well as the reconstructed angular distribution. Below are the distributions of $p \pi$ system in the $b_{1} \pi$ signal data. (The event reconstruction software identifies the lowest mass $p \pi$ system as a candidate $\Delta$ and forms these quantities.) The uniform distribution of real $\Delta$ decays compared to the peaked distributions of false $\Delta$ systems offers a chance at cuts to improve signal purity. 


\subsubsection{Cut Threshold Optimization}

The analysis procedures above calculated various parameters that may be useful for reducing contamination of a reconstructed event sample. These include confidence levels on the $\pi^{0}$ fit, overall event kinematic fit, $b_{1}$ lineshape, proton $d E / d x^{13}$, and the parameters of a suspected $\Delta$ system: $\theta_{\Delta}, \phi_{\Delta}$ and $m_{\Delta}$. It is useful to examine how the thresholds on these parameters effect the signal reconstruction efficiency and purity. In general, one prefers the maximal purity. However, with a limited statistical sample, a compromise is necessary between efficiency and purity. So, it may be useful to study various combinations of cuts to determine a permissible level of background in case of a limited signal sample.

A reconstructed event selection was prepared from $b_{1} \pi$ event Monte Carlo as "signal". For background, a Pythia event set was prepared with $\omega \pi \pi$ events excluded (i.e. not considered "background"). A Multi-Objective evolutionary optimization was performed to find a range of solutions (purity/efficiency compromises) and their corresponding cuts. This procedure evaluates the objective functions: signal efficiency and purity of the given event sample in terms of the cut thresholds. This two-objective optimization requires that all peer solutions are kept. Two solutions are defined as peers if one does not have all of its objective parameters inferior to those of the other. In the course of this optimization, new solutions are found that are superior in both objective parameters to an older solution. The latter is then discarded. Figure 4.8 shows the Pareto-optimal front in the objective space, representing the presumed contour of efficiency/purity compromises possible with the given cut parameters.

\footnotetext{
${ }^{13}$ Although the proton and $\pi^{+}$hypotheses were already scrutinized using $\chi^{2}$ tests, a stricter rectangular cut in the $d E / d x$ overlap region of these particles $\left(p>>m_{\text {proton }}\right)$ is still possible. Thus we allow ourselves to set a minimum proton $d E / d x$ threshold in the second stage of the analysis.
} 


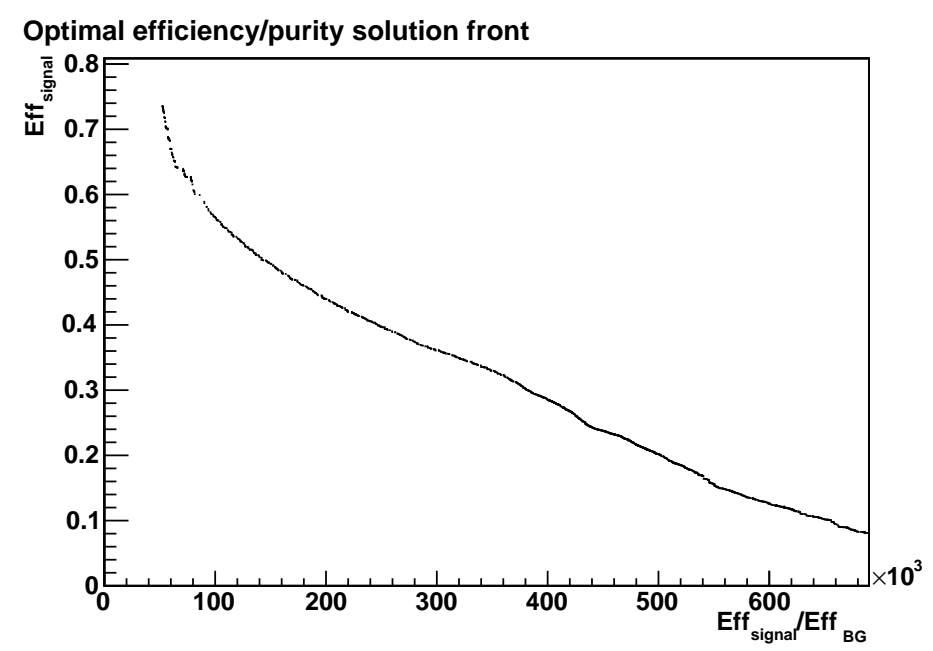

Fig. 4.8: The Pareto-optimal front of solutions optimizing efficiency and purity of a data sample given the available cut parameters. The units are not meaningful in themselves as they scale from rates and efficiencies of a baseline set of cuts.

\subsection{Fitting (Simulated) Data: Maximum Likelihood Method}

The number of degrees of freedom taken into account in Amplitude Analysis modeling is truly daunting. In this channel, for example, we have five final state particles and the recoil proton with a known mass for each. With three remaining degrees of freedom per particle, each event can be described by a point in an 18-dimensional space! A common binned fit in such a space must be abandoned in favor of an un-binned approach like the Maximum Likelihood (ML) method.

The "likelihood" is constructed as the combined probability for detecting all the events seen and getting the detected count of events assuming random arrival. Thus we take the product of individual event probabilities and the Poisson probability density distribution

$$
\mathcal{L}=\frac{e^{-\mu} \mu^{N}}{N !} \prod_{i}^{N} P\left(\overrightarrow{x_{i}}, \vec{\theta}\right)
$$


where $N$ is the observed number of events, $\mu$ is the expected number and $P\left(\overrightarrow{x_{i}}, \vec{\theta}\right)$ is the probability for observing the event $i$ with kinematics of the configuration $\overrightarrow{x_{i}}$, with the probability function parameters $\vec{\theta}$. The expected number of events may be expressed as a hypothetical quantum mechanical probability (intensity) $I$ weighted by the detector acceptance $\eta$. Thus the expected number of events detected may be expressed as

$$
\mu=\int \eta(\vec{x}) I(\vec{x}, \vec{\theta}) d \vec{x} \approx \frac{1}{N_{\text {gen }}} \sum_{i}^{N_{\text {acc }}} \eta\left(\overrightarrow{x_{i}}\right) I\left(\overrightarrow{x_{i}}, \vec{\theta}\right)=\frac{1}{N_{\text {gen }}} \sum_{i}^{N_{\text {acc }}} I\left(\overrightarrow{x_{i}}, \vec{\theta}\right)
$$

where the integral over all kinematic variables in this type of an event with a smooth acceptance function $\eta(\vec{x})$ can be approximated by the Monte Carlo method, resulting in a sum over the calculated event intensities. Now, the function $\eta$ becomes trivial, evaluating to 1 for every event seen and 0 for every undetected event. Since $\overrightarrow{x_{i}}$ refers to the kinematics of an observed event, $\eta$ is 1 for all terms. The total number of events thrown $\left(N_{\text {gen }}\right)$ is used for normalization. Now, we can define the hypothesis probability for the i-th event using the expected number of events $\mu$ for normalization.

$$
P\left(\overrightarrow{x_{i}}, \vec{\theta}\right)=\frac{I\left(\overrightarrow{x_{i}}, \vec{\theta}\right)}{\mu}
$$

Expanding the intensity in terms of $n$ coherent quantum mechanical amplitudes:

$$
I\left(\overrightarrow{x_{i}}, \vec{\theta}\right)=\sum_{\gamma, \delta} \rho_{\gamma \delta} \sum_{\alpha, \beta}^{n} u_{\alpha} u_{\beta}^{*} A_{\alpha}^{\gamma}\left(x_{i}, \vec{b}\right) A_{\beta}^{\delta *}\left(x_{i}, \vec{b}\right)
$$

where the new variables $\vec{b}$ and $u$ are the free amplitude parameters and coefficient respectively, previously represented by the parameters $\vec{\theta}$, and $\rho$ is the spin den- 
sity matrix for the beam photon. Note the important distinction between these variables with respect to the computational complexity of an amplitude fit: if the amplitude is parametrized - depends on parameters other than event kinematics - the amplitude of an event must be recomputed for any change in parameters during fitting. However, if only coefficients $u_{\alpha}$ vary, altering the mixture of waves, the event amplitudes need only be computed once.

It is computationally more convenient to deal with the logarithm of the likelihood, turning a product into a sum. Combining the above expressions yields

$$
\begin{aligned}
\log \mathcal{L} & =\log \left[\frac{e^{-\mu}}{N !} \prod_{i}^{N} I\left(\overrightarrow{x_{i}}, \vec{\theta}\right)\right]=-\log (N !)-\mu+\sum_{i}^{N} \log \left[I\left(\overrightarrow{x_{i}}, \vec{\theta}\right)\right] \\
& =C-\frac{1}{N_{\text {gen }}} \sum_{i}^{N_{\text {acc }}} \sum_{\gamma, \delta} \rho_{\gamma \delta} \sum_{\alpha, \beta}^{n} u_{\alpha} u_{\beta}^{*} A_{\alpha}^{\gamma}\left(x_{i}, \vec{b}\right) A_{\beta}^{\delta *}\left(x_{i}, \vec{b}\right) \\
& +\sum_{i}^{N} \log \left[\sum_{\gamma, \delta} \rho_{\gamma \delta} \sum_{\alpha, \beta}^{n} u_{\alpha} u_{\beta}^{*} A_{\alpha}^{\gamma}\left(x_{i}, \vec{b}\right) A_{\beta}^{\delta *}\left(x_{i}, \vec{b}\right)\right] \\
& =C-\sum_{\alpha, \beta}^{n} u_{\alpha} u_{\beta}^{*}\left[\frac{1}{N_{\text {gen }}} \sum_{i}^{N_{\text {acc }}} \sum_{\gamma, \delta} \rho_{\gamma \delta} A_{\alpha}^{\gamma}\left(\overrightarrow{x_{i}}, \vec{b}\right) A_{\beta}^{\delta *}\left(\overrightarrow{x_{i}}, \vec{b}\right)\right] \\
& +\sum_{i}^{N} \log \left[\sum_{\gamma, \delta} \rho_{\gamma \delta} \sum_{\alpha, \beta}^{n} u_{\alpha} u_{\beta}^{*} A_{\alpha}^{\gamma}\left(\overrightarrow{x_{i}}, \vec{b}\right) A_{\beta}^{\delta *}\left(\overrightarrow{x_{i}}, \vec{b}\right)\right]
\end{aligned}
$$

where $C$ is the constant term which may be neglected in log-likelihood optimiza-

tion. Note that for fixed amplitude parameters $\vec{b}$, the matrices of event amplitude products indexed by $\alpha, \beta$ may be pre-computed.

\subsection{Fit Results}

A $b_{1} \pi$ signal was generated of about 4.5 million events with the waves listed in Table 4.2 . 
Table 4.2: Input waves for the decay $X \rightarrow b_{1} \pi$

\begin{tabular}{ccccc}
\hline \hline wave $\left(J^{P C}\right)$ & $L$ & $S$ & $m_{0}(\mathrm{GeV})$ & $\Gamma_{0}(\mathrm{GeV})$ \\
\hline $1^{--}$ & 0 & 1 & 1.89 & 0.16 \\
$2^{+-}$ & 1 & 1 & 2.00 & 0.25 \\
\hline
\end{tabular}

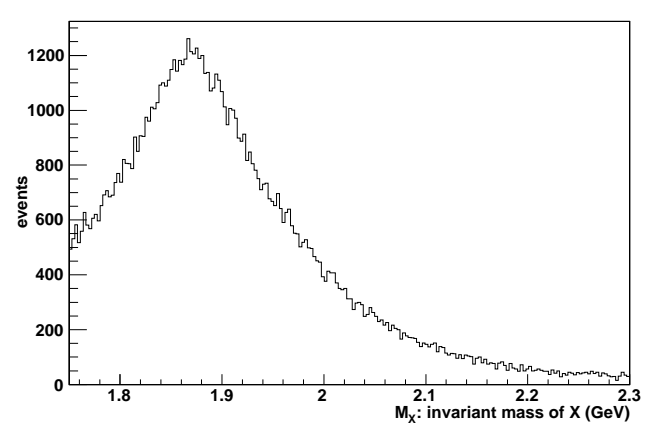

Fig. 4.9: Generated $b_{1} \pi$ invariant mass distribution using a mixture of $1^{--}$and $2^{+-}$waves.

The relative scaling of the exotic $2^{+-}$to the non-exotic $1^{--}$was $0.86 .40 \%$ polarization and a mono-energetic photon beam was assumed. Another simplifying assumption made for this study is perfect knowledge of the energy (i.e. no ambiguity due to tagger accidentals.) Figure 4.9 shows the resulting invariant mass distribution of the generated resonances.

Positive reflectivity eigenvalues of the Reggeon $\left(\epsilon_{R}\right)$ were taken for generating the signals. False waves included in the fits described below included amplitudes with the same $J^{P C}$ quantum numbers and negative reflectivity states, as well as $1^{++}$and $2^{++}$waves in each of the allowed values of $\epsilon_{R}$.

Before proceeding with simulating this physics sample in the GlueX detector, a check on the generation and fitting software was made. The generated physics Monte Carlo sample along with the generated phase space event sample were fed directly into the fitting procedure. This is equivalent to assuming isotropic, $100 \%$ acceptance for all kinematics and perfect resolution. Figure 4.10 displays the 
results of this study, plotting the invariant mass distribution (histogram) and the squared production amplitude coefficient values superimposed as colored points. The participating coherent waves are plotted (red and green) by squaring their individual amplitudes. ${ }^{14}$ The fit results can be compared to the expected curves (gray bands.) First, a note is in order about how these expected curves are calculated.

The squared amplitude functions studied here have no simple expression in terms of the invariant mass. For any value of the invariant mass, there exists a phase space of daughter particle kinematics. To compute these functions, therefore, Monte Carlo sampling is made of this phase space, weighted by the known amplitude dependence. What is calculated, then, is an approximation to the true values with an uncertainty (width of the drawn band) appropriate to a finite MC sample. In other words, a new MC sample is made analogously to what was done to generate the signal. Now, this is repeated for the total coherent mixture as well for each amplitude by itself.

Figure 4.10 also shows the phase difference between the two coherent waves. Two models are used to compute the expected phase differences as a function of mass. A simple "naive" model uses two simplified Breit Wigners with the true input parameters. These do not depend on the breakup momentum of the daughter particles. Results from a more sophisticated model are also shown, in which every point derives from an event from the signal MC generator. The relative phase of the amplitudes for each thrown event is plotted. No weighted average over the phase space of the daughters is possible because this would be

\footnotetext{
${ }^{14}$ In other words, these do not truly represent the resonance shape of the individual amplitudes but rather show a related plot of what the distributions would be if the amplitudes did not mix coherently. Therefore, it should not be expected that the plotted functions (blue and green) add to the overall fit of resonance mass (red.)
} 
equivalent of coherent summation over independently measured events. Only the calculation of relative phases of amplitudes within one event is meaningful.

Figure 4.10 suggests that the fit results follow the expected as a function of invariant mass. However, the reduced $\chi^{2}$ computed to determine the goodness of fit is 2.6 and 8.8 for the $1^{--}$and $2^{+-}$, respectively. This indicates that the fits may not have converged to the absolute minimum. This was indeed suggested by the MINUIT's MIGRAD fitting algorithm with warnings that it failed to find improvement when the given machine accuracy was reached $\left(10^{-13}\right.$ in these studies). Further work is necessary to study the systematics of convergence and understand whether all contributions to error were taken into account, whether the fitting framework estimated the errors properly.

Note also the large point-to-point fluctuations in the fitted phase difference plot relative to the error bars. The calculated errors in the limit of high statistics are smaller than the observed mean value variation of adjacent points. This could indicate a systematic effect in the way it is calculated or may present further evidence of poor convergence of the fit (as was suggested in the invariant mass projection).

Figure 4.11 shows fit results and expected values from the same procedures as above but with detector acceptance and resolution taken into account. The MC-generated physics events were simulated in HDGeant - the GlueX detector MC simulation. GlueX reconstruction is performed with the resulting hits in the virtual detector, followed by the analysis and cuts described above. The figure shows an overall broadening of the resonance invariant mass and a small amount of leakage into the uniform wave. Comparing to the expected values in the upper plot, it appears that the leakage is primarily from the $1^{--}$wave. The right-hand 

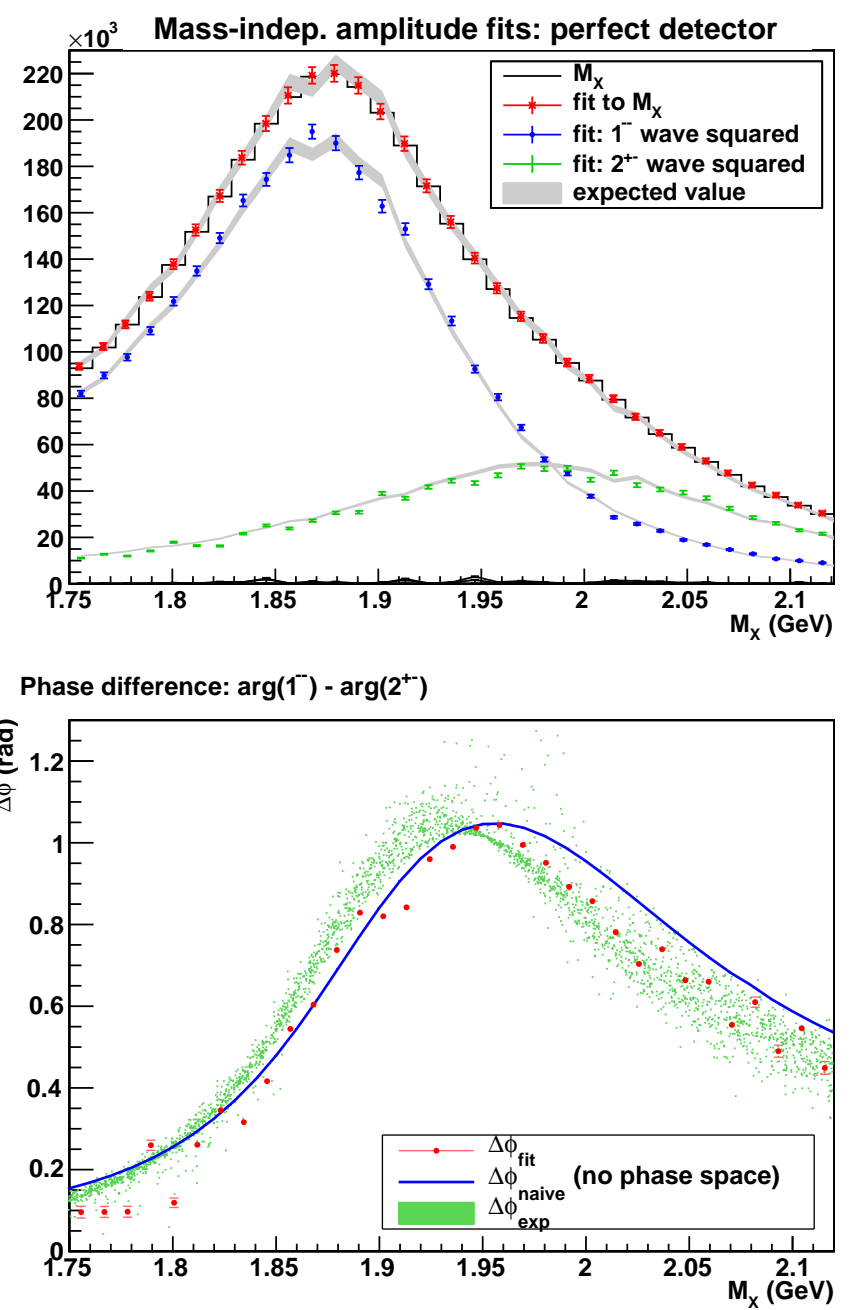

Fig. 4.10: Top: Results of a mass-independent fit of a $b_{1} \pi$ signal sample, assuming a detector of perfect acceptance and resolution. Negligible leakage (dark curves at the bottom) to false waves is seen. Bottom: The phase difference between the two coherent waves is shown, with the expected values computed in two ways: "naive" phase difference between two Breit Wigners without breakup momentum in the resonance's two-body decay and a scatter of phase differences for each thrown event.

plot in the figure compares the fit and expected values when the latter are rescaled to account for this leakage.

The relative phase between the $1^{--}$and the $2^{+-}$waves is plotted at the 
bottom of Figure 4.11 in the same way as above. The error bars are included as guides to the level of fit uncertainty, but it is still apparent that these uncertainties are inconsistent with point-to-point variations, which indicates poor fit convergence or some other systematic effect not taken into account in the plotted uncertainty.
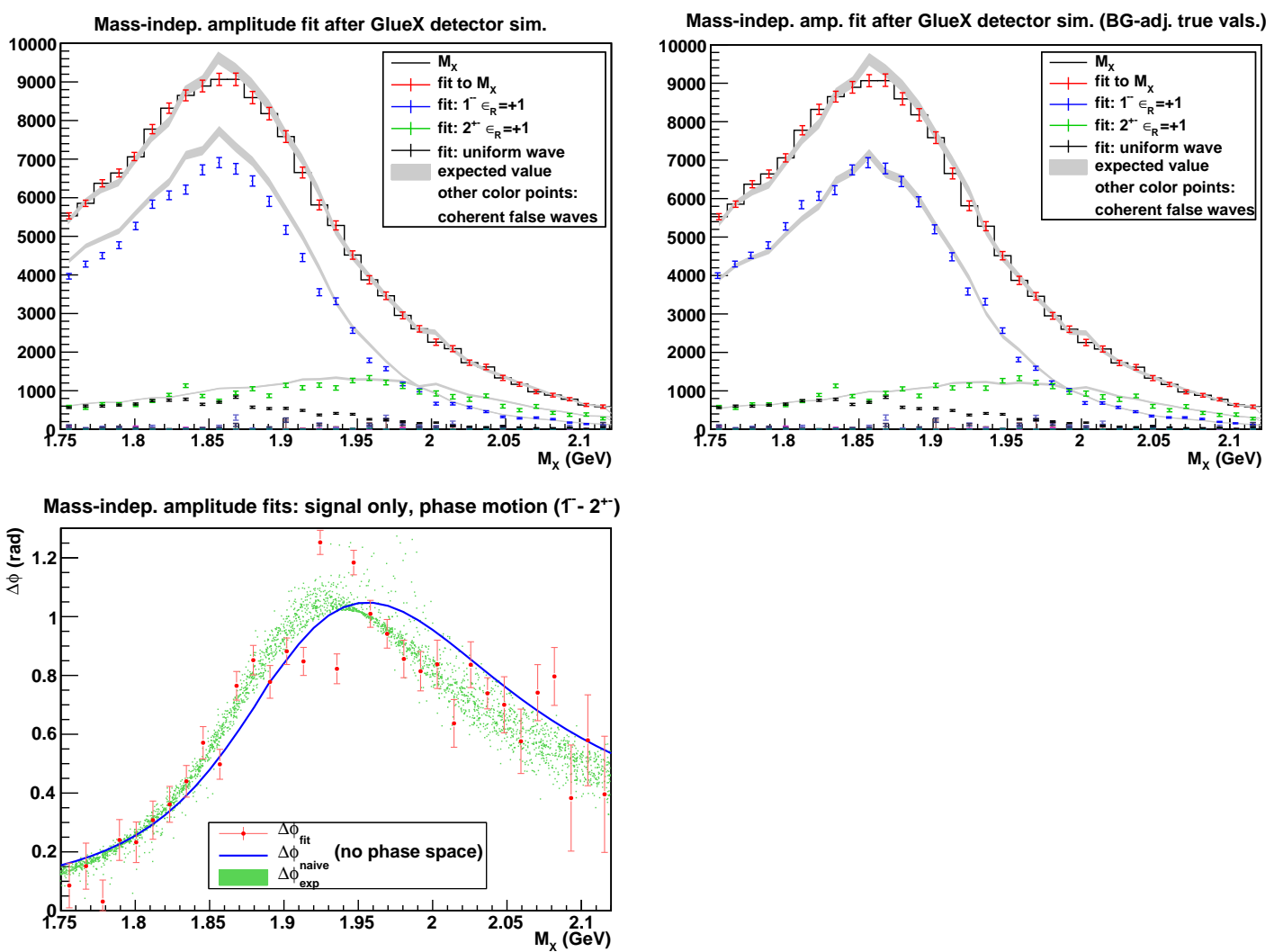

Fig. 4.11: Top-left: Results of a mass-independent fit with $b_{1} \pi$ signal events passing GlueX reconstruction. Leakage mainly from the $1^{--}$to the uniform wave is apparent. Same plot with expected values, rescaled to account for this leakage, is shown in the top-right. Bottom-left: The phase difference between the two coherent waves is shown with error bars included as indicators of fit uncertainty. Accuracy of fit convergence and perhaps other systematic contributions to the error bars are not fully understood. The expected values are computed in two ways: "naive" phase difference between two Breit Wigners without breakup momentum in the resonance's two-body decay and a scatter of phase differences for each thrown event. 
Figure 4.12 shows the results from an analogous fit exercise with more physics signals included. Aside from the resonant mixture of $1^{--}$and $2^{+-}$waves, Pythia's mixture of reactions in photo-production is included. Among these is a non-resonant $\omega \pi \pi$ signal channel. The figure separates this channel from the Pythia contribution, as well as events known to come from non- $\omega \pi \pi 5 \pi$ final state, and events with a $\Delta$ resonance. This separation helps understand the effect of the above-discussed cuts on signal contamination and the effect on the fit results. The mixture of $b_{1} \pi$ signal with the Pythia event set is scaled such that the $2^{+-}$ resonance has an approximate cross-section (if taken by itself) of $40 \mathrm{nb}$ and the total photo-production cross-section is at the known value of $120 \mu \mathrm{b} .{ }^{15}$ A total of $13.9 \times 10^{9}$ Pythia events were simulated for this study, equivalent to about $260 \mathrm{~h}$ of run time assuming a rate of $15 \mathrm{kHz}$ from the $\mathrm{CB} 9 \mathrm{GeV}$ peak.

Results in Figure 4.12 show that the fit values roughly follow the expected curves. They also indicate a significant leakage from the uniform wave, which should accurately describe the decays to $\omega \pi \pi$ generated by Pythia. This is shown more clearly in the log plot of the same results in Figure 4.13. This figure also includes a fit to the $\omega \pi \pi$ Pythia channel alone. The uniform wave is expected to fit the histogram of $\omega \pi \pi$ events by itself because these events are thrown with a uniform angular distribution. What Figure 4.13 shows is that a significant fraction of the expected uniform wave strength leaks into other waves, including the exotic $2^{+-}$. This may be a source of ambiguity in a real search for an exotic state.

As with previous results, the level of fluctuation of data points from the fit in both the invariant mass and phase projections are inconsistent with the error bars. As mentioned earlier, more work is needed to investigate fit convergence

\footnotetext{
${ }^{15}$ Recall that a $9 \mathrm{GeV}$ mono-energetic beam is assumed in this study. Thus a constant value of $120 \mu \mathrm{b}$ is used.
} 
and other possible systematic effects. However, these results show a promising start in an the effort to build analysis procedures for the future GlueX data. 
Mass-indep. amplitude fits: $\sim 40 \mathrm{nb}$ signal
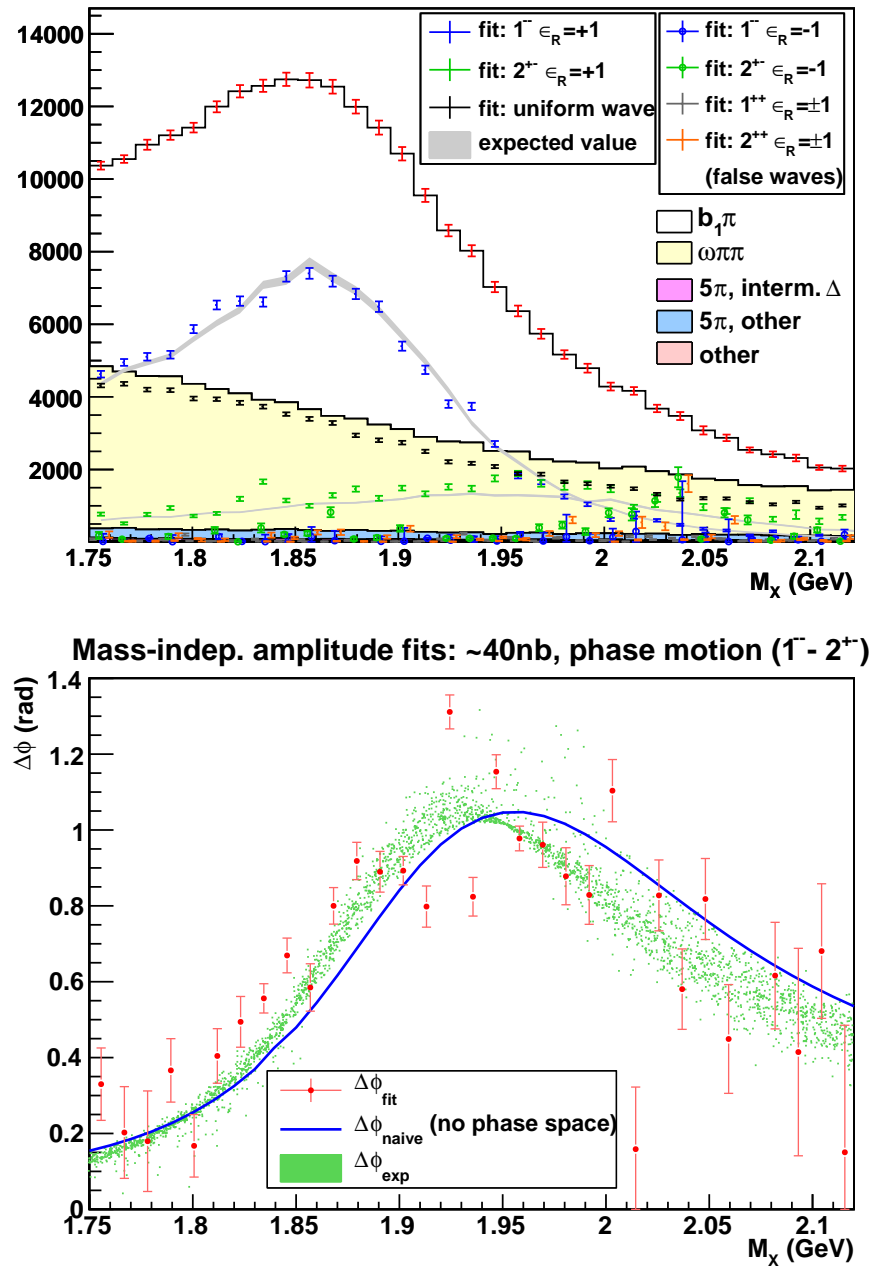

Fig. 4.12: Top: Results of a mass-independent fit with the events passing GlueX reconstruction. The generated $b_{1} \pi$ signal with a $40 \mathrm{nb}$ exotic $2^{+-}$ and the $120 \mathrm{nb}$ photo-production physics samples are included. Some leakage of Pythia's $\omega \pi \pi$ signal into the exotic $2^{+-}$and other waves is apparent. Greater leakage at higher invariant mass is also visible, likely due to lower acceptance MC statistics in that limit. Bottom: The phase difference between the two coherent waves is shown with error bars included as indicators of fit uncertainty. Accuracy of fit convergence and perhaps other systematic contributions to the error bars are not fully understood. The expected values are computed in two ways: "naive" phase difference between two Breit Wigners without breakup momentum in the resonance's two-body decay and a scatter of phase differences for each thrown event. 
Mass-indep. amplitude fits: $\sim 40 \mathrm{nb}$ signal
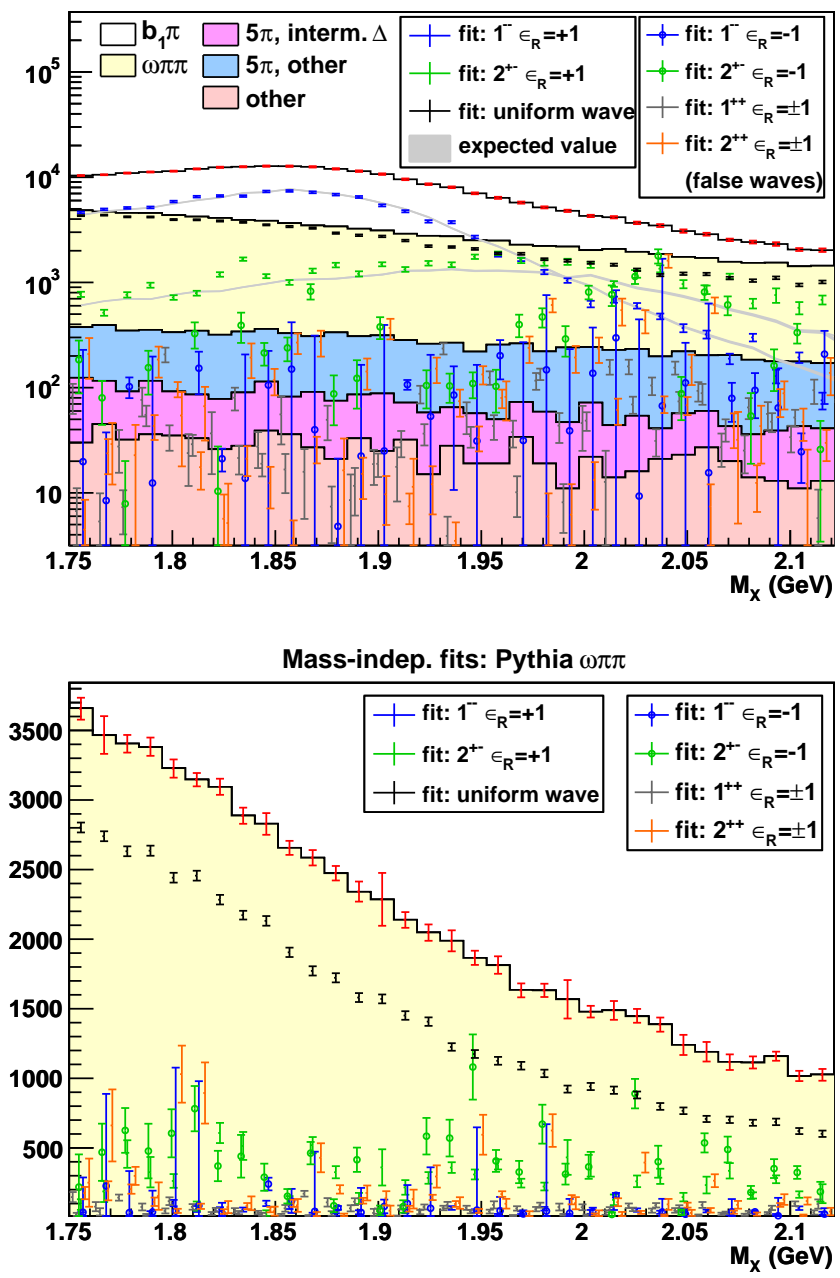

Fig. 4.13: The top plot shows the same results as in Figure 4.12 on the log scale for a clearer view of the leakage to the false waves. The bottom plot shows the results of a fit to the $\omega \pi \pi$ Pythia channel alone. The decays in this channel are modeled with uniform angular distributions and should, therefore, be absorbed by the uniform wave. Note the leakage to other waves, including the exotic $2^{+-}$. This may be due to the artificial structures in the angular distributions that accumulate from instances of incorrect event reconstruction. 


\subsection{Conclusion}

This analysis of a simulated decay of $\gamma p \rightarrow X p \rightarrow b_{1} \pi \rightarrow \omega \pi \pi \rightarrow 5 \pi$ was undertaken to exercise GlueX reconstruction, Amplitude Analysis techniques and to test the overall capability of measuring an expected exotic state near $2 \mathrm{GeV}$ invariant mass. Amplitude fits were performed with unbinned, maximum likelihood fitting with the AmpTools software framework.

As an early study of this kind, a few simplifying assumptions were made. A mono-energetic, $9 \mathrm{GeV}$ beam was assumed with perfect knowledge of this energy: no ambiguity in tagger hits were included. To bypass the currently ongoing work in modeling barrel calorimeter noise and developing shower reconstruction algorithms, the calorimeter was assumed to be noiseless and several temporary tweaks on shower association were made to suppress combinatoric background from spurious photon shower hypotheses. (The performance of this simulated system in this mode is thought to be consistent with the performance of such detectors once properly tuned and optimized.) The set of physics simplifications in this study included Pythia's assumption of isotropic two-body decays. The exotic $2^{+-}$signal was included coherently with just one wave, $1^{--}$. Thus, all physics other than these waves are modeled simply with an isotropic, uniform wave (constant amplitude for all kinematics).

Under these conditions, it was demonstrated that the present techniques do identify resonances along with their $J^{P C}$ quantum numbers in GlueX-reconstructed signals dominated by significant amount of background. Indeed, a $40 \mathrm{nb}$ signal was reconstructed and assigned to the correct quantum numbers, following approximately the true resonance line shape in the background of the total $120 \mu \mathrm{b}$ photo-production cross-section. This simulated study also allowed a decomposi- 
tion of the physics signals to understand the sensitivity of the fit to each segment of the event set. Reconstruction of the $X \rightarrow b_{1} \pi$ signal by itself led to leakage to the uniform wave of order $10 \%$ relative to the total event number. Reconstruction ambiguities of the Pythia's uniform $X \rightarrow \omega \pi \pi$ decays likely caused the observed leakage of about $20 \%$ from the expected uniform wave to several waves, including the exotic $2^{+-}$. These are the experimental issues that lead to false positives in searches such as the one attempted by the GlueX experiment.

Further work is necessary in this and other studies of simulated signals before the start of data-taking in the GlueX experiment. Convergence of the amplitude fits must be examined to understand the poor $\chi^{2}$ results in this study. If this alone is not sufficient to explain the scatter of points that is presently inconsistent with the error bars, the calculation of uncertainty must be examined for missing systematic or statistical effects.

Naturally, the improvement in the results of such studies ought to be gauged as the reconstruction algorithms are perfected. Further analysis of this channel should drop the simplification made to barrel calorimetry and begin examination of signals generated by the real photon beam spectrum. This work must then be repeated in the presence of tagger accidentals realistic to the expected tagger rates. The resulting ambiguities must be examined in light of signal reconstruction efficiency and purity as well as amplitude determination accuracy.

There is further opportunity to practice Amplitude Analysis with more advanced and challenging physics modeling. An exotic signal can be hypothesized to be smaller than $40 \mathrm{nb}$ in cross-section and smaller relative to the dominant coherent wave than what was assumed in this study. More coherent waves can be included in the signal and more realistic modeling of the background can be 
made (e.g. resonant phase motion in related channels and non-trivial angular distributions). A more challenging but an important step forward in sophistication of these analyses would be to move beyond the isobar model, which assumed 2body decays with no rescattering of final state particles. This simplification has theoretical inconsistencies, which ought to be cured with more advanced models. After the photon beam instrumentation effort discussed in this dissertation, the examination of the effects of polarization on this and other physics channels would be a compelling next step. Such a study could be carried out by amplitudeanalyzing signals from beams of various degrees of polarization. The dependence on polarization fraction of fit results that emerge from these analyses benchmarks the effectiveness of beam polarization as a tool in exotic meson searches. 


\section{Bibliography}

[1] Amptools, http://sourceforge.net/projects/amptools/.

[2] G.S. Adams et al., Observation of a new $J(P C)=1$-+ exotic state in the reaction $p i-p \rightarrow p i+p i-p i-p$ at $18 \mathrm{GeV} / c$, Phys.Rev.Lett. 81 (1998), 57605763.

[3] M. Alekseev et al., Observation of a $J^{* *} P C=1-+$ exotic resonance in diffractive dissociation of 190-GeV/c pi-into pi-pi-pi+, Phys.Rev.Lett. 104 (2010), 241803, 7 page, 3 figures/ version 2 gives some more details, data unchanged/ version 3 updated authors, text shortened, data unchanged.

[4] Ted Barnes, F.E. Close, F. de Viron, and J. Weyers, $Q$ anti-Q $G$ Hermaphrodite Mesons in the MIT Bag Model, Nucl.Phys. B224 (1983), 241.

[5] H. Bilokon, G. Bologna, F. Celani, B. D'Ettorre-Piazzoli, R. Falcioni, et al., Coherent Bremsstrahlung in Crystals as a Tool for Producing High-Energy Photon Beams to be Used in Photoproduction Experiemtns at CERN SPS, Nucl.Instrum.Meth. 204 (1983), 299.

[6] J.D. Bjorken, CURRENT ALGEBRA AT SMALL DISTANCES, Conf.Proc. C670717 (1967), 55-81.

[7] P. Buzhan, B. Dolgoshein, L. Filatov, A. Ilyin, V. Kantserov, et al., Silicon photomultiplier and its possible applications, Nucl.Instrum.Meth. A504 (2003), 48-52.

[8] R.N. Cahn and G. Goldhaber, THE EXPERIMENTAL FOUNDATIONS OF PARTICLE PHYSICS, (1989).

[9] Michael S. Chanowitz and Stephen R. Sharpe, Hybrids: Mixed States of Quarks and Gluons, Nucl.Phys. B222 (1983), 211.

[10] S.U. Chung, K. Danyo, R.W. Hackenburg, C. Olchanski, J.S. Suh, et al., Exotic and $q$ anti- $q$ resonances in the $p i+p i$ - pi- system produced in $p i$ - $p$ collisions at $18 \mathrm{GeV/c/,} \mathrm{Phys.Rev.} \mathrm{D65} \mathrm{(2002),} 072001$. 
[11] Suh Urk Chung, SPIN FORMALISMS, (1971).

[12] S.R. Cotanch and Felipe J. Llanes-Estrada, Relativistic many body approach to exotic and charmed hybrid mesons, Nucl.Phys. A689 (2001), 481-484.

[13] Stephen R. Cotanch, Ignacio J. General, and Ping Wang, QCD Coulomb Gauge Approach to Exotic Hadrons, Eur.Phys.J. A31 (2007), 656-661, 6 pages, 9 figures.

[14] Dmitri Diakonov, Foundations of the constituent quark model, Prog.Part.Nucl.Phys. 36 (1996), 1-18.

[15] Jozef J. Dudek, The lightest hybrid meson supermultiplet in QCD, Phys.Rev. D84 (2011), 074023.

[16] Jozef J. Dudek, Robert G. Edwards, Balint Joo, Michael J. Peardon, David G. Richards, et al., Isoscalar meson spectroscopy from lattice $Q C D$, Phys.Rev. D83 (2011), 111502.

[17] Jozef J. Dudek, Robert G. Edwards, Michael J. Peardon, David G. Richards, and Christopher E. Thomas, Highly excited and exotic meson spectrum from dynamical lattice QCD, Phys.Rev.Lett. 103 (2009), 262001.

[18] _ Toward the excited meson spectrum of dynamical QCD, Phys.Rev. D82 (2010), 034508.

[19] Christopher Gauthier and Richard Jones, Simulation of a position-sensitive tungsten pin-cushion detector, GlueX-doc-67-v1 (2003).

[20] Yu.P. Gouz et al., Study of the wave with $J(P C)=1-+$ in the partial wave analysis of eta-prime pi-, eta pi-, $f 1$ pi- and rho0 pi-systems produced in pi$N$ interactions at $p(p i-)=37-G e V / c$, AIP Conf.Proc. 272 (1993), 572-576.

[21] Boris Grube, Hadron Spectroscopy with COMPASS, (2011), 5 pages, 6 figures, proceedings of the '19th Particles and Nuclei International Conference' (PANIC11), MIT, Cambridge, MA, USA, July 24 - 29, 2011.

[22] Nathan Isgur, Richard Kokoski, and Jack E. Paton, Gluonic Excitations of Mesons: Why They Are Missing and Where to Find Them, Phys.Rev.Lett. 54 (1985), 869.

[23] Nathan Isgur and Jack E. Paton, A Flux Tube Model for Hadrons in QCD, Phys.Rev. D31 (1985), 2910.

[24] F. James, Monte-Carlo phase space, (1968), Microfiche at Fermilab.

[25] Richard Jones, personal communication, 2012. 
[26] Gordon L. Kane, MODERN ELEMENTARY PARTICLE PHYSICS, (1987).

[27] Vitaly D. Kovaltchouk, Zisis Papandreou, George J. Lolos, and Keith Wolbaum, Read-out system for a barrel calorimeter (gluex project), GlueX-doc69-v1 (2003).

[28] Joachim Kuhn et al., Exotic meson production in the f(1)(1285) pi- system observed in the reaction pi- $p \rightarrow$ eta $p i+p i-p i-p$ at $18 \mathrm{GeV} / c$, Phys.Lett. B595 (2004), 109-117.

[29] M. Lu et al., Exotic meson decay to omega pio pi-, Phys.Rev.Lett. 94 (2005), 032002 .

[30] C.A. Meyer and Y. Van Haarlem, The Status of Exotic-quantum-number Mesons, Phys.Rev. C82 (2010), 025208.

[31] Guthrie Miller and Dieter R. Walz, A tungsten pin cushion photon beam monitor, Nuclear Instruments and Methods 117 (1974), no. 1, 33 - 37.

[32] K. Nakamura et al., Review of particle physics, J.Phys.G G37 (2010), 075021.

[33] Stephan Narison, Gluonia, scalar and hybrid mesons in QCD, Nucl.Phys. A675 (2000), 54C-63C, Latex2e sources 10 pages. Plenary talk given at Hadrons 99 (Beijing) Report-no: Montpellier preprint PM/99-September.

[34] Philip R. Page, Eric S. Swanson, and Adam P. Szczepaniak, Hybrid meson decay phenomenology, Phys.Rev. D59 (1999), 034016.

[35] F. Von Hippel and C. Quigg, Centrifugal-barrier effects in resonance partial decay widths, shapes, and production amplitudes, Phys.Rev. D5 (1972), 624638.

[36] A. Zaitsev, Study of exotic resonances in diffractive reactions, Nucl.Phys. A675 (2000), 155C-160C.

[37] Benedikt Zihlmann, Tof detector description for cdr, GlueX-doc-994-v4 (2008). 